\title{
Marine Controlled-Source Electromagnetic Interferometry
}





\section{Marine Controlled-Source Electromagnetic Interferometry}

\section{PROEFSCHRIFT}

ter verkrijging van de graad van doctor aan de Technische Universiteit Delft,

op gezag van de Rector Magnificus prof.ir. K.C.A.M. Luyben, voorzitter van het College voor Promoties,

in het openbaar te verdedigen op maandag 21 mei 2012 om 10.00 uur

door Jürg Werner HUNZIKER

Master of Science ETH

(Eidgenössische Technische Hochschule Zürich, Zwitserland)

geboren te Luzern, Zwitserland. 
Dit proefschrift is goedgekeurd door de promotoren:

Prof.dr.ir. E.C. Slob

Prof.dr.ir. C.P.A. Wapenaar

Samenstelling promotiecommissie:

Rector Magnificus,

Prof.dr.ir. E.C. Slob,

Prof.dr.ir. C.P.A. Wapenaar,

Prof.dr. W.A. Mulder,

Prof.dr. J. Bruining,

Dr. W. Walk,

Dr. J. Singer,

Prof.dr.ir. J. van der Kruk,
Technische Universiteit Delft, voorzitter

Technische Universiteit Delft, promotor

Technische Universiteit Delft, promotor

Technische Universiteit Delft

Technische Universiteit Delft

Shell

Fugro

RWTH Aachen University

This work is financially supported by the Dutch Technology Foundation (STW), the Applied Science division of the Netherlands Organization for Scientific Research (NWO), and the Technology Program of the Ministry of Economic Affairs.

ISBN 978-90-8570-995-4

(C) 2012 by J.W. Hunziker. Some rights reserved. Chapters 2 is adapted from published work (C) 2011 SEG, DOI: 10.1190/1.3587222) and reprinted here with permission from SEG. Chapter 3 is adapted from published work (C) 2011 EAGE, DOI: 10.1111/j.1365-2478.2011.01019.x) and reprinted here with permission from EAGE. Chapter 5 is based on published work (C) 2011 SEG, DOI: 10.1190/1.3589112 and (C) IEEE) and reprinted here with permission from SEG and IEEE.

Printed in the Netherlands by: CPI Wöhrmann Print Service, Zutphen 


\section{Contents}

1 Introduction 1

2 Effects of the airwave in time-domain marine CSEM 5

2.1 Introduction . . . . . . . . . . . . . . 6

2.2 Method ...................... 7

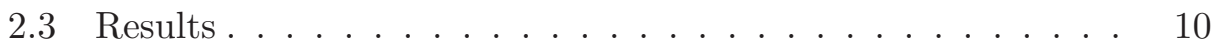

2.3.1 Point source . . . . . . . . . . . . . 10

2.3.2 Elongated source . . . . . . . . . . . . . . 14

2.3.3 Effects of the airwave .............. . 15

2.4 Conclusions . . . . . . . . . . . . . . 18

2.5 Appendix A: Coefficients, functions, and parameters of equation 2.1 . . . . . . . . . . . . . . . . . . . . . . . 19

2.6 Appendix B: Approximations of the airwave term . . . . . 23

3 2D CSEM Interferometry by MDD: spatial sampling aspects 25

3.1 Introduction . . . . . . . . . . . . 26

3.2 Theory................... 30

3.3 Acquisition Aspects . . . . . . . . . . . . . . 35

3.4 Processing . . . . . . . . . . . . . . . . . 39

3.5 Results: Physical Source . . . . . . . . . . . . . . . 41

3.6 Stability analysis . . . . . . . . . . . . . . 45

3.7 Results: Synthetic Aperture Source . . . . . . . . . . . . . . . 47

3.8 Noise Analysis . . . . . . . . . . . . . . . . 52

3.9 Conclusions ................... . . . . 54

3.10 Appendix A: The matrices $\tilde{\Gamma}, \tilde{\mathbf{L}}$ and $\tilde{\mathbf{L}}^{-1} \ldots \ldots . . . . . \quad$. 56

4 EM interferometry in 3D in wavenumber and space domains 59

4.1 Introduction . . . . . . . . . . . . . . . 60

4.2 Theory................... . . 62

4.3 Model ...................... 65 
4.4 Synthesized-2D interferometry in the frequency-wavenumber domain ................... . . 66

4.5 Synthesized-2D interferometry in the frequency-space domain 72

4.6 Full-3D interferometry in the frequency-wavenumber domain 74

4.7 Full-3D interferometry in the frequency-space domain . . . . 80

4.8 Reservoir detectability . . . . . . . . . . . . . . 83

4.9 Parameter dependency . . . . . . . . . . . . . . 85

4.10 Discussion ................... . . . 87

4.11 Conclusions . . . . . . . . . . . . . . . 88

4.12 Appendix A: The 2D decomposition algorithm . . . . . . . 90

5 Time-lapse CSEM using Interferometry 91

5.1 Introduction . . . . . . . . . . . . . . . . 91

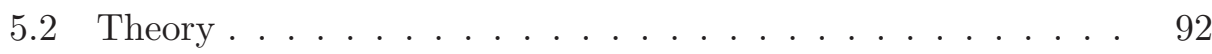

5.3 Results..................... . . 93

5.3.1 Scenario 1................ . . 95

5.3.2 Scenario 2................. . . 95

5.3.3 Scenario 3 ................ 97

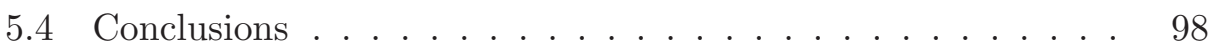

6 Conclusions and outlook 101

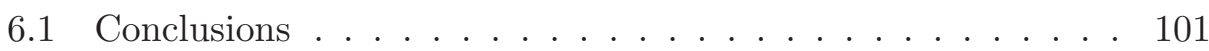

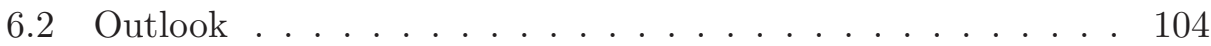

$\begin{array}{ll}\text { Bibliography } & 105\end{array}$

$\begin{array}{ll}\text { Summary } & 113\end{array}$

$\begin{array}{ll}\text { Samenvatting } & 115\end{array}$

$\begin{array}{lr}\text { Acknowledgements } & 119\end{array}$

$\begin{array}{ll}\text { Curriculum Vitae } & 121\end{array}$ 


\section{Chapter 1}

\section{Introduction}

In the quest to find new oil and gas reservoirs, companies mainly rely on seismic exploration methods. Reflection seismics is able to produce a highresolution structural image of the subsurface (e.g., Yilmaz, 1987) and amplitude variation with offset analysis techniques are able to estimate parameters of potential reservoirs (e.g., Sickle \& Valusek, 1990; Castagna \& Swan, 1997; Ursenbach \& Stewart, 2008). But seismic approaches are not suited to distinguish the nature of the fluid content in the rock (Wright et al., 2002; Wilt \& Alumbaugh, 1998). Since water-saturated reservoirs exhibit a low electric resistivity and hydrocarbon-saturated reservoirs a high electric resistivity (Wright et al., 2002; Hesthammer et al., 2010), electromagnetic methods may be able to add the key information in order to determine the pore-fluid of a potential reservoir (Um \& Alumbaugh, 2007).

Out of four exploration wells drilled above a potential reservoir, on average one produces oil commercially. Thus, the wildcat commercial success rate of the oil industry lies at approximately 25\% (Hesthammer et al., 2010). Using a database of 86 wells for which electromagnetic data was available, the study by Hesthammer et al. (2010) investigates the usefulness of the information from electromagnetic surveys. Among the hydrocarbon bearing wells in their study, $70 \%$ showed an electromagnetic anomaly after a very simple processing scheme. A study by Johansen et al. (2008) investigated technical instead of commercial success rates. They consider a survey as successful if the indication of the presence or absence of a reservoir due to an electromagnetic survey after a sophisticated processing scheme is consistent with reality. They found, that in $85 \%$ of all the cases, the conclusions drawn from an electromagnetic survey were correct. The studies of Hesthammer et al. (2010) and Johansen et al. (2008) indicate the value of electromagnetic methods to the oil and gas industry and explain the necessity to develop 
these methods to a reliable tool.

In this thesis, the focus lies on the marine Controlled-Source Electromagnetic (CSEM) method. In this technique, the electromagnetic field generated by a controlled source is recorded with a sparse set of stationary multicomponent receivers at the ocean bottom. Typically, the source, a horizontal electric-field transmitter, is towed by a boat close to the sea floor to maximize the energy that couples to sea floor rocks (Constable \& Srnka, 2007). The source antenna is 50 to $300 \mathrm{~m}$ long and emits a signal at frequencies between $0.1 \mathrm{~Hz}$ and $10 \mathrm{~Hz}$ (Eidesmo et al., 2002; Constable, 2010). In frequency-domain CSEM, which is the method considered in this thesis, only the fundamental frequency of the monochromatic source signal and sometimes higher order harmonics are considered for interpretation (MacGregor et al., 2006). In contrast, time-domain CSEM uses a source signal with many frequencies in order to retrieve the impulse response of the subsurface (Ziolkowski, 2007).

There are various processing and interpretation approaches with different degrees of sophistication. The simplest approach is to normalize the data with a reference receiver, which is located in an area where no hydrocarbons are expected. If the amplitude of the resulting normalized magnitude-versus-offset plot is significantly larger than one, the presence of a subsurface resistor, possibly a hydrocarbon reservoir, is indicated (e.g., Dell'Aversana, 2007). So-called source-receiver range bins allow to plot these normalized amplitudes on maps in case of a larger dataset (Ellingsrud et al., 2002). A more sophisticated processing approach is to apply an inversion for the subsurface conductivity distribution (for an overview see Avdeev, 2005). The inversion can be based on CSEM data only (e.g., Zhdanov \& Hursan, 2000; Plessix \& Mulder, 2008), include structural information from seismics (e.g., MacGregor \& Sinha, 2000; Harris et al., 2009; Key, 2009) or jointly invert seismic and CSEM data (e.g., Hu et al., 2009).

Problems related to processing and interpretation of CSEM data are: Firstly, the signal does not only interact with the subsurface on its path from the source to the receiver. On the contrary, a large part of the signal diffuses directly from the source to the receiver through the water or via the air-water interface. This signal does not contain any information about the subsurface, instead it can mask the subsurface response making an interpretation of the data difficult. Secondly, the location of the source is only known with limited precision. In an inversion scheme for the subsurface conductivity distribution or in time-lapse measurements, precise knowledge of the source location is crucial.

In this thesis, the capabilities and limitations of interferometry by mul- 
tidimensional deconvolution (MDD), as an intermediate processing step for marine CSEM data to overcome these problems, are investigated. With interferometry by MDD, any interactions of the signal with the air-water interface and the water itself can be suppressed. Furthermore, the source is redatumed to any receiver location to overcome source uncertainties. The idea is to apply interferometry before the inversion for the subsurface conductivity distribution. Suppressing the interactions of the signal with the air-water interface and reducing source uncertainties should lead to a better defined solution space.

This thesis begins with an introductory chapter (chapter 2) explaining and visualizing the diffusion of an electric field. Although the focus of this thesis lies on frequency-domain CSEM, in this chapter the electric fields are displayed in the time domain. The time domain allows to retrieve the directionality of the electric field in the sense of a temporal and spatial expanding high-amplitude area, with the highest amplitude remaining at the source location. This leads to an understanding why for example beam steering of diffusive fields and decomposition in upward and downward decaying diffusive fields are possible. The latter is a crucial part for interferometry by MDD as will be discussed in the successive chapters. The representation of the electric field in time and space also gives insights into the airwave, a signal that interacts with the air-water interface. In chapter 2, the properties of the airwave and its appearance as a function of antenna orientation are discussed. While chapter 2 is devoted to a large part to discuss the airwave, the following chapters concentrate on interferometry by MDD in order to remove the airwave from CSEM data.

After chapter 2, the reader is prepared for the core of this thesis, which is formed by chapter 3. This chapter features an extensive discussion on interferometry, CSEM and the benefits that can be gained from combining the two. After the theoretical background is explained, this chapter focuses on the required dense receiver sampling for CSEM-interferometry and how this problem can be solved by using the synthetic aperture source concept. Finally, the effect of noise, receiver orientation errors, as well as receiver positioning errors on the method are illustrated.

While the treatment in chapter 3 has been entirely in $2 \mathrm{D}$, chapter 4 deals with synthesized-2D and full-3D CSEM-interferometry. Synthesized-2D interferometry uses 2D data that were created from 3D data by integration over a set of source lines off the receiver line, assuming no or only little variations of the medium in the crossline direction. This process simulates an infinitely long source in the crossline direction and allows therefore 2D-processing. The optimal source-line distribution in order to create sufficiently accurate syn- 
thesized 2D data while minimizing the amount of source lines is discussed. In contrast, full-3D interferometry requires recordings on an areal grid of receivers. The introduced algorithms allow to retrieve the full-3D reflection response for the transverse-magnetic-mode, the transverse-electric-mode as well as mode conversions. Synthesized-2D as well as full-3D interferometry algorithms for the frequency-wavenumber and the frequency-space domains are presented. The effect of an inaccurate conductivity value for the decomposition and improperly chosen stabilization parameter for the least-squares inversion are investigated as well.

Finally, the advantage of CSEM-interferometry for time-lapse CSEM is demonstrated in chapter 5 . Since interferometry redatums the source to a receiver location, the source repeatability problem can be solved. Numerical examples for two production methods, homogeneous sweep and bottom flooding, are presented. Interferometry by MDD is applied to mitigate effects of source-position inaccuracy and changes in ocean conductivity.

The thesis is rounded off in chapter 6 with conclusions and suggestions for further research. 


\title{
Chapter 2
}

\section{Effects of the airwave in time-domain marine controlled-source electromagnetics ${ }^{\mathrm{i}}$}

\begin{abstract}
In marine time-domain controlled-source electromagnetics (CSEM), there are two different acquisition methods: with horizontal sources for fast and simple data acquisition or with vertical sources for minimizing the effects of the airwave. Illustrations of the electric field as a function of space and time for various source antenna orientations, based on analytical formulation of the electric field in two half-spaces, provide insights into the properties of the airwave and the nature of diffuse electric fields. Observing the development of the electric field over time and space reveals that diffusive fields exhibit directionality. Therefore, techniques that have thus far mostly been applied to wavefields can be adapted for CSEM. Examples range from the well-known up-down decomposition to beam steering. Vertical sources have the advantage of not creating an airwave. On the other hand, it is quite difficult to achieve perfect verticality of the source antenna. Results, using a numerically modelled data set to analyse the impact of the airwave on a signal from a subsurface reservoir in the case of a slightly dipping vertical source, indicate that already for a dip of $0.05^{\circ}$, the airwave contributes $20 \%$ to the complete electric field in our configuration of reservoir depth, water thickness, and conductivity values.
\end{abstract}

${ }^{\mathrm{i}}$ This chapter has been published as a journal paper in Geophysics, 76(4), F251-F261 (Hunziker et al., 2011a). Note that minor changes have been introduced to make the text consistent with the other chapters of this thesis. 


\section{$2.1 \quad$ Introduction}

Controlled-source electromagnetics (CSEM) in a marine environment can be used to detect resistors, e.g., hydrocarbon reservoirs, in the subsurface. A historical overview of the method is given by Constable (2010). However, the recorded signal can be strongly affected by the airwave, which consists of energy refracted at the air-water interface, travelling through the air at the speed of light and continuously transmitting energy downward into the water. Except for geometric spreading, the airwave signal is not attenuated while travelling in the air (Wait, 1959; Bannister, 1984; King et al., 1992; Amundsen et al., 2006). Thus, the strength of the airwave depends mainly on its vertical diffusion path in the water (Um \& Alumbaugh, 2007). Consequently, if the source and receiver antennas are located far from the air-water interface, the airwave is weak.

Because the airwave produces a strong signal that masks the response from a subsurface resistor, one wants to eliminate it from the data during processing. In shallow water, it is possible that energy first interacts with the air-water interface to form an airwave and then interacts with the reservoir. Such an airwave signal contains information about a possible subsurface resistor (Mittet, 2008), but this information content is difficult to extract and interpret. For frequency-domain CSEM, different approaches exist to minimize the effects of the airwave from the data. These approaches include modelling and adjacent subtraction (Nordskag \& Amundsen, 2007), weighted differences between pairs of receivers or sources (Løseth et al., 2010; Chen \& Alumbaugh, 2011), taking the frequency derivative (Maaø \& Nguyen, 2010; Chen \& Alumbaugh, 2011) or a difference between fields at two frequencies that are further apart (Wirianto et al., 2011), magnetotelluric impedance stripping (Chen \& Alumbaugh, 2011), wavefield decomposition (Amundsen et al., 2006), and interferometry (Wapenaar et al., 2008b).

Weiss (2007) shows that the CSEM time-domain response from a subsurface resistor in shallow water may be easier to distinguish from the airwave arrival than in the frequency domain because of the possible separation of the two events in time. In the case of overlapping events, the airwave can be removed by filtering (Ziolkowski \& Wright, 2007). Some airwave-removal methods for frequency-domain CSEM may also be applicable for time-domain CSEM.

In this chapter, we investigate the effects of the airwave for time-domain CSEM. Two different offshore CSEM methods are available in the time domain. The first uses horizontal sources and receivers (Chave et al., 1991; Ziolkowski, 2007). The processing scheme proposed by Ziolkowski (2007) de- 
convolves the recorded signal with the source signal, a pseudorandom binary sequence, to recover the earth impulse response. In contrast, the second method, described by Holten et al. (2009), uses vertical sources and receivers. In this method, the source signal consists of eight square pulses followed by silent periods. The signal is stacked over these eight pulses to reduce noise.

The advantage of using vertical sources and receivers is the complete absence of the airwave. Additionally, by using numerical models, Constable \& Weiss (2006) show that, in contrast to CSEM with horizontal receivers, vertical receivers are more sensitive to the edges of a subsurface resistor. Consequently, measurements of the vertical electric field are more suitable for mapping the lateral extent of a resistor than recordings of the horizontal electric field.

Unfortunately, acquisition with vertical sources and receivers also has disadvantages. First, a vertical source cannot be dragged through the water behind a boat. This reduces the acquisition speed dramatically. Second, because of currents, seabed topography, and other effects, it can be quite difficult to manage a perfect vertical orientation of sources and receivers. And third, the amplitude of the signal is lower compared to CSEM with horizontal sources and receivers (Holten et al., 2009).

The goals of this chapter are to illustrate the appearance and the properties of the airwave in marine CSEM and to investigate the effects of imperfect verticality of the antennas when using vertical sources. To analyse the properties of the airwave, we model the electric field analytically in a half-space of water below a half-space of air. Then we use a numerically modelled electric field in a more complicated model with a finite-length reservoir in the subsurface to investigate the effects of imperfect verticality of the source antenna.

\subsection{Method}

We analytically model the electric field in a homogeneous half-space of water (conductivity $\sigma=3 \mathrm{~S} / \mathrm{m}$ ) below a half-space of air $(\sigma=0 \mathrm{~S} / \mathrm{m})$. The source is located $150 \mathrm{~m}$ below the air-water interface. The setup of the analytical model is summarized in Figure 2.1a.

Raiche \& Coggon (1975) give the expressions for the electric field in the lower half-space in the space-frequency domain. Here, we use a spacetimedomain expression given by Slob et al. (2010):

$$
E_{k r}=P_{k r}+\sum_{m=0}^{2}\left(g_{k r ; m}^{t e ;-} F_{m}^{-}+g_{k r ; m}^{t e ;+} F_{m}^{+}+g_{k r ; m}^{t m ;-} F_{m}^{-}+g_{k r ; m}^{t m ;+} F_{m}^{+}\right),
$$


a)

$\vdash--\underline{8000 \mathrm{~m}}----1$

air: $\sigma=0 \mathrm{~S} / \mathrm{m}$

water: $\sigma=3 \mathrm{~S} / \mathrm{m} \quad 0 \mathrm{~m}$

b)

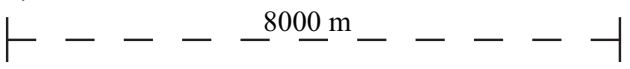

air: $\sigma=0 \mathrm{~S} / \mathrm{m}$

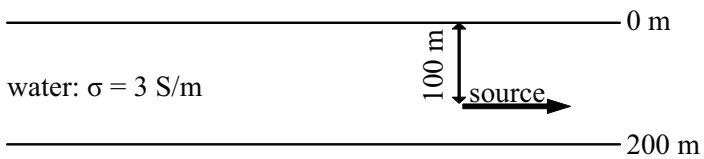

ground: $\sigma=0.5 \mathrm{~S} / \mathrm{m}$

$$
\frac{\mid \text { reservoir: } \sigma=0.02 \mathrm{~s} / \mathrm{m}}{\vdash-\frac{4}{4000} \overline{\mathrm{m}}-1}-600 \mathrm{~m}
$$

Figure 2.1: Modelling setups for the (a) analytical and (b) numerical models (not to scale).

where $k$ indicates the receiver orientation and $r$ indicates the source orientation $x, y$, and $z$ (or inline, crossline, and vertical directions, respectively). There are five terms on the right-hand side of Equation 2.1. The first term $P_{k r}$ describes the effects of the airwave in the subsurface and contains modified Bessel functions of integer order. The other four terms consist of a coefficient $g_{k r ; m}$ multiplied with an exponential time-dependent diffusion function $F_{m}^{ \pm}$. The first part of the superscripts TE or TM of the coefficient indicates if the corresponding term describes the transverse electric (TE) mode part or the transverse magnetic (TM) mode part of the electric field, respectively. The minus or plus sign in the second part of the superscript indicates the direct and reflected (via the interface) travel paths, respectively.

In other words, the electric field consists of five parts: the airwave effect, the direct and reflected TE-mode parts, and the direct and reflected TMmode parts. If either the source or the receiver or both are vertical, the coefficients for the TE mode are zero because in that case there is no TE mode. Furthermore, $P_{k r}$ is also zero, which means that no airwave exists for 
these source/receiver combinations. All coefficients and diffusion functions are derived in Slob et al. (2010) for a vertical transverse isotropic (VTI) medium and are summarized and simplified for an isotropic half-space in Appendix A.

In Appendix B (section 2.6), we give an approximation for the Bessel functions in the airwave term $P_{k r}$ for early times and one for late times. The combination of both approximations in the appropriate time window results in a normalized error no larger than $4 \%$. However, in all of our computations, we use the expression for the airwave, including the Bessel functions and thus without approximations.

Each of the three source orientations - $x, y$, and $z$ - is modelled for all three receiver components for different depths, horizontal offsets, and time steps. As a result of reciprocity, $E_{x y}$ and $E_{y x}$ are identical. Fields $E_{x z}$ and $E_{z x}$ as well as $E_{y z}$ and $E_{z y}$ differ from each other only in the sign of the reflected field (Raiche \& Coggon, 1975; Slob et al., 2010). Although the current dipole source is modelled as a point, i.e., no physical length, different antenna orientations can be simulated by modelling just one component of the source. In the following, a vertical source actually means a vertically oriented point-dipole source. The source-time function is a Dirac delta function.

To investigate the interference of the airwave with a subsurface response, depending on the dip of a vertical source, a slightly more complicated setup is modelled. This setup consist of three layers, in which the last contains a reservoir with finite extent. The geometry and electrical parameters are shown in Figure 2.1b. The electric field in this setup is modelled numerically using a frequency-domain multigrid code (Mulder, 2006). Modelling is done for several frequencies, followed by an interpolation and a fast Fourier transform (Mulder et al., 2008).

The data cubes are combined geometrically to simulate new source-antenna orientations for both modelling procedures. The orientation of an antenna is defined using two angles: angle $\alpha$ describes dips of the antenna relative to the vertical coordinate axis $z$ and angle $\beta$ describes the azimuth relative to the horizontal coordinate axis $x$ (Figure 2.2). For horizontal, inline-directed receivers, the data cubes can be combined to form any source-antenna orientation using the following formulation:

$$
E_{x s}=E_{x x} \cos (\beta) \sin (\alpha)+E_{x y} \sin (\beta) \sin (\alpha)+E_{x z} \cos (\alpha),
$$

where the subscript $s$ in $E_{x s}$ indicates any arbitrary source-antenna orientation. The fields $E_{x x}, E_{x y}$, and $E_{x z}$ are inline-component (x-direction) electric fields from a unit source antenna in the $x-, y$-, and $z$-directions, respectively. The same is done for the crossline (y-direction) and the vertical receiver com- 
ponent (z-direction) of the electric field, which are then added up to the total electric field using

$$
E_{t s}=\sqrt{E_{x s}^{2}+E_{y s}^{2}+E_{z s}^{2}}
$$

where the subscript $t$ represents the total electric field on the receiver side. With this procedure, any source orientation can be created and used to analyse the impact of the airwave on the data as a function of the source-antenna orientation.

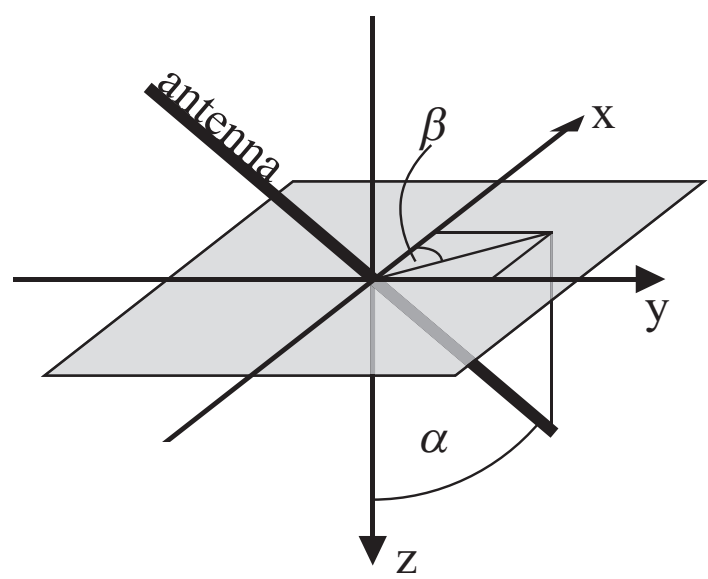

Figure 2.2: Relation between angles $\alpha$ (dip) and $\beta$ (azimuth).

\subsection{Results}

\subsubsection{Point source}

Figure 2.3 shows the development of the electric field over time in a half-space of water (see Figure 2.1a) after a vertical source $\left(\alpha=\beta=0^{\circ}\right)$ at a depth of $150 \mathrm{~m}$ is excited. Five time slices between $10^{-3} \mathrm{~s}$ and $10^{1} \mathrm{~s}$ are displayed. The source is modelled as a point-dipole source in space and time (Dirac delta function). The left column contains vertical cross sections as a function of depth and inline offset, whereas the right column contains horizontal cross sections as a function of inline and crossline offset. The colour represents the amplitude of the total electric field, and the arrows indicate the orientation of the field within the plane displayed. Note that the boundary between the two half-spaces is at a depth of $0 \mathrm{~m}$. Only the lower half-space, filled with water, is shown. 

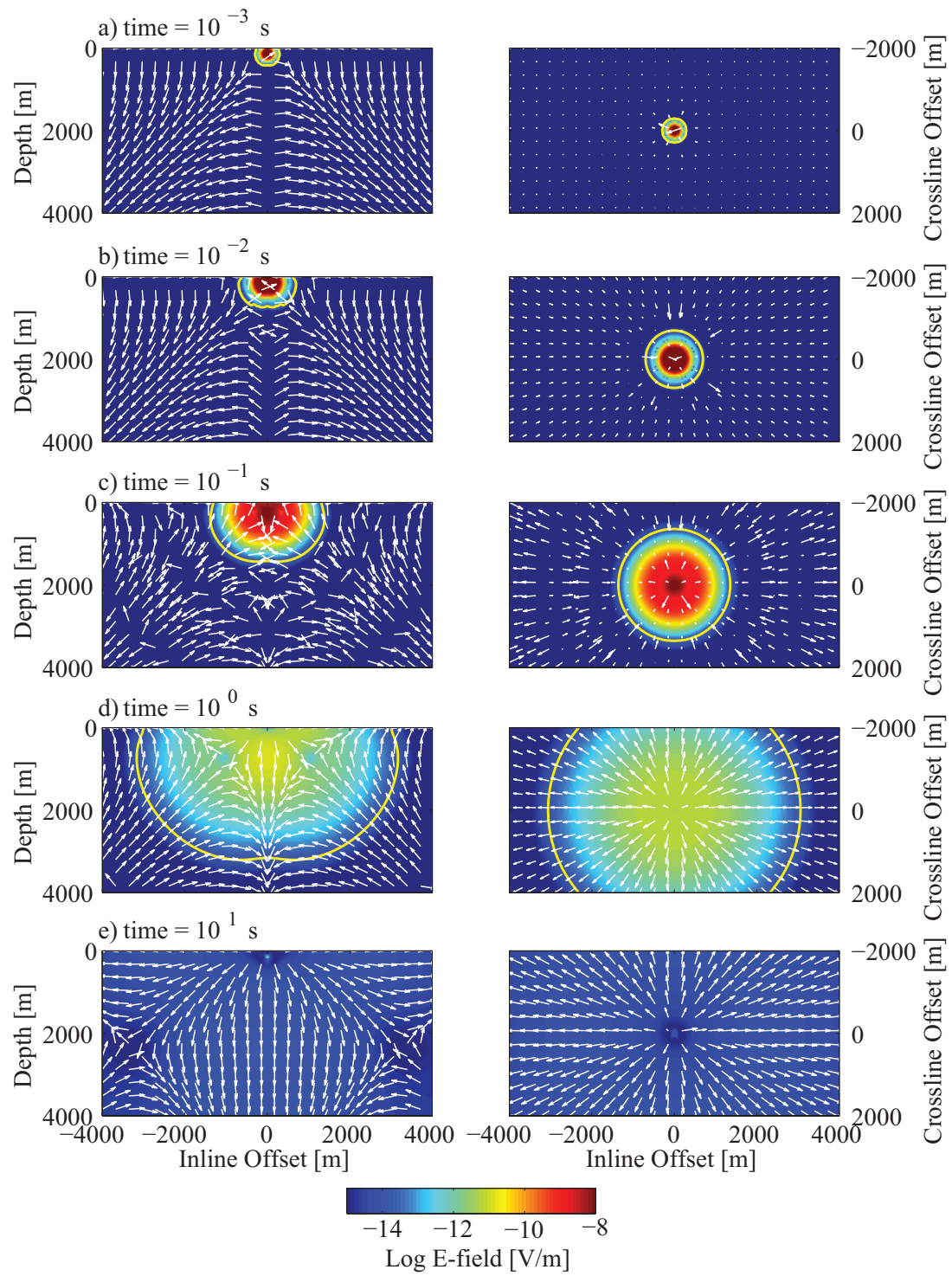

Figure 2.3: The diffusion of the electric field resulting from a vertical electric source in a halfspace of water $(\sigma=3 \mathrm{~S} / \mathrm{m})$ below a half-space of air $(\sigma=0 \mathrm{~S} / \mathrm{m})$ for different time steps: (a) $10^{-3} \mathrm{~s}$, (b) $10^{-2} \mathrm{~s}$, (c) $10^{-1} \mathrm{~s}$, (d) $10^{0} \mathrm{~s}$, and (e) $10^{1} \mathrm{~s}$ after the excitation of the source. The left column shows a vertical cross section in the inline direction at a crossline offset of $0 \mathrm{~m}$. The right column shows a horizontal cross section at a depth of $200 \mathrm{~m}$. The colour stands for the amplitude of the total electric field between $10^{-15} \mathrm{~V} / \mathrm{m}$ (blue) and $10^{-8} \mathrm{~V} / \mathrm{m}$ (red). The arrows represent the orientation of the field within the plane shown. The yellow line indicates an amplitude of the electric field of $10^{-14} \mathrm{~V} / \mathrm{m}$. 
In the time slices, the diffusion of the electric field away from the source location can be seen as a high-amplitude (red) area that is expanding over time. We use the term diffusion front to name the zone where the amplitude of the electric field switches in one time step from undetectable to detectable in the next time step. This diffusion front, a function of space and time, is emphasized by the yellow curve, which highlights positions where the electric field has a strength of $10^{-14} \mathrm{~V} / \mathrm{m}$. Although in CSEM the electric field does not behave like a wave but like a diffusive field, it exhibits directionality, as indicated by the propagation of the diffusion front. This directionality is not related with the directionality from the vector nature of the electric field (white arrows). In contrast to a wavefield, the largest amplitude always remains at the source location. This is also the case for other source-time functions, such as the switch-on function (Heaviside step function) or the derivative of the Dirac delta function (Doublet function).

The electric field consists of a direct field and a reflected field, but no refracted energy - i.e., no airwave - is visible (Um \& Alumbaugh, 2007) because a vertical source generates a TM-mode field only. The TE mode, which creates the airwave, is not present; therefore, the airwave is absent. In other words, an electric field created by a vertical source can be fully described by the last two terms of equation 2.1. Precisely at the boundary, the vertical component of the electric field is zero, resulting in a perfectly horizontal electric-field vector at this location. The reason is the boundary condition, which demands that the vertical component of the electric current density $J=\sigma E$ be continuous across the interface (Nabighian, 1988). Because $J$ is zero in air, the vertical component of $J$ must be zero in the water at the boundary as well. Complete destructive interference between the vertical component of the direct and the reflected fields, from a TM-mode reflection coefficient of one, fulfils this boundary condition.

Figure 2.4 shows the diffusion of the electric field for the same setup but for a horizontally oriented source in the inline direction $\left(\alpha=90^{\circ}\right.$ and $\left.\beta=0^{\circ}\right)$. In this situation, the total field consists of the TE and TM modes; therefore, the airwave is expected to appear. The amplitude of the airwave starts to exceed $10^{-15} \mathrm{~V} / \mathrm{m}$ at the air-water interface at $10^{-2.45} \mathrm{~s}$ after the excitation of the source. It can be identified as an event diffusing downward almost simultaneously at all offsets. The horizontal decay, visible at shallow depth, is caused by the geometric spreading term proportional to the inverse horizontal distance cubed. In Figure 2.4b and 2.4c, showing the electric field at later times, the airwave forms the major contribution to the electric field at large offsets and shallow depth. The electric-field vector is perfectly horizontal in this area because the airwave is a pure TE mode and therefore 
has no vertical electric component. This goes hand in hand with the previous observation that a vertical source, which produces a pure TM-mode field, does not feature the airwave. The airwave contribution has a late-time asymptote proportional to $t^{-3}$ but the direct field is proportional to $t^{-2.5}$.
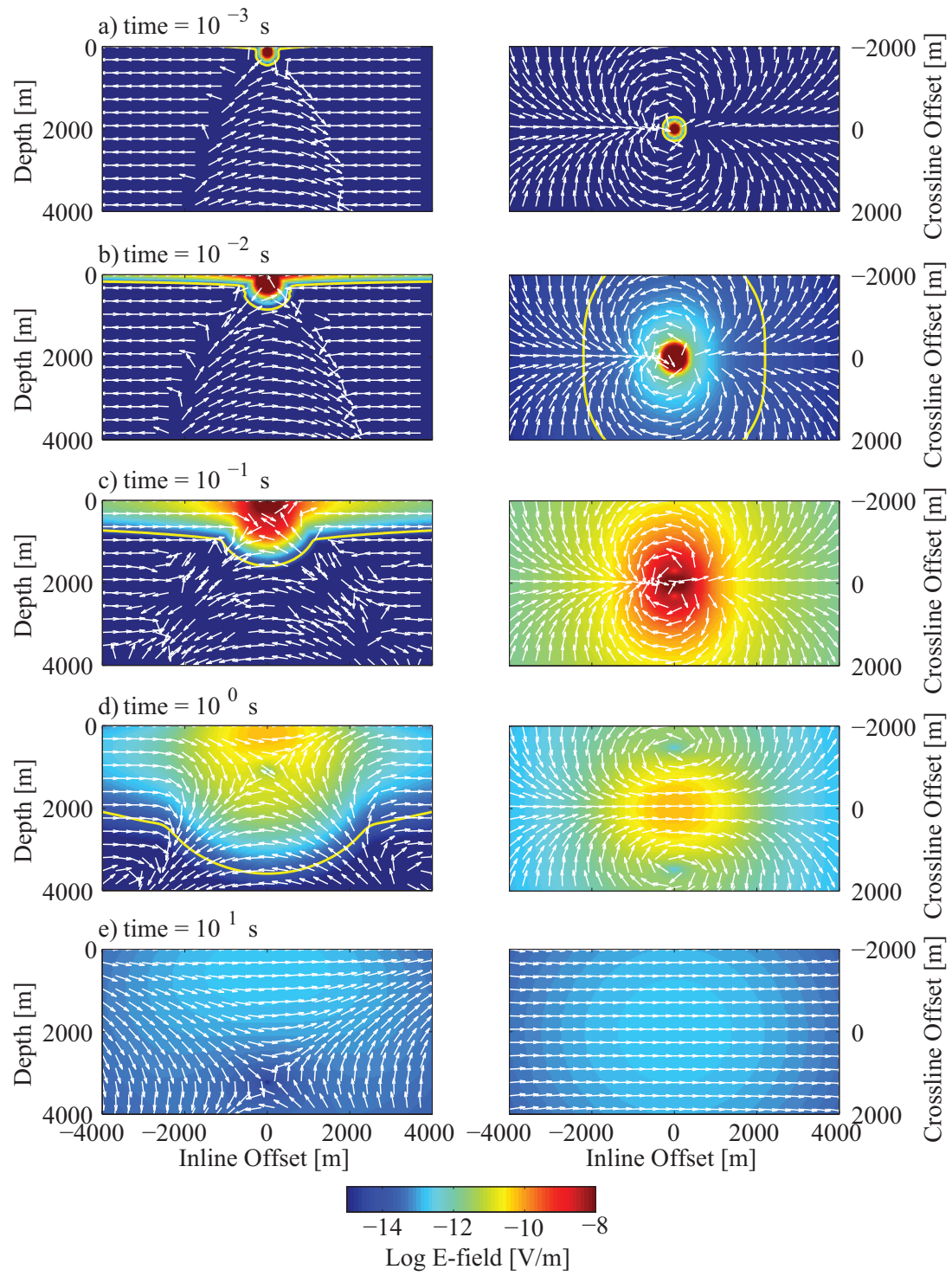

Figure 2.4: Same as Figure 2.3 but for a horizontal source. 


\subsubsection{Elongated source}

In reality, the source antenna is not a point but has a physical extension. To check how our modelling results change with an elongated source, a 200$\mathrm{m}$-long source antenna was simulated by integration over the length of the antenna. Because of the large computational cost of such an integration, this was only done for a few time steps. Figure 2.5 shows the electric field in the half-space of water for one of these time steps, which is $10^{-0.43} \mathrm{~s}$ after source excitation. The centre of the source is located at a depth of $150 \mathrm{~m}$, and the antenna is dipping $20^{\circ}$ in the inline direction $\left(\alpha=20^{\circ}\right.$ and $\left.\beta=0^{\circ}\right)$.
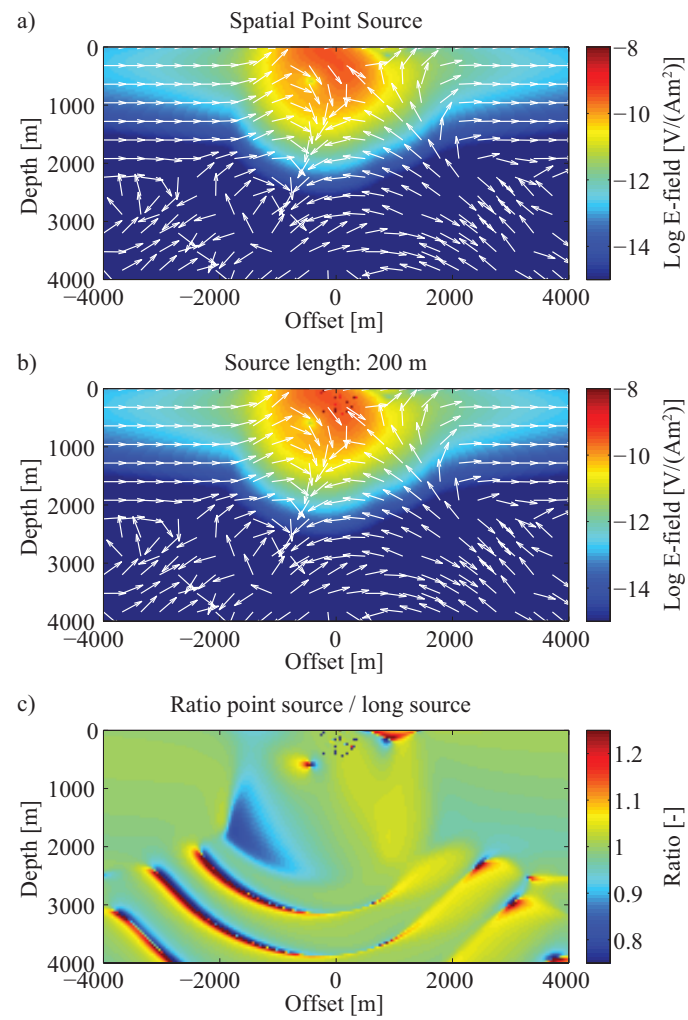

Figure 2.5: (a) Vertical cross section at a crossline offset of $0 \mathrm{~m}$, showing the electric field normalized by its source-dipole moment in the half-space of water $10^{-0.43}$ $s$ after source excitation. The spatial-point source is located at a depth of $150 \mathrm{~m}$, dipping $20^{\circ}$ in the inline direction. The colour represents the amplitude of the total electric field normalized by the dipole moment of the source on a common logarithmic scale $\left[V /\left(A m^{2}\right)\right]$. The arrows indicate the orientation of the field within the plane shown. (b) Same as (a), but the source antenna is $200 \mathrm{~m}$ long with its centre at a depth of $150 \mathrm{~m}$. (c) The ratio of (a) point source to (b) long source on a linear scale. 
In Figure 2.5a, the electric field resulting from a point source is shown as in the previous figures; in Figure 2.5b, the source is $200 \mathrm{~m}$ long and the electric field has been normalized with the source-dipole moment to make the strength of the electric field independent of the antenna length. Because of the integration, some artefacts around the source location appear. Figure $2.5 \mathrm{c}$ shows the ratio of the electric field resulting from the point source to that from the elongated source of $200 \mathrm{~m}$. This ratio is approximately one almost everywhere except at kinks where the electric field changes direction abruptly. Consequently, the observations made for a point source are also valid for an elongated source because an elongated-source response is similar to a point-source response if the resulting field is normalized by the sourcedipole moment.

\subsubsection{Effects of the airwave}

In this section, we investigate the effects of the airwave and its interference with the subsurface response, which includes the scattered field from the reservoir as well as the response from the ocean bottom for the case of an imperfect vertical source. Therefore, we numerically model the electric field as a function of time and dip angle of the source for the situation plotted in Figure 2.1b at three points. These three points are located at the ocean bottom at a depth of $200 \mathrm{~m}$ and at inline offsets of 500, 1000, and 2000 $\mathrm{m}$. The source antenna dips between $0^{\circ}$ - a perfect vertical source and $1^{\circ}$. Assuming a length of $200 \mathrm{~m}$ for the vertical source antenna, a dip of $1^{\circ}$ means that the top point of the antenna is mispositioned by $3.5 \mathrm{~m}$.

Figure 2.6a-2.6c shows only the airwave at these three points. The airwave appears at all offsets at $10^{-2} \mathrm{~s}$ after the excitation of the source. Because the arrival time of the airwave depends only on the vertical distances between the source and the air-water interface and between the receiver and the air-water interface but not on the horizontal offset, the arrival time is the same for all three sample points. As a result of the geometric spreading of the electric field in the air, the amplitude of the airwave is offset-dependent. Only for perfect verticality of the source does the amplitude of the airwave remain below the detection level. As soon as the source is dipped slightly, the airwave appears with a significant amplitude.

Figure 2.6d-2.6f shows the airwave, the reflected field at the air-water interface, and the direct field in the water layer. Although the airwave is dependent on the dip angle of the source antenna, the direct and reflected fields seem to be invariant to the angles under consideration. With increasing offset, the contribution from the airwave separates in time from the rest of the 

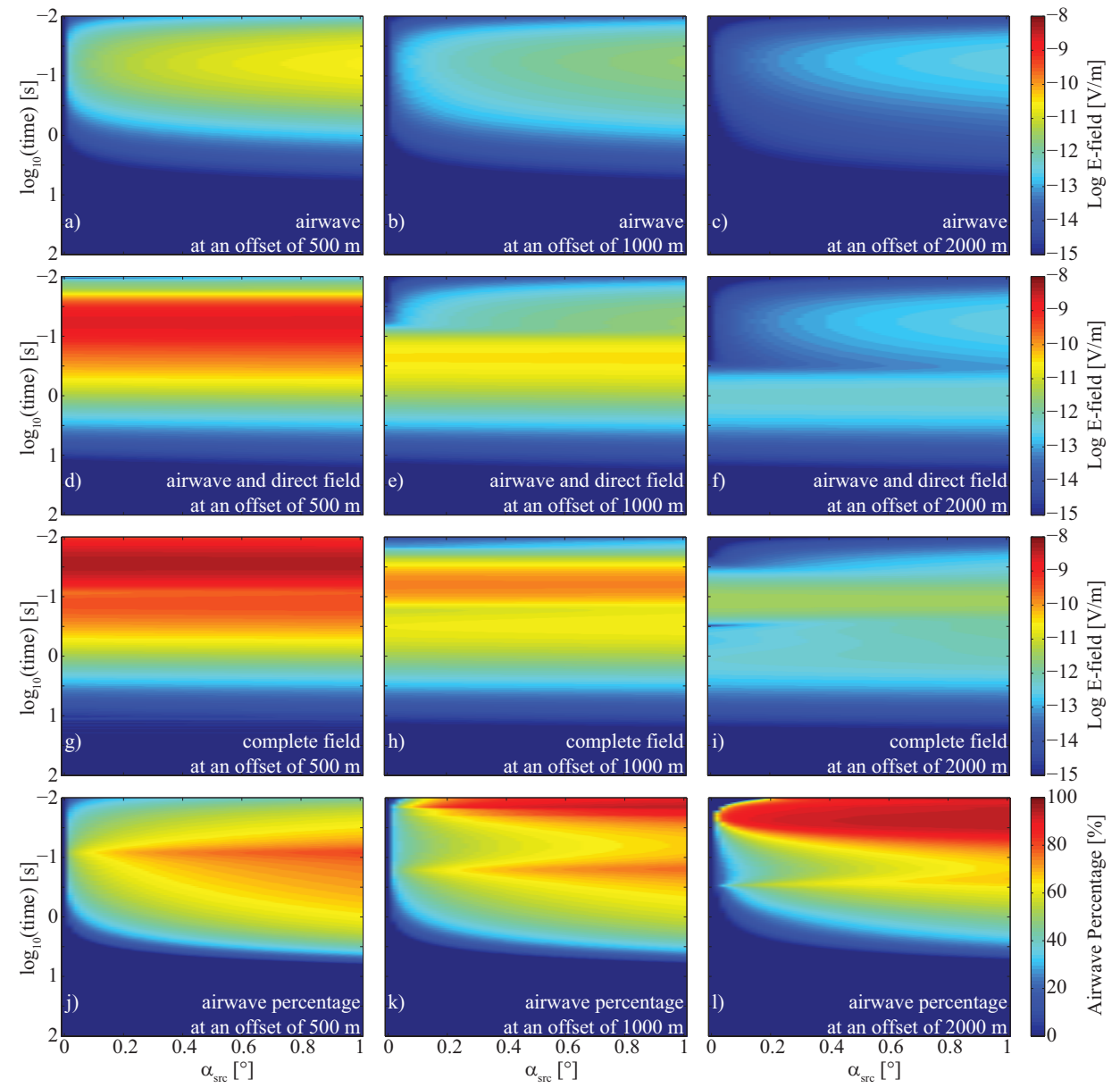

Figure 2.6: The electric field as a function of time and dip angle of the source for three points at a depth of $200 \mathrm{~m}$ and inline offsets of 500, 1000, and $2000 \mathrm{~m}$. (Top row) Airwave only at offsets of (a) $500 \mathrm{~m}$, (b) $1000 \mathrm{~m}$, and (c) $2000 \mathrm{~m}$. (Second row) The airwave, reflected field, and direct field in the water at offsets of (d) 500 m, (e) $1000 \mathrm{~m}$, and (f) $2000 \mathrm{~m}$. (g-i) Complete field, i.e., all events, including the response from the reservoir. ( $j-l)$ Percentage of airwave relative to the complete field. In (a-i), the colour represents the amplitude of the total electric field on a common logarithmic scale. In $(j-l)$, the colour represents the percentage of the airwave on a linear scale. 
field. The separation in time is possible because, on the one hand, the direct and reflected fields appear at later times with increasing offset, whereas the onset of the airwave remains invariant in time and, on the other hand, the airwave decays faster in time than the direct and reflected fields (Slob et al., 2010).

The complete field - the airwave, the reflected field, the direct field, and the subsurface response (i.e., the reservoir response and the response from the ocean bottom) - is shown in Figure 2.6g-2.6i. Similar to the direct field, the subsurface response is invariant to the angles under consideration. In the subsurface, especially in the reservoir, the diffusion front moves much faster than in the water layer because of the smaller conductivity. As a result of this and the shallow location of the reservoir, the subsurface response appears earlier than the direct field, leading to an overlap of the subsurface response with the airwave for our modelling setup (Figure 2.1b). In other words, the airwave hides the signal from the reservoir in our choice of reservoir depth and conductivity values. A better separation in time between the airwave and the reservoir response may be possible for a deeper reservoir.

The percentage of the airwave relative to the complete field, i.e., the field that contains all events, is shown in Figure 2.6j-2.61. The contribution of the airwave at an offset of $2000 \mathrm{~m}$ for early times is almost $100 \%$ because it is basically the only component of the electric field at that moment. The direct and reflected fields appear later in time and are weaker in amplitude with increasing offset, so the airwave becomes more dominant at these large offsets. There is no airwave only for a perfect vertical source. As soon as there is a dip in the source, there is a contribution of the airwave to the complete electric field.

It is difficult to conclude how large of a percentage of airwave signal can be coped with, but it is possible to quantify the dip angle for a given airwave percentage. In other words, it is possible to plot the dip angle as a function of airwave percentage for specified offsets and time steps. These graphs are shown in Figure 2.7 for offsets of 500, 1000, and $2000 \mathrm{~m}$. These dip-angleversus-airwave-percentage curves were computed for four different time steps at each offset location. All curves have the shape of an exponential function. This means that a large increase of airwave contribution happens for small dips of the source antenna. Because of the limited number of computed source dip angles, this steep increase appears as a step at $0 \%$ airwave contribution in the graph. If the source antenna already dips strongly, i.e., more than $50^{\circ}$ an additional dip causes little change in the airwave percentage. To ensure that the airwave is always smaller than $20 \%$ at all offsets, the source antenna should not be allowed to dip more than $0.05^{\circ}$. For a 200-m-long antenna, 
this corresponds to a positioning error of less than $0.2 \mathrm{~m}$.
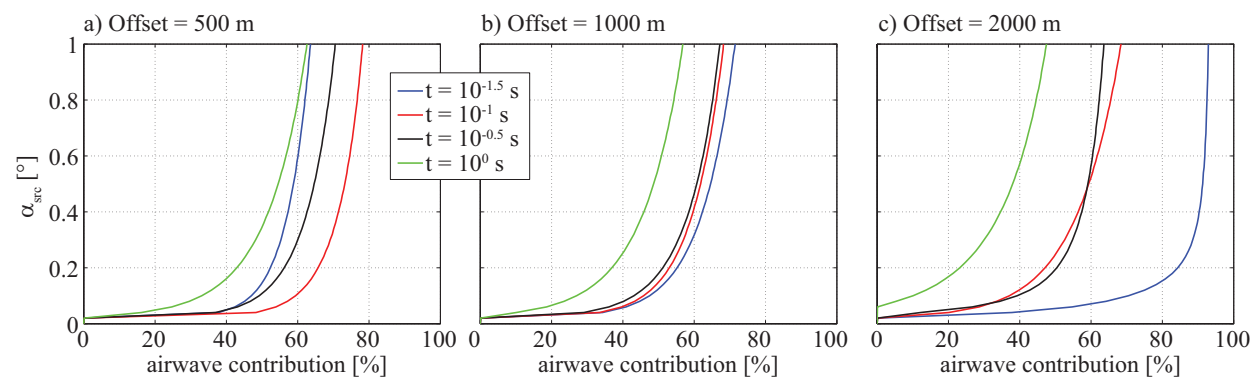

Figure 2.7: Source dip angle $\alpha_{\text {src }}$ as a function of the airwave percentage for four different time steps and three receiver locations: (a) $500 \mathrm{~m}$, (b) $1000 \mathrm{~m}$, and (c) $2000 \mathrm{~m}$.

\subsection{Conclusions}

The directionality of wavefields is widely accepted, but the directionality of diffusive fields is often questioned. The development of the diffusive electromagnetic fields in space and time shows that diffusive fields exhibit directionality in the sense of a temporal and spatial expanding high-amplitude area, with the highest amplitude remaining at the source location. Consequently, if a diffusive field has a directionality comparable to a wavefield, techniques that thus far have been applied mostly to wavefields can be adapted for CSEM. Examples are decomposition into upward- and downward-decaying fields, decomposition and deconvolution of upward- by downward-decaying fields to obtain the CSEM impulse reflection response, and beam steering.

The amplitude of the electric field resulting from an elongated-source antenna is comparable with the amplitude of the electric field from a pointsource antenna if normalized with the source-dipole moment, which is a constant factor. In other words, the amplitude difference does besides at kinks of the electric field in most regions not exceed 10\%. Consequently, observations made for a point source are also valid for an elongated source.

Vertical receivers are insensitive to the airwave, and a vertical source does not excite the airwave in a horizontally layered medium because the airwave has only horizontal components. Unfortunately, small deviations of the source antenna from verticality lead to a significant contribution of the airwave to the complete measured field, e.g., a dip of $0.05^{\circ}$ results in a contribution of the airwave of $20 \%$ with respect to the complete electric field, including the subsurface response. Still, for a horizontal source, the contri- 
Table 2.1: Parameters used in Appendix A (section 2.5).

\begin{tabular}{|lc|}
\hline description & expression \\
\hline \hline conductivity & $\sigma$ \\
free space value of magnetic permeability & $\mu_{0}$ \\
Laplace transformation parameter & $s$ \\
squared vertical wavenumber of the lower halfspace & $\gamma=\sqrt{s \mu_{0} \sigma}$ \\
receiver depth, source depth & $x_{3}, x_{3}^{S}$ \\
vertical distance from source to receiver & $h^{ \pm}=\left|x_{3}^{ \pm} x_{3}^{S}\right|$ \\
horizontal distance in space & $\rho=\sqrt{x_{1}^{2}+x_{2}^{2}}$ \\
radial distance from source to receiver & $r^{ \pm}=\sqrt{\rho^{2}+\left(h^{ \pm}\right)^{2}}$ \\
time & $t$ \\
offset dependent diffusion time & $\tau^{ \pm}=\frac{\sigma \mu_{0}\left(r^{ \pm}\right)^{2}}{4}$ \\
\hline
\end{tabular}

bution of the airwave would be much larger. In our setup, the subsurface response appears at similar times as the airwave. The latter is thus masking a possible signal from a reservoir with a dipping source antenna. In that case, the airwave needs to be suppressed or separated in time-domain CSEM using similar methods as applied in frequency-domain CSEM. An airwave-removal scheme will usually result in fewer artifacts if the amplitude of the airwave is already relatively small. Consequently, it can still be advantageous to have an antenna dip slightly from verticality than a horizontally oriented antenna. For up/down separation or interferometry, multicomponent receivers are necessary.

\subsection{Appendix A: Coefficients, functions, and para- meters of equation 2.1}

The following equations are published for a VTI medium in Slob et al. (2010) and are shown here for the isotropic case. In this section, we use subscript notation. The coordinate vector $\mathbf{x}$ is therefore $\left(x_{1}, x_{2}, x_{3}\right)^{T}$ where the superscript $T$ stands for transposition. Table 2.1 lists all parameters required in the following coefficients and functions.

The TM-mode coefficients for the horizontal components are given by

$$
g_{\alpha \beta ; 0}^{T M ; \pm}=\frac{3 x_{\alpha} x_{\beta}-\left(r^{ \pm}\right)^{2} \delta_{\alpha \beta}}{4 \pi \sigma\left(r^{ \pm}\right)^{5}},
$$




$$
\begin{aligned}
& g_{\alpha \beta ; 1}^{T M ; \pm}=\left(\frac{3 x_{\alpha} x_{\beta}-\left(r^{ \pm}\right)^{2} \delta_{\alpha \beta}}{4 \pi\left(r^{ \pm}\right)^{4}}-\frac{2 x_{\alpha} x_{\beta}-\rho^{2} \delta_{\alpha \beta}}{4 \pi \rho^{4}}\right) \frac{\sqrt{\mu_{0}}}{\sqrt{\sigma}}, \\
& g_{\alpha \beta ; 2}^{T M ; \pm}=\frac{\mu_{0} x_{\alpha} x_{\beta}}{4 \pi r^{ \pm}}\left(\frac{1}{\left(r^{ \pm}\right)^{2}}-\frac{1}{\rho^{2}}\right),
\end{aligned}
$$

where the subscripts $\alpha$ and $\beta$ can have the values one or two, representing the horizontal components. The Kronecker delta $\delta_{\alpha \beta}$ is one for $\alpha=\beta$ and zero otherwise. The TE-mode coefficients for the horizontal components are given by

$$
\begin{aligned}
& g_{\alpha \beta ; 0}^{T E ;-}=0 \\
& g_{\alpha \beta ; 0}^{T E ;+}=\frac{3 f_{\alpha \beta}-\left(r^{+}\right)^{2} \delta_{\alpha \beta}}{2 \pi \sigma\left(r^{+}\right)^{5}}, \\
& g_{\alpha \beta ; 1}^{T E ;-}=\frac{x_{\alpha} x_{\beta}-f_{\alpha \beta}}{4 \pi \rho^{4}} \frac{\sqrt{\mu_{0}}}{\sqrt{\sigma}}, \\
& g_{\alpha \beta ; 1}^{T E ;+}=\left(\frac{3 f_{\alpha \beta}-\left(r^{+}\right)^{2} \delta_{\alpha \beta}}{2 \pi \sqrt{\sigma}\left(r^{+}\right)^{4}}+\frac{x_{\alpha} x_{\beta}-f_{\alpha \beta}}{4 \pi \rho^{4}}\right) \frac{\sqrt{\mu_{0}}}{\sqrt{\sigma}}, \\
& g_{\alpha \beta ; 2}^{T E ;-}=-\frac{\mu_{0} f_{\alpha \beta}}{4 \pi \rho^{2} r^{-}}, \\
& g_{\alpha \beta ; 2}^{T E ;+}=\frac{\mu_{0} f_{\alpha \beta}}{4 \pi r^{+}}\left(\frac{2}{\left(r^{+}\right)^{2}}-\frac{1}{\rho^{2}}\right),
\end{aligned}
$$

where $f_{\alpha \beta}=\left(\rho^{2} \delta_{\alpha \beta}-x_{\alpha} x_{\beta}\right)$. For the vertical components, only TM-mode coefficients are non-zero:

$$
\begin{aligned}
g_{3 \alpha ; 0}^{T M ;-} & =\frac{3 x_{\alpha}\left(x_{3}-x_{3}^{S}\right)}{4 \pi \sigma\left(r^{-}\right)^{5}}, \\
g_{3 \alpha ; 0}^{T M ;+} & =\frac{3 x_{\alpha} h^{+}}{4 \pi \sigma\left(r^{+}\right)^{5}}, \\
g_{3 \alpha ; 1}^{T M ;-} & =\frac{3 x_{\alpha}\left(x_{3}-x_{3}^{S}\right)}{4 \pi\left(r^{-}\right)^{4}} \frac{\sqrt{\mu_{0}}}{\sqrt{\sigma}}, \\
g_{3 \alpha ; 1}^{T M ;+} & =\frac{3 x_{\alpha} h^{+}}{4 \pi\left(r^{+}\right)^{4}} \frac{\sqrt{\mu_{0}}}{\sqrt{\sigma}} \\
g_{3 \alpha ; 2}^{T M ;-} & =\mu_{0} \frac{x_{\alpha}\left(x_{3}-x_{3}^{S}\right)}{4 \pi\left(r^{-}\right)^{3}},
\end{aligned}
$$




$$
g_{3 \alpha ; 2}^{T M ;+}=\mu_{0} \frac{x_{\alpha} h^{+}}{4 \pi\left(r^{+}\right)^{3}} .
$$

In view of reciprocity,

$$
\begin{aligned}
& g_{\alpha 3 ; m}^{T M ;-}=g_{3 \alpha ; m}^{T M ;-}, \\
& g_{\alpha 3 ; m}^{T M ;+}=-g_{3 \alpha ; m}^{T M ;+} .
\end{aligned}
$$

The last coefficients are found as

$$
\begin{aligned}
& g_{33 ; 0}^{T M ; \pm}=\mp \frac{\left(3\left(h^{ \pm}\right)^{2}-\left(r^{ \pm}\right)^{2}\right)}{4 \pi \sigma\left(r^{ \pm}\right)^{5}}, \\
& g_{33 ; 1}^{T M ; \pm}=\mp \frac{\left(3\left(h^{ \pm}\right)^{2}-\left(r^{ \pm}\right)^{2}\right)}{4 \pi\left(r^{ \pm}\right)^{4}} \frac{\sqrt{\mu_{0}}}{\sqrt{\sigma}}, \\
& g_{33 ; 2}^{T M ; \pm}=\mp \mu_{0} \frac{\left(\left(h^{ \pm}\right)^{2}-\left(r^{ \pm}\right)^{2}\right)}{4 \pi\left(r^{ \pm}\right)^{3}} .
\end{aligned}
$$

The diffusion functions are given by

$$
\begin{aligned}
& F_{m}^{-}=F_{m}\left(t, \tau^{-}\right), \\
& F_{m}^{+}=F_{m}\left(t, \tau^{+}\right),
\end{aligned}
$$

where

$$
\begin{aligned}
& F_{0}(t, \tau)=\sqrt{\frac{\tau}{\pi t^{3}}} \exp (-\tau / t) H(t), \\
& F_{1}(t, \tau)=\left(\frac{\tau}{t}-\frac{1}{2}\right) \sqrt{\frac{1}{\pi t^{3}}} \exp (-\tau / t) H(t), \\
& F_{2}(t, \tau)=\left(\frac{\tau}{t}-\frac{3}{2}\right) \sqrt{\frac{\tau}{\pi t^{5}}} \exp (-\tau / t) H(t) .
\end{aligned}
$$

Here, $H(t)$ denotes the unit step function. The horizontal components of the term $P_{k r}$ are given by

$$
\begin{aligned}
P_{\alpha \beta}= & \frac{\sigma \mu_{0}^{2} h^{+} \exp \left(-\tau_{h}^{+} / t\right)}{32 \pi t^{3}}\left\{2\left(\delta_{\alpha \beta}-\frac{x_{\alpha} x_{\beta}}{\rho^{2}}\right) \bar{I}_{1}\left(\tau_{\rho} / t\right)\right. \\
& \left.-\left(\frac{\sigma \mu_{0}}{2 t}\left(\rho^{2} \delta_{\alpha \beta}-x_{\alpha} x_{\beta}\right)-\delta_{\alpha \beta}\right)\left(\bar{I}_{0}\left(\tau_{\rho} / t\right)-\bar{I}_{1}\left(\tau_{\rho} / t\right)\right)\right\},
\end{aligned}
$$


where $\tau_{h}^{+}=\sigma \mu_{0}\left(h^{+}\right)^{2} / 4$ and $\tau_{\rho}=\sigma \mu_{0} \rho^{2} / 8$. The scaled modified Bessel functions $\bar{I}_{0}$ and $\bar{I}_{1}$ are related to the modified Bessel functions $I_{0}$ and $I_{1}$ as follows:

$$
\bar{I}_{n}\left(\tau_{\rho} / t\right)=\exp \left(-\tau_{\rho} / t\right) I_{n}\left(\tau_{\rho} / t\right) .
$$

All other airwave terms, i.e., $P_{3 \alpha}, P_{\alpha 3}$ and $P_{33}$, are zero.

In equation 2.1, we use coefficients for the TE and TM modes. In an isotropic medium, the diffusion functions are the same for both modes, so equation 2.1 can be simplified to

$$
E_{k r}=P_{k r}+\sum_{m=0}^{2}\left(g_{k r ; m}^{-} F_{m}^{-}+g_{k r ; m}^{+} F_{m}^{+}\right),
$$

with the coefficients

$$
\begin{aligned}
& g_{k r ; 0}^{-}=\frac{3\left(x_{k}-x_{k}^{S}\right)\left(x_{r}-x_{r}^{S}\right)-\left(r^{-}\right)^{2} \delta_{k r}}{4 \pi \sigma\left(r^{-}\right)^{5}}, \\
& g_{k r ; 1}^{-}=\frac{3\left(x_{k}-x_{k}^{S}\right)\left(x_{r}-x_{r}^{S}\right)-\left(r^{-}\right)^{2} \delta_{k r}}{4 \pi\left(r^{-}\right)^{4}} \frac{\sqrt{\mu_{0}}}{\sqrt{\sigma}}, \\
& g_{k r ; 2}^{-}=\frac{\left(x_{k}-x_{k}^{S}\right)\left(x_{r}-x_{r}^{S}\right)-\left(r^{-}\right)^{2} \delta_{k r}}{4 \pi\left(r^{-}\right)^{3}} \mu_{0}, \\
& g_{\alpha \beta ; 0}^{+}=-3 \frac{x_{\alpha} x_{\beta}+\left(\left(r^{+}\right)^{2}-2 \rho^{2}\right) \delta_{\alpha \beta}}{4 \pi \sigma\left(r^{+}\right)^{5}}, \\
& g_{\alpha \beta ; 1}^{+}=-3 \frac{x_{\alpha} x_{\beta}+\left(\left(r^{+}\right)^{2}-2 \rho^{2}\right) \delta_{\alpha \beta}}{4 \pi\left(r^{+}\right)^{4}} \frac{\sqrt{\mu_{0}}}{\sqrt{\sigma}}, \\
& g_{\alpha \beta ; 2}^{+}=-\frac{x_{\alpha} x_{\beta}+\left(\left(r^{+}\right)^{2}-2 \rho^{2}\right) \delta_{\alpha \beta}}{4 \pi\left(r^{+}\right)^{3}} \mu, \\
& g_{k 3 ; 0}^{+}=-\frac{3\left(x_{k}+x_{k}^{S}\right)\left(x_{3}+x_{3}^{S}\right)-\left(r^{+}\right)^{2} \delta_{k 3}}{4 \pi \sigma\left(r^{+}\right)^{5}}, \\
& g_{k 3 ; 1}^{+}=-\frac{3\left(x_{k}+x_{k}^{S}\right)\left(x_{3}+x_{3}^{S}\right)-\left(r^{+}\right)^{2} \delta_{k 3}}{4 \pi\left(r^{+}\right)^{4}} \frac{\sqrt{\mu_{0}}}{\sqrt{\sigma}}, \\
& g_{k 3 ; 2}^{+}=-\frac{\left(x_{k}+x_{k}^{S}\right)\left(x_{3}+x_{3}^{S}\right)-\left(r^{+}\right)^{2} \delta_{k 3}}{4 \pi\left(r^{+}\right)^{3}}, \\
& g_{3 \beta ; 0}^{+}=\frac{3 x_{\beta}\left(x_{3}+x_{3}^{S}\right)}{4 \pi \sigma\left(r^{+}\right)^{5}},
\end{aligned}
$$




$$
\begin{aligned}
& g_{3 \beta ; 1}^{+}=\frac{3 x_{\beta}\left(x_{3}+x_{3}^{S}\right)}{4 \pi\left(r^{+}\right)^{4}} \frac{\sqrt{\mu_{0}}}{\sqrt{\sigma}}, \\
& g_{3 \beta ; 2}^{+}=\frac{x_{\beta}\left(x_{3}+x_{3}^{S}\right)}{4 \pi\left(r^{+}\right)^{3}} \mu_{0} .
\end{aligned}
$$

From this, we arrive at the delta function response:

$$
\begin{aligned}
E_{k r} & =P_{k r} \\
& +\left(\frac{\sigma \mu_{0}}{4 \pi t}\right)^{\frac{3}{2}}\left(\frac{\delta_{k r}}{\sigma t}+\frac{\left(x_{k}-x_{k}^{S}\right)\left(x_{r}-x_{r}^{S}\right)-\left(r^{-}\right)^{2} \delta_{k r}}{4 t^{2}} \mu_{0}\right) \exp \left(-\frac{\tau^{-}}{t}\right) \\
& -\left(\frac{\sigma \mu_{0}}{4 \pi t}\right)^{\frac{3}{2}} \delta_{k \alpha} \delta_{r \beta}\left(\frac{x_{\alpha} x_{\beta}+\left(\left(r^{+}\right)^{2}-2 \rho^{2}\right) \delta_{\alpha \beta}}{4 t^{2}}\right) \mu_{0} \exp \left(-\frac{\tau^{+}}{t}\right) \\
& -\left(\frac{\sigma \mu_{0}}{4 \pi t}\right)^{\frac{3}{2}} \delta_{r 3}\left(\frac{\delta_{k 3}}{\sigma t}+\frac{\left(x_{k}+x_{k}^{S}\right)\left(x_{3}+x_{3}^{S}\right)-\left(r^{+}\right)^{2} \delta_{k 3}}{4 t^{2}} \mu_{0}\right) \exp \left(-\frac{\tau^{+}}{t}\right) \\
& +\left(\frac{\sigma \mu_{0}}{4 \pi t}\right)^{\frac{3}{2}} \delta_{r \beta} \delta_{k 3} \frac{x_{\beta}\left(x_{3}+x_{3}^{S}\right)}{4 t^{2}} \mu_{0} \exp \left(-\frac{\tau^{+}}{t}\right) .
\end{aligned}
$$

\subsection{Appendix B: Approximations of the airwave term}

Although we use the complete expression for the airwave in our computation, approximations are possible. For early times, i.e., $t \ll \tau_{\rho}$ or in other words for large arguments, the Bessel functions $\bar{I}_{n}$ in equation 2.29 can be approximated by (Abramowitz \& Stegun, 1972)

$$
\begin{aligned}
& \bar{I}_{0}\left(\tau_{\rho} / t\right) \approx \frac{1}{\sqrt{2 \pi \frac{\tau_{\rho}}{t}}}\left\{1+\frac{t}{8 \tau_{\rho}}+\frac{9}{2}\left(\frac{t}{8 \tau_{\rho}}\right)^{2}+\frac{75}{2}\left(\frac{t}{8 \tau_{\rho}}\right)^{3}\right\}, \\
& \bar{I}_{1}\left(\tau_{\rho} / t\right) \approx \frac{1}{\sqrt{2 \pi \frac{\tau_{\rho}}{t}}}\left\{1-3 \frac{t}{8 \tau_{\rho}}-\frac{15}{2}\left(\frac{t}{8 \tau_{\rho}}\right)^{2}-\frac{105}{2}\left(\frac{t}{8 \tau_{\rho}}\right)^{3}\right\} .
\end{aligned}
$$

Figure 2.8a shows the normalized error for the airwave term $P_{11}$ using equations 2.45 and 2.46 to replace the Bessel functions $\bar{I}_{n}$ in equation 2.29. The relative error is plotted for seven different horizontal distances $\rho$.

For late times, i.e., $t \gg \tau_{\rho}$ (for small arguments), the Bessel functions $\bar{I}_{n}$ can be approximated as (Abramowitz \& Stegun, 1972)

$$
\bar{I}_{0}\left(\tau_{\rho} / t\right) \approx \exp \left(-\frac{\tau_{\rho}}{t}\right)\left\{1+\left(\frac{\tau_{\rho}}{2 t}\right)^{2}+\frac{1}{4}\left(\frac{\tau_{\rho}}{2 t}\right)^{4}\right\},
$$



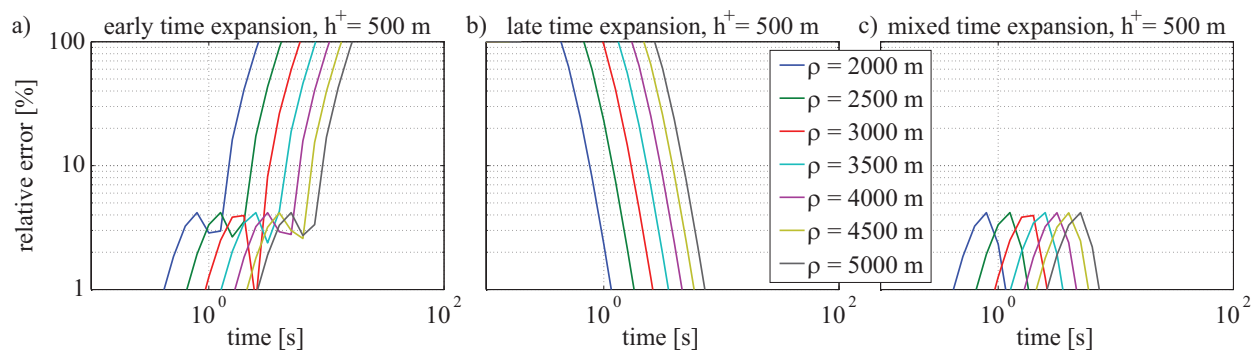

Figure 2.8: Relative error for the airwave term $P_{11}$ using (a) early (equations 2.45 and 2.46), (b) late (equations 2.47 and 2.48), and (c) mixed-time approximations for $h^{+}=500 \mathrm{~m}$.

$$
\bar{I}_{1}\left(\tau_{\rho} / t\right) \approx \exp \left(-\frac{\tau_{\rho}}{t}\right) \frac{\tau_{\rho}}{2 t}\left\{1+\frac{1}{2}\left(\frac{\tau_{\rho}}{2 t}\right)^{2}+\frac{1}{12}\left(\frac{\tau_{\rho}}{2 t}\right)^{4}\right\} .
$$

The relative error for the airwave term $P_{11}$ using equations 2.47 and 2.48 to replace the Bessel functions $\bar{I}_{n}$ in equation 2.29 is shown in Figure 2.8b. The two approximations are combined such that equations 2.45 and 2.46 are used if $t<\tau_{\rho} / 2$ and equations 2.47 and 2.48 if $t>\tau_{\rho} / 2$. The relative error for this combination is plotted in Figure 2.8c. At early and late times, the error is small, but it peaks as expected at intermediate times. The largest error is approximately $4 \%$. 


\title{
Chapter 3
}

\section{Two-dimensional Controlled-Source Electromagnetic Interferometry by multidimensional deconvolution: spatial sampling aspects ${ }^{\mathrm{i}}$}

\begin{abstract}
We use numerically modelled datasets to investigate the sensitivity of electromagnetic interferometry by multidimensional deconvolution to the spatial receiver sampling. Interferometry by multidimensional deconvolution retrieves the reflection response below the receivers after decomposition of the fields into upward and downward decaying fields and deconvolving the upward decaying field by the downward decaying field. Thereby the medium above the receiver level is replaced with a homogeneous half-space, the sources are redatumed to the receiver level, and the direct field is removed. Consequently, in a marine setting the retrieved reflection response is independent of any effect of the water layer and the air above. A drawback of interferometry by multidimensional deconvolution is the possibly unstable matrix inversion which is necessary to retrieve the reflection response. Additionally, in order to correctly separate the upward and the downward decaying fields, the electromagnetic fields need to be sampled properly. We show that the largest possible receiver spacing depends on two parameters: the vertical distance between the source and the receivers, and the length of the source.
\end{abstract}

${ }^{\mathrm{i}}$ This chapter has been published online and will be in print in 2012 as a journal paper in Geophysical Prospecting. Note that minor changes have been introduced to make the text consistent with the other chapters of this thesis. 
The receiver spacing should not exceed the larger of these two parameters. Besides these two parameters, the presence of inhomogeneities close to the receivers may also require a dense receiver sampling. We show that by using the synthetic aperture concept, an elongated source can be created from conventionally acquired data in order to overcome these strict sampling criteria. Finally, we show that interferometry may work under real-world conditions with random noise and receiver orientation and positioning errors.

\subsection{Introduction}

In geophysical exploration, seismic interferometry is known as the process of cross-correlating recordings at two receiver positions to retrieve the Green's function between these two receivers. The theory has been derived by various authors for lossless (Schuster et al., 2004; Wapenaar, 2004) and dissipative media (Snieder, 2006), and it has been applied in passive (Draganov et al., 2006) as well as in controlled-source seismics (Bakulin \& Calvert, 2006). A more complete overview on seismic interferometry can be found in Wapenaar et al. (2010a,b) or Schuster (2009). The concept of Green's function retrieval by cross-correlation has been extended also to electromagnetic waves (Slob et al., 2007a) and is called electromagnetic interferometry. For electromagnetic diffusive fields, interferometry by cross-correlation requires sources to be present throughout the whole volume of interest (Snieder, 2006), which is not practical. Therefore, another approach for interferometry with diffusive fields is needed.

It has been shown that the process of cross-correlation (CC) can be replaced by a multi-dimensional deconvolution (MDD) in both the controlledsource case (Wapenaar et al., 2008b) and the passive case (Wapenaar et al., 2008a). The advantages of MDD include elimination of the source signature, improved radiation characteristics of the retrieved source, and relaxation of the assumption of a lossless medium. Furthermore, interferometry by MDD works with one-sided illumination, whereas interferometry by $\mathrm{CC}$ requires a closed surface of sources. On the other hand, MDD is more expensive and the involved matrix inversion may be unstable. Furthermore, in most cases a decomposition of the measured fields into up- and downgoing fields is necessary, which requires acquisition with multi-component receivers on a sufficiently dense grid. Interferometry by MDD also requires an array or a network of sensors and can not be done with two receivers only, in contrast to interferometry by cross-correlation. A similar approach to eliminate the overburden from multicomponent seismic data was presented by Holvik \& Amundsen (2005). 
Controlled-Source Electromagnetic (CSEM) data are often collected in a marine environment for oil exploration. In this technique a horizontal electric-dipole source is towed behind a boat emitting a low-frequency electric field, which diffuses through the water and through the subsurface. The field that diffuses above the source in the sea decays in the upward direction, while the field that diffuses below the source in the sea decays in the downward direction. When the diffusing field experiences changes in the electric conductivity or magnetic permeability, the reflected diffusive field decays in the direction opposite to the corresponding incident diffusing field. This means that after some time at any level in the subsurface diffusive fields occur that decay in the upward direction and in the downward direction. The resulting EM-field is recorded at the ocean bottom by horizontal multicomponent receivers as a function of offset (Constable \& Srnka, 2007). Unfortunately, not only subsurface responses are recorded but also the direct field in the water and interactions of the electric field with the sea surface are registered.

One strong event is the so-called airwave. It consists of that part of the electromagnetic field which diffuses vertically upward from the source to the sea surface, continues in the air as a propagating wave with the speed of light along the sea surface, refracts continuously back into the sea as a vertically downward diffusing field, and eventually is recorded at the receivers (Um \& Alumbaugh, 2007). The field amplitude decays exponentially with distance in the sea and in the earth, whereas in the air the amplitude decays proportional to the inverse of distance to the power 3 (Wait, 1959; Bannister, 1984; King et al., 1992; Amundsen et al., 2006; Slob et al., 2010). The direct field contributions at the receiver decay therefore exponentially with offset, while the airwave contribution decays exponentially as a function of two-way depth from source to water surface and back to the receiver. Therefore, at horizontal source-receiver offsets that are small compared to the two-way source-receiver depth, the recorded field is dominated by the direct field and reflections from the sea surface. On the other hand, in shallow seas and at relatively large offsets, the airwave is strong (Amundsen et al., 2006).

Since the airwave does not contain any information about the subsurface, one aims to minimize the effect of the airwave. Various approaches like modelling and adjacent subtraction (Nordskag \& Amundsen, 2007), weighted differences between pairs of receivers or sources (Chen \& Alumbaugh, 2011; Løseth et al., 2010), taking the frequency derivative (Chen \& Alumbaugh, 2011; Maaø \& Nguyen, 2010) or a difference between fields at two frequencies that are further apart (Wirianto et al., 2011), or magnetotelluric impedance stripping (Chen \& Alumbaugh, 2011) exist. Assuming that only the upward decaying field at the receiver level bears information about the subsurface 
and that the field which interacted with the air-water interface is downward decaying at the receiver level, Amundsen et al. (2006) proposed to decompose the diffusive fields into upward and downward decaying fields in order to remove the effects related to the air-water interface. They demonstrated this approach on computed data and on measured data. However, all interactions across the receiver level, e.g., the diffusive electric field interacting first with the air-water interface and subsequently with the reservoir, are not removed with this approach, which can be a problem in shallow water. Another approach to deal with the airwave is to carry out CSEM measurements in the time-domain instead of the frequency-domain, where the airwave is easier to separate from the subsurface response (Weiss, 2007; Andréis \& MacGregor, 2007).

Wapenaar et al. (2008b) and Nordskag et al. (2009) proposed to deconvolve the upward decaying field by the downward decaying field, both just below the receiver level. This can be applied to wave fields in dissipative media as well as to diffusive fields. This field decomposition followed by deconvolution was coined interferometry by multidimensional deconvolution (MDD) by Wapenaar et al. (2008b) and Lorentz water-layer elimination by Nordskag et al. (2009). We use here the term interferometry by MDD. The retrieved response is a response of the scattered field, where scattered field should be interpreted as the field that would exist if the medium above the receiver level were a homogeneous half-space and had the same properties as the medium just below the receiver level where the decomposition has been carried out. For this reason, all the interactions of the electromagnetic field with the air-water interface are removed from the recorded data, including the effects of the wave action of the surface sea waves. Consequently, also the in shallow-marine CSEM so problematic airwave is removed. This includes the above described interactions across the receiver level which are not removed with the approach of Amundsen et al. (2006).

Another advantage of MDD, besides the removal of the airwave, is that the locations and orientations of the actual sources do not need to be known. The imprint of the actual sources is removed from the data, and the receivers act as virtual sources. Consequently, it does not matter anymore if the source is towed close to the receivers at the ocean bottom or further above, which allows a more flexible survey design. In time-lapse CSEM for monitoring purposes, it is feasible to position the receivers at the same location in a second survey but it is almost impossible to position the source at the same location. This problem of repositioning the source at the same location can be circumvented by interferometry by MDD, which simply redatums the source from its unknown position to a receiver location. 
Hence, by applying interferometry by MDD to CSEM data, the source is redatumed to the receiver level, the direct field is eliminated, and the water layer is replaced by a homogeneous half-space that has the same properties as the medium directly below the receiver level (Wapenaar et al., 2008b). Note that interferometry by MDD is a data driven method. Only the material parameters just below the sea bottom, i.e., in most situations at an infinitesimal small step below the sea-sediment interface, are necessary for the decomposition. However, when there is a very thin layer below the receivers followed by a thicker layer, it can be advantageous to use the parameters of the thicker layer. In any case, no knowledge of the water layer is required. Drawbacks of this technique are the possibly unstable matrix inversion and the requirement for properly sampled data, i.e., regularly and dense enough sampled at all offsets. A numerical example of interferometry by MDD applied to CSEM data was given in Slob et al. (2007b), where all sea surface related effects were removed from the data resulting in a much clearer response of the target reservoir layer. A similar approach to remove the airwave from CSEM data is proposed by van den Berg et al. (2008) but in their algorithm the decomposition is carried out in the water layer. Consequently, the water layer is not removed, only the air-water interface is eliminated.

The retrieved reflection response can, for example, be used as a direct hydrocarbon indicator by normalizing it with a reflection response based on data acquired above a region without a reservoir. When a reservoir is present in one of the datasets, the resulting ratio is, for a laterally invariant reservoir, larger than one at all offsets. For standard CSEM data, this is only the case at intermediate offsets because at short offsets the data are dominated by the direct field and at large offsets by the airwave. A proper sampling is not necessary if the CSEM data are used as a direct hydrocarbon indicator in contrast to an inversion scheme that aims to retrieve the conductivity distribution of the subsurface from the data. In the latter case, proper sampling is necessary in order to prevent the inversion algorithm from getting unstable. Since interferometry by MDD requires proper sampling as well, we think interferometry is most useful as a preprocessing step for such an inversion algorithm. Most important is, that with interferometry by MDD applied, there is no need to model the water-layer and all its related effects in the forward step of the inversion process. Only the sub-bottom subsurface response needs to be modelled and, therefore, the solution space is expected to be better defined than using the recorded CSEM data in the inversion process directly. Furthermore, the forward problem can be solved much faster because the absence of the direct field allows to use a sparser computational grid. The source positions and orientations are not known precisely in 
the original data but they are known with a high precision after applying interferometry by MDD. This increases the accuracy of the inversion scheme further. Consequently, the retrieved reflection response can be used as a direct hydrocarbon indicator but is most useful in a scheme that inverts for the subsurface conductivity distribution.

The proposed methodology can be applied to CSEM data even when the unprocessed field data are not suitable as a direct hydrocarbon indicator. In that case, we can still retrieve subsurface information from the data using the MDD method. This may allow for retrieving the reservoir response, provided the data are sampled properly. The spatial bandwidth is primarily determined by the smallest distance between source and receivers, and by the size of sources and receivers. The reservoir response is smooth in the space domain and contains few high-wavenumber components because the reservoir is at a relatively large vertical distance from the receivers and the field is diffusive in nature, for which reason field components at large wavenumbers attenuate faster than those at small wavenumbers. For practical reasons, receiver stations need to be sparsely used, hence the vertical source-receiver distance and the source length are of interest because these can be chosen within practical limits.

In this chapter we investigate the required receiver spacing for a successful up-down decomposition and subsequent MDD using numerical examples for the conventional situation, where the source is vertically close to the receivers, and for the alternative situation, where the source is vertically far away from the receivers. We then present how synthetically elongated sources can be created in order to apply interferometry by MDD to datasets acquired with current acquisition techniques. Finally, noise issues are discussed to show the potential applicability of interferometry by MDD under real conditions.

\subsection{Theory}

Ampère's law and Faraday's law from Maxwell's equations are given in the space-frequency domain as

$$
\begin{aligned}
j \omega \hat{\varepsilon} \hat{E}_{i}+\hat{\sigma} \hat{E}_{i}-\epsilon_{i j k} \partial_{j} \hat{H}_{k} & =-\hat{J}_{i}^{e}, \\
j \omega \hat{\mu} \hat{H}_{k}+\epsilon_{k j i} \partial_{j} \hat{E}_{i} & =-\hat{J}_{k}^{m},
\end{aligned}
$$

where $\hat{E}_{i}=\hat{E}_{i}(\mathbf{x}, \omega)$ is the electric field strength, $\hat{H}_{k}=\hat{H}_{k}(\mathbf{x}, \omega)$ the magnetic field strength, and $\hat{J}_{i}^{e}=\hat{J}_{i}^{e}(\mathbf{x}, \omega)$ and $\hat{J}_{k}^{m}=\hat{J}_{k}^{m}(\mathbf{x}, \omega)$ are external electric and magnetic source functions, respectively. The vector $\mathbf{x}$ represents the three spatial coordinates. The material parameters electric permittivity, magnetic 
permeability, and conductivity are given by $\hat{\varepsilon}=\hat{\varepsilon}(\mathbf{x}, \omega), \hat{\mu}=\hat{\mu}(\mathbf{x}, \omega)$, and $\hat{\sigma}=\hat{\sigma}(\mathbf{x}, \omega)$, respectively, $\omega$ is the angular frequency and $j$ the imaginary unit. The circumflex denotes the space-frequency domain. The Levi-Civita tensor $\epsilon_{i j k}$ is equal to 1 for $\epsilon_{123}, \epsilon_{312}$ and $\epsilon_{231}$, equal to -1 for $\epsilon_{321}, \epsilon_{132}$ and $\epsilon_{213}$, and 0 otherwise. The spatial derivatives $\partial / \partial x_{j}$ are written in a short form as $\partial_{j}$. Latin subscripts can have the values 1, 2 or 3, representing the three spatial coordinates. Greek subscripts, on the other hand, can only have the values 1 or 2, representing the two horizontal coordinates. Einstein's summation convention is used for repeated subscripts.

Since we are dealing with diffusive fields, we neglect the term $j \omega \hat{\varepsilon} \hat{E}_{i}$ in equation (3.1). Next, the horizontal field components are separated from the vertical ones. At the receiver level, the resulting set of equations is combined such that the vertical components $\hat{E}_{3}$ and $\hat{H}_{3}$ are eliminated. This leads to the following matrix equation:

$$
\partial_{3} \hat{\mathbf{Q}}=\hat{\mathbf{A}} \hat{\mathbf{Q}}+\hat{\mathbf{D}}
$$

where $\hat{\mathbf{Q}}=\left(\hat{E}_{1}, \hat{E}_{2}, \hat{H}_{2},-\hat{H}_{1}\right)^{T}$ contains the horizontal field components. The superscript $^{T}$ indicates transposition. The operator matrix $\hat{\mathbf{A}}$ is defined as

$$
\hat{\mathbf{A}}=\left(\begin{array}{cc}
\mathbf{0}_{2} & \hat{\mathbf{A}}_{12} \\
\hat{\mathbf{A}}_{21} & \mathbf{0}_{2}
\end{array}\right)
$$

where $\mathbf{0}_{2}$ is a 2 by 2 null matrix and the matrices $\hat{\mathbf{A}}_{12}$ and $\hat{\mathbf{A}}_{21}$ are given by

$$
\begin{aligned}
& \hat{\mathbf{A}}_{12}=\left(\begin{array}{cc}
-j \omega \hat{\mu}+\partial_{1}\left(\frac{1}{\hat{\sigma}} \partial_{1} \cdot\right) & \partial_{1}\left(\frac{1}{\hat{\sigma}} \partial_{2} \cdot\right) \\
\partial_{2}\left(\frac{1}{\hat{\sigma}} \partial_{1} \cdot\right) & -j \omega \hat{\mu}+\partial_{2}\left(\frac{1}{\hat{\sigma}} \partial_{2} \cdot\right)
\end{array}\right), \\
& \hat{\mathbf{A}}_{21}=\left(\begin{array}{cc}
-\hat{\sigma}+\partial_{2}\left(\frac{1}{j \omega \hat{\mu}} \partial_{2} \cdot\right) & -\partial_{2}\left(\frac{1}{j \omega \hat{\mu}} \partial_{1} \cdot\right) \\
-\partial_{1}\left(\frac{1}{j \omega \hat{\mu}} \partial_{2} \cdot\right) & -\hat{\sigma}+\partial_{1}\left(\frac{1}{j \omega \hat{\mu}} \partial_{1} \cdot\right)
\end{array}\right) .
\end{aligned}
$$

The dot in each element of $\hat{\mathbf{A}}_{12}$ and $\hat{\mathbf{A}}_{21}$ represents a placeholder for the quantity on which the operator is applied. The source vector $\hat{\mathbf{D}}$ is defined as

$$
\hat{\mathbf{D}}=\left(\begin{array}{c}
-\hat{J}_{2}^{m}-\partial_{1} \frac{1}{\hat{\sigma}} \hat{J}_{3}^{e} \\
\hat{J}_{1}^{m}-\partial_{2} \frac{1}{\hat{\sigma}} \hat{J}_{3}^{e} \\
-\hat{J}_{1}^{e}-\partial_{2} \frac{1}{j \omega \hat{\mu}} \hat{J}_{3}^{m} \\
-\hat{J}_{2}^{e}+\partial_{1} \frac{1}{j \omega \hat{\mu}} \hat{J}_{3}^{m}
\end{array}\right)
$$

Equation (3.3) is transformed to the horizontal wavenumber domain using a 2D spatial Fourier Transformation $\tilde{u}\left(k_{1}, k_{2}, x_{3}, \omega\right)=\iint_{-\infty}^{\infty} \exp \left(j k_{1} x_{1}+\right.$ $\left.j k_{2} x_{2}\right) \hat{u}\left(x_{1}, x_{2}, x_{3}, \omega\right) d x_{1} d x_{2}$, which gives

$$
\partial_{3} \tilde{\mathbf{Q}}=\tilde{\mathbf{A}} \tilde{\mathbf{Q}}+\tilde{\mathbf{D}}
$$


where the tilde denotes the horizontal wavenumber-frequency domain. The radial wavenumber is defined as $k_{\alpha}=2 \pi \kappa_{\alpha}$ where $\kappa_{\alpha}$ is the natural wavenumber. We have assumed that the material parameters do not change laterally at the receiver level. Subsequently, an eigenvalue decomposition of the system matrix $\tilde{\mathbf{A}}=\tilde{\mathbf{L}} \tilde{\Gamma} \tilde{\mathbf{L}}^{-1}$ is used to get

$$
\tilde{\mathbf{L}}^{-1} \partial_{3} \tilde{\mathbf{Q}}=\tilde{\Gamma} \tilde{\mathbf{L}}^{-1} \tilde{\mathbf{Q}}+\tilde{\mathbf{L}}^{-1} \tilde{\mathbf{D}} .
$$

The matrix $\tilde{\boldsymbol{\Gamma}}$ is a diagonal matrix containing the eigenvalues of $\tilde{\mathbf{A}}$, whereas $\tilde{\mathbf{L}}$ consists of the eigenvectors. Equation (3.9) suggests to introduce $\tilde{\mathbf{P}}$ as

$$
\tilde{\mathbf{P}}=\tilde{\mathbf{L}}^{-1} \tilde{\mathbf{Q}} \quad \text { or } \quad \tilde{\mathbf{Q}}=\tilde{\mathbf{L}} \tilde{\mathbf{P}} .
$$

After some algebraic manipulation we obtain

$$
\partial_{3} \tilde{\mathbf{P}}=\left(\tilde{\boldsymbol{\Gamma}}-\tilde{\mathbf{L}}^{-1} \partial_{3} \tilde{\mathbf{L}}\right) \tilde{\mathbf{P}}+\tilde{\mathbf{L}}^{-1} \tilde{\mathbf{D}} .
$$

If at the decomposition level the vertical derivative of the material parameters, and hence of $\tilde{\mathbf{L}}$, vanishes and if there are no sources present at this level, equation (3.11) simplifies to a set of equations wherein the components of $\tilde{\mathbf{P}}$ are decoupled. The solution gives two downward decaying modes and two upward decaying modes. With the choices for the eigenvectors given in the appendix of this chapter, this decomposition algorithm separates Transverse Electric (TE) and Transverse Magnetic (TM) modes at the receiver level (Slob, 2009). Furthermore, the decomposed field vector $\tilde{\mathbf{P}}$ is flux-normalized. Consequently, we introduce the decomposed field vector $\tilde{\mathbf{P}}=\left(\tilde{P}^{T M+}, \tilde{P}^{T E+}, \tilde{P}^{T M-}, \tilde{P}^{T E-}\right)^{T}$, where the superscript ${ }^{+}$and the superscript ${ }^{-}$indicate downward and upward decaying components, respectively. The matrices $\tilde{\mathbf{L}}$ and $\tilde{\boldsymbol{\Gamma}}$ are given in the appendix of this chapter.

In order to obtain $\tilde{\mathbf{P}}$ in $3 \mathrm{D}$, the two horizontal electric field components and the two horizontal magnetic field components are required on a grid. In the $2 \mathrm{D}$ TM case, this requirement reduces to the horizontal inline electric field component and the horizontal crossline magnetic field component along a line. In this case, the decomposed field vector $\tilde{\mathbf{P}}$ consists of only two components, one upward decaying and one downward decaying TM component. The up-down decomposition can be done at any depth level where no sources are present. The implementation used here assumes the material parameters to be laterally constant at the depth level of decomposition but this is not a condition for the following theory (Wapenaar \& Grimbergen, 1996; Grimbergen et al., 1998). Additionally, it is necessary that the field is sampled properly. In other words, a data gap or clipped amplitudes in the vicinity of the source can not be handled by this decomposition algorithm. The presence 
of gaps can be mitigated using interpolation methods. Clipped amplitudes in the vicinity of the source, which are common in CSEM data, can be substituted by a modelled direct field. Furthermore, clipping can be avoided by using future receivers that automatically adapt their dynamic range as a function of the measured amplitude. Consequently, we ignore these limitations.

In the space-frequency domain, assuming the receivers are located at a constant depth level below the source, in 3D one downward decaying component of $\hat{\mathbf{P}}$, either TE or TM, is related with one upward decaying component of $\hat{\mathbf{P}}$, again either TE or TM, through the reflection response $\hat{R}_{0}^{+}\left(\mathbf{x}_{R}, \mathbf{x}_{R}^{\prime}\right)$ (Wapenaar et al., 2008b):

$$
\hat{P}^{-}\left(\mathbf{x}_{R}, \mathbf{x}_{S}\right)=\int_{\partial D_{R}} \hat{R}_{0}^{+}\left(\mathbf{x}_{R}, \mathbf{x}_{R}^{\prime}\right) \hat{P}^{+}\left(\mathbf{x}_{R}^{\prime}, \mathbf{x}_{S}\right) d \mathbf{x}_{R}^{\prime},
$$

where the array of receivers is represented by $\mathbf{x}_{R}$ and the source coordinates by $\mathbf{x}_{S}$. The integration is taken over all receivers. The superscript ${ }^{+}$in the reflection response indicates that its origin is a downward decaying field and the subscript 0 represents the absence of heterogeneities above the receiver level in the reflection response. In other words, $\hat{R}_{0}^{+}\left(\mathbf{x}_{R}, \mathbf{x}_{R}^{\prime}\right)$ is the scattered field response of the subsurface below the receivers due to an impulsive downward radiating source, i.e. a scattered Green's function. Equation (3.12) can be rewritten in matrix notation (Berkhout, 1982) as

$$
\hat{\mathbf{P}}^{-}=\hat{\mathbf{R}}_{0}^{+} \hat{\mathbf{P}}^{+} .
$$

Each column of the matrices contains various receiver positions for a fixed source position, while for the rows the situation is reversed. Interferometry by MDD solves equation (3.13) for $\hat{\mathbf{R}}_{0}^{+}$, for example, by least-squares inversion:

$$
\hat{\mathbf{R}}_{\mathbf{0}}^{+}=\hat{\mathbf{P}}^{-}\left(\hat{\mathbf{P}}^{+}\right)^{\dagger}\left[\hat{\mathbf{P}}^{+}\left(\hat{\mathbf{P}}^{+}\right)^{\dagger}+\varepsilon^{2} f_{\mathrm{LSQR}} \mathbf{I}\right]^{-1} .
$$

The superscript ${ }^{\dagger}$ denotes complex-conjugation and transposition and $\mathbf{I}$ is the identity matrix. The stabilization parameter $\varepsilon^{2}$ prevents instabilities in the inversion. It is scaled with a factor $f_{\mathrm{LSQR}}$, which is based on the downward decaying field. The scaling factor $f_{\mathrm{LSQR}}$ is taken as the mean of the diagonal elements of the correlation of the downward decaying field. In this way, the stabilization parameter becomes comparable for different datasets.

Assuming that the receivers are at the ocean bottom and that the decomposition into downward and upward decaying fields is done with the medium parameters of the first layer below the ocean bottom, the MDD result of equation (3.14) is the reflection response that would be measured at the 
ocean bottom if the water layer were replaced with a homogeneous half-space consisting of the same material as the first layer below the water.

When the stabilization parameter $\varepsilon^{2}$ is chosen very large, $\hat{\mathbf{P}}^{+}\left(\hat{\mathbf{P}}^{+}\right)^{\dagger}$ is much smaller than $\varepsilon^{2} f_{\mathrm{LSQR}} \mathbf{I}$ and can therefore be ignored. The term in square brackets becomes then a scaled identity matrix and the MDD-equation (equation (3.14)) can be simplified to the CC-equation

$$
\hat{\mathbf{R}}_{0}^{+}=\hat{\mathbf{P}}^{-}\left(\hat{\mathbf{P}}^{+}\right)^{\dagger},
$$

which basically retrieves $\hat{\mathbf{R}}_{\mathbf{0}}^{+}$by cross-correlation. This part can be carried out for each receiver pair separately in contrast to equation (3.14), where the whole array of receivers is necessary to solve the equation.

The choice of the stabilization parameter $\varepsilon^{2}$ can be judged by computing an error using the following error function (van der Neut et al., 2008):

$$
\operatorname{Error}(\varepsilon)=\frac{\sum_{\text {samples }}\left|\hat{A}_{\mathrm{MDD}}(\varepsilon)-\hat{A}_{\text {ref }}\right|}{\sum_{\text {samples }}\left|\hat{A}_{\mathrm{CC}}-\hat{A}_{\text {ref }}\right|},
$$

where $\hat{A}$ is the amplitude of the reflection response retrieved by multidimensional deconvolution, cross-correlation or by direct modelling, indicated with the subscripts MDD, $\mathrm{CC}$ or ref, respectively. The summation is carried out over all samples of the reflection response for the redatumed source location at the centre of the receiver array in the space-frequency domain. In other words, the error function compares the retrieved reflection response by MDD (equation (3.14)) with the directly modelled reflection response. This result is then normalized by the comparison of the retrieved reflection response by CC (equation (3.15)) with the directly modelled reflection response. The consequence of the normalization is that the Error converges to 1 for large values for the stabilization parameter because the MDD result approaches the CC solution for large values of $\varepsilon^{2}$. Solving the CC equation (equation (3.15)) retrieves the reflection response with far too low amplitudes and with phase errors. Consequently, if the values of the error function are smaller than 1 , the retrieved reflection response is better than the reflection response retrieved by CC. On the other hand, if the values of the error function are larger than 1, MDD is unstable and no useful results can be expected for this $\varepsilon^{2}$. The best result lies at the minimum of the error function.

A necessary condition to compute an error function is the availability of a directly modelled reflection response. It allows one to find the perfect stabilization parameter to investigate what is possible at best. In practice, this is not possible and therefore the error functions can only be used for 
theoretical studies. Minimizing the misfit $\sum_{I=1}^{N} \sum_{J=1}^{M}\left|\hat{\mathbf{P}}^{-}-\hat{\mathbf{R}}_{0}^{+}(\varepsilon) \hat{\mathbf{P}}^{+}\right|_{I J}$ can give an indication about a good value of the stabilization parameter in practical situations.

\subsection{Acquisition Aspects}

As stated in section 3.2, the decomposition algorithm needs properly sampled fields in order to produce correct results. Wapenaar et al. (2008b) use in their paper a sampling distance of $40 \mathrm{~m}$, which is quite dense for CSEM. Unlike for wavefields, where proper sampling is achieved with two points per wavelength, there is no sampling rule available for diffusive electromagnetic fields. It is the goal of this section to find such a rule.

$$
\text { air: } \sigma=10^{-12} \mathrm{~S} / \mathrm{m}
$$
$0 \mathrm{~m}$

\begin{tabular}{|c|c|}
\hline water: $\sigma=3 \mathrm{~S} / \mathrm{m}$ & sou \\
\hline$\nabla \nabla \nabla \nabla \nabla \nabla$ receivers & $\mathrm{fx}_{3, \mathrm{sr}} \nabla \nabla \nabla$ \\
\hline sediment: $\sigma=1 \mathrm{~S} / \mathrm{m}$ & \\
\hline
\end{tabular}

$\begin{array}{lr}\text { reservoir: } \sigma=0.02 \mathrm{~S} / \mathrm{m} & 2000 \mathrm{~m} \\ & 2050 \mathrm{~m}\end{array}$

sediment: $\sigma=0.5 \mathrm{~S} / \mathrm{m}$

Figure 3.1: Setup of the numerical modelling (not to scale): The black horizontal arrow indicates the source, white triangles the receivers. The conductivity $\sigma$ is given for each layer. The magnetic permeability $\mu$ is constant and set to its free-space value. The vertical source-receiver distance $x_{3, s r}$ is chosen $25 m$ for the conventional case and $825 \mathrm{~m}$ for the alternative case.

We expect that a denser sampling is necessary for a source vertically close to the receivers. Therefore, two different 2D Transverse Magnetic CSEMdatasets are modelled, a conventional situation with the source $25 \mathrm{~m}$ above the receivers, and an alternative case, in which the source is located $825 \mathrm{~m}$ above the receivers. In the following, the vertical distance between source and receivers is indicated as $x_{3, s r}$, where the subscript ${ }_{s}$ represents the source and the subscript $r$ the receiver. The length of the source antenna is in both situations $80 \mathrm{~m}$. The datasets are obtained in a configuration shown in Fig. 3.1 that consists from top to bottom of a half-space of air, a water layer that has a thickness of $1000 \mathrm{~m}$, a $200 \mathrm{~m}$ thick sedimentary layer, and a half-space 
of sediments. The lower half-space is intersected by a reservoir layer 1000 $\mathrm{m}$ below the sea floor. An electric source, with antenna orientation parallel to the line of receivers, generates a monochromatic signal at a frequency of $0.5 \mathrm{~Hz}$. The resulting earth response is recorded by $E_{1}$ and $H_{2}$ receivers. The subscript ${ }_{1}$ indicates inline receivers, whereas the subscript ${ }_{2}$ represents crossline receivers.

The magnitude of the two electromagnetic field components $H_{2}$ and $E_{1}$ are shown in Fig. 3.2 in a semi logarithmic plot. The conventional case $\left(x_{3, s r}=25 \mathrm{~m}\right)$ is plotted with a red line and the alternative case $\left(x_{3, s r}=825\right.$ $\mathrm{m})$ with a blue line. The amplitude is generally lower in the alternative case than in the conventional case because the source is further away from the receivers. For the same reason, the slope of the curve representing the conventional case is steeper at small offsets for both field components than in the alternative case. When the magnetic and the electric fields are compared for the conventional case, it can be seen that the electric field decays over five orders of magnitude whereas the magnetic field decays only over three orders of magnitude. This difference is less pronounced in the alternative situation because of the generally smaller dynamic range.
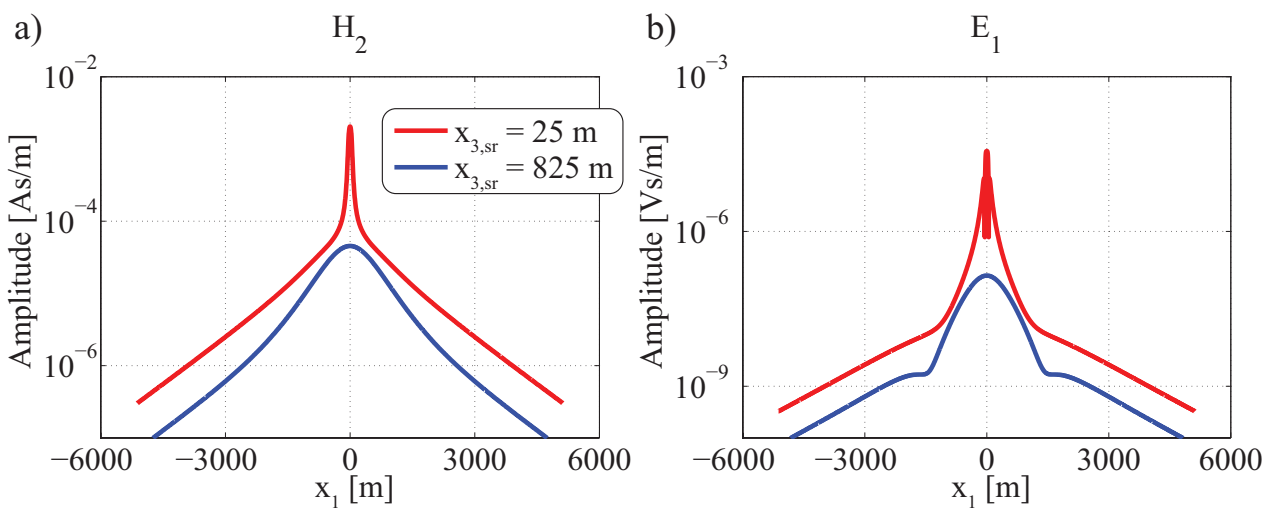

Figure 3.2: Electromagnetic fields a) $\mathrm{H}_{2}$ b) $E_{1}$ in the space domain as a function of offset for the conventional case (red) and the alternative case (blue) on a semi logarithmic plot.

The same electromagnetic fields are shown in the wavenumber domain in Fig. 3.3. Both, the electric and magnetic fields, are stronger at large wavenumbers in the conventional case than in the alternative case. In other words, the data have a larger bandwidth in the conventional situation than in the alternative situation. The reason for this can be found in the steep field gradients in the space domain for the conventional situation around zero 
offset. These steep gradients are translated to a higher field strength at large wavenumbers in the wavenumber domain.

The spatial Nyquist criterion defines the largest wavenumber $\kappa_{1, \max }$ that can be reproduced with a specific receiver sampling $d x_{1}$ :

$$
\kappa_{1, \max }=\frac{1}{2 d x_{1}} .
$$

According to equation (3.17), increasing the spacing means in the wavenumber domain a limitation of the range of the wavenumbers. Consequently, a larger spacing introduces aliasing for the large wavenumbers and can alter the data significantly. In our example, the large wavenumbers found in the conventional situation can only be recorded if $d x_{1}$ is accordingly small. When the data need to be properly sampled up to the maximum wavenumber of $0.2 \mathrm{~m}^{-1}$ as shown in Fig. 3.3, a spacing of $d x_{1}=2.5 \mathrm{~m}$ becomes necessary. The bandwidth of the conventional and the alternative datasets justify our expectation that a denser sampling will be necessary in the conventional case compared to the alternative case.
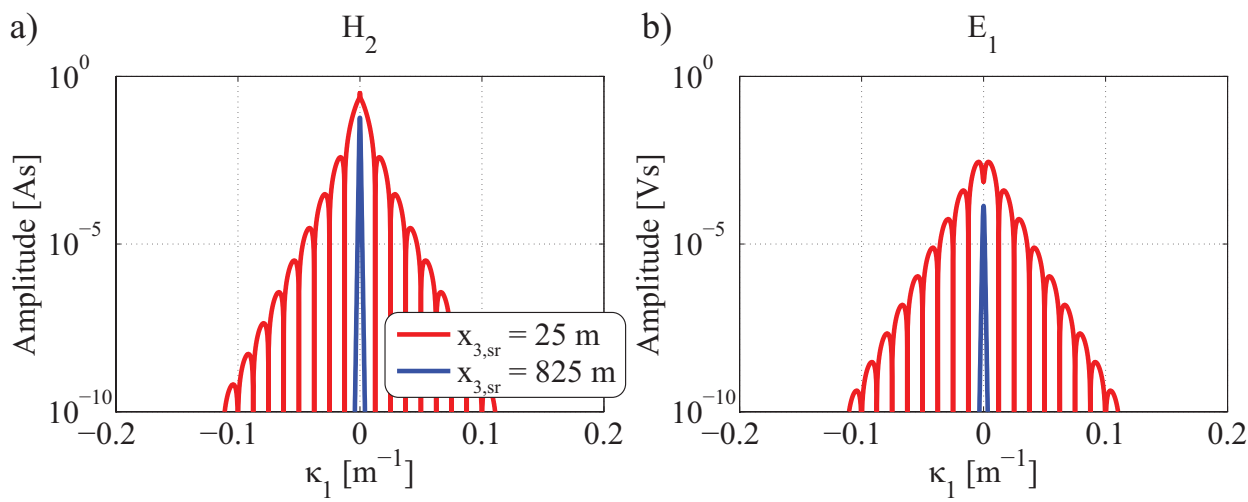

Figure 3.3: The same electromagnetic fields a) $H_{2}$ b) $E_{1}$ as in Fig. 3.2 in the wavenumber domain.

The different lobes visible in the conventional dataset are the imprint of the source with a length of $80 \mathrm{~m}$. If the sampling distance is increased to half the length of the source, in this case $40 \mathrm{~m}$, the bandwidth is limited such that only the central lobe remains. The signal of the lobes at larger wavenumbers are aliased and therefore folded to smaller wavenumbers. Due to the exponential decay of the electromagnetic fields, the lobes at larger wavenumbers are much smaller in amplitude than the central lobe and since at the edge of each lobe the field is smoothly going to zero, the resulting artefacts are negligible. In other words, the sampling distance can be increased. If the source 
length is increased, the lobes become narrower. Consequently, increasing the source length allows an increase of the sampling distance because more and more large wavenumbers can be eliminated.

If the bandwidth of the complete data is narrowed such that it is smaller than the bandwidth of the reservoir response, the response from the reservoir is altered. Therefore the question arises, how large is the bandwidth of the response from the reservoir? To answer this question a dataset without the reservoir layer was modelled for the conventional and for the alternative situation. Subtraction of the dataset with a reservoir from the corresponding dataset without a reservoir in the wavenumber domain gives the bandwidth of the reservoir response (Fig. 3.4). The bandwidth of the reservoir is much smaller than the bandwidth of the complete data in the conventional case (Note that the horizontal axis has been scaled by a factor of 100 compared to Fig. 3.3.). Also the limited bandwidth of the alternative dataset does not affect the information about the reservoir. With a bandwidth of $0.001 \mathrm{~m}^{-1}$ of the reservoir response, the spatial sampling distance can be as large as 500 $\mathrm{m}$ to still properly sample the reservoir response. Consequently, the large bandwidth in Fig. 3.3 does not originate in the reservoir response but in the direct field. Besides the direct field, also reflectors and inhomogeneities close to the receivers can cause a large bandwidth of the data.

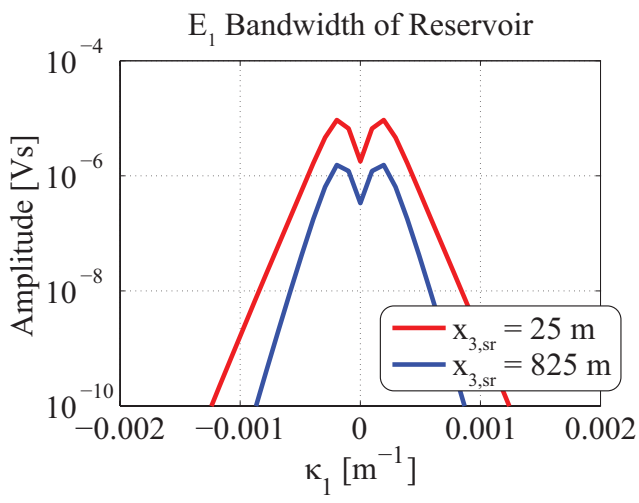

Figure 3.4: The reservoir response in the wavenumber domain. Note that the horizontal axis has been scaled by a factor of 100 and the maximum value of the vertical axis has been limited to $10^{-4}$ Vs compared to Fig. 3.3. 


\subsection{Processing}

After modelling, which was carried out in the wavenumber domain, the data were multiplied with a sinc function, which models an elongated source with constant current density. This multiplication corresponds to a convolution with a boxcar function in the space domain. Next, the datasets are inverseFourier transformed to the space domain, where samples are deleted to create more sparse datasets. Then the datasets are decomposed into upward and downward decaying fields in the wavenumber domain. Equation (3.14) is solved efficiently in the wavenumber domain. The inverse-Fourier transformed result is equivalent to $\hat{\mathbf{R}}_{0}^{+}$retrieved by MDD. The complete processing flow is shown in Fig. 3.5.

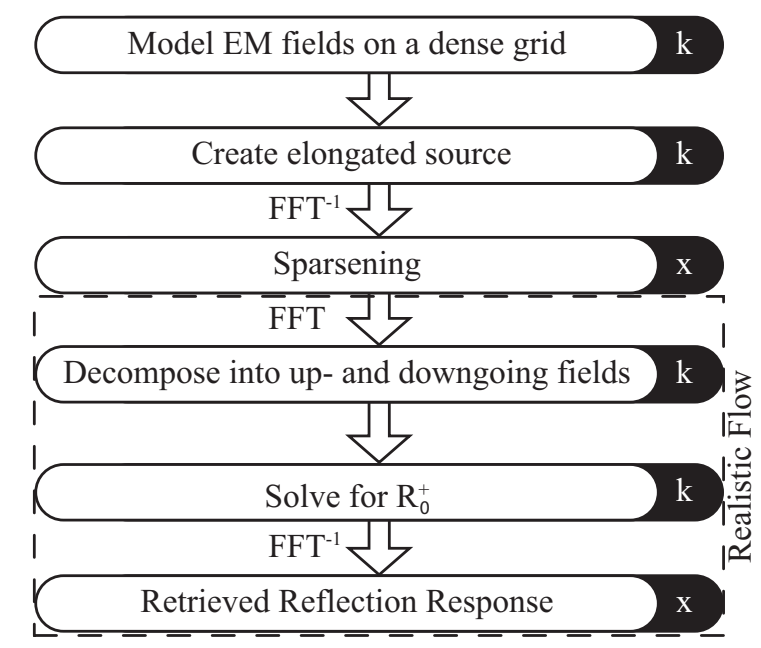

Figure 3.5: Processing flow: The dashed box labelled Realistic Flow contains all the steps that would be applicable to a real dataset. The letters $x$ and $k$ on black background indicate space and wavenumber domain, respectively.

The data for one source in the wavenumber domain correspond to the following setup of sources and receivers in the space domain: If the receiver array consists of $N$ receivers, then there are $2 N-1$ sources in the complete setup because each receiver is illuminated by $N$ sources. The remaining $N-1$ sources are out of range of this receiver. In Fig. 3.6a the layout is plotted for a setup with, for illustration purposes, $N$ equal to four receivers and seven $(2 N-1)$ sources. In the space domain, the same data for such an example correspond to a matrix like the one shown in Fig. 3.6b, where each column contains data from a common source and each row from a common receiver. Black squares indicate data and white squares mean no data. Since we are 
dealing here with a $1 \mathrm{D}$ subsurface structure and since we are not adding new offsets (Fig. 3.6), multiple sources do not actually add new data but the amount of sources influences the stability of the inversion in the space domain.
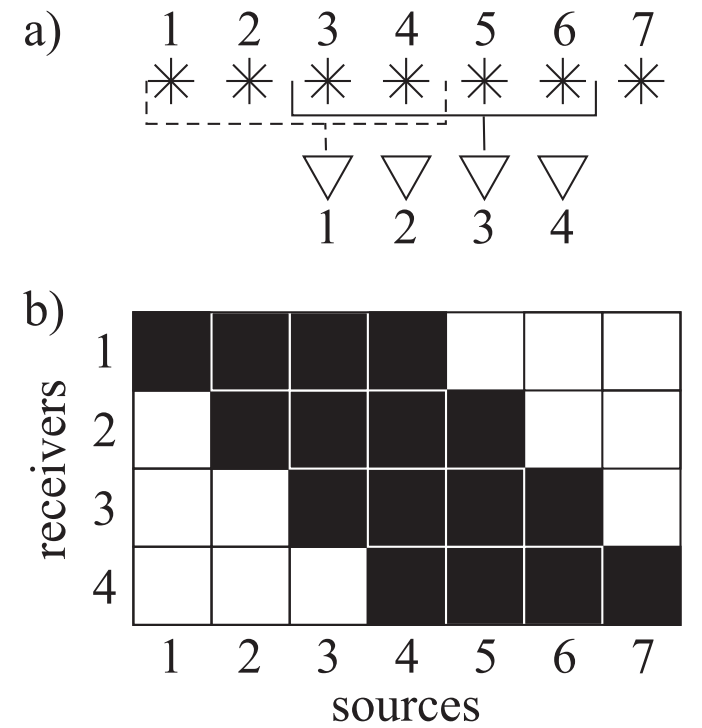

Figure 3.6: a) The data for one source in wavenumber domain corresponds to the plotted layout of sources (stars) and receivers (triangles) in the space domain. For $N$ receivers, there are $2 N-1$ sources and the distance between sources is equal to the distance between receivers. In this case $N$ is equal to 4. b) In the space domain, the data could be stored in a matrix as depicted here. Each column contains data from one specific source, each row from a specific receiver. Black squares indicate receiver positions within the range of the corresponding source, whereas white squares indicate receiver positions outside the range of the actual source. For example, receiver 1 is illuminated by sources 1 to 4 (dashed source gather in Subfigure a). Equivalently, in the first row of the datamatrix in Subfigure b), the first four datapoints are indicated in black corresponding to sources 1 to 4. Analogous, the third receiver is illuminated by sources 3 to 6 (solid source gather in Subfigure a). Consequently in the third row of the datamatrix, those datapoints are in black.

In the wavenumber domain, the inversion of the downward decaying field, which would be stored in the space domain in a matrix as shown in Fig. 3.6b, becomes a simple division and therefore equation (3.14) can be solved efficiently. A necessary condition to solve equation (3.14) in the wavenumber domain is the presence of horizontally constant medium parameters. The scaling factor $f_{\mathrm{LSQR}}$ is changed to the mean of the complete correlation matrix of the downward decaying field. Consequently, the stabilization parameter 
$\varepsilon^{2}$ in the wavenumber domain can not be compared with the stabilization parameter in space domain.

From solving equation (3.14) in the wavenumber domain it follows that when the receiver sampling distance is increased, also the source sampling distance increases. Because the up-down decomposition of the fields is independent of the source sampling and since the inversion problem remains overdetermined in any case, the coupling of the receiver-sparsening with the source-sparsening has no influence. In other words, if equation (3.14) were solved in the space domain with the described source-receiver setup and with only sparsened receivers but with dense sources, the same results as with the more efficient wavenumber domain solution would be produced.

\subsection{Results: Physical Source}

The reflection response $\hat{\mathbf{R}}_{0}^{+}$, which is retrieved after decomposition into upward and downward decaying fields and MDD, is shown in the wavenumber domain and in the space domain in Fig. 3.7 for different receiver spacings $d x_{1}$. The conventional case (dashed red line) is plotted only for negative wavenumbers or for negative offsets, whereas the alternative case (dashed blue line) is plotted only for positive wavenumbers or for positive offsets in order to achieve a clear comparison. In this way no information is lost because the retrieved reflection responses are symmetric with respect to the zero-offset position. With increasing receiver spacing $d x_{1}$ the number of receiver positions $N$ decreases because the total offset is kept constant. In the wavenumber domain as well as in the space domain, only a limited range of wavenumbers or offsets are plotted. In this section, all retrieved reflection responses are due to a redatumed source at the centre of the receiver array. Since MDD replaces the water layer by a homogeneous half-space and redatums the source position to a receiver position, the reflection responses for the conventional and the alternative case should be identical (in case of sufficiently dense sampling). The retrieved reflection responses are compared with a directly modelled reflection response (light-grey solid line), which is the exact response when the water layer is replaced by a homogeneous halfspace, the direct field is removed and the source is at a receiver position. Below each plot, the error between the retrieved and the directly modelled reflection response is shown normalized with the maximum amplitude of the directly modelled reflection response.

In Fig. 3.7a and 3.7b the receiver spacing $d x_{1}$ is equal to $2.5 \mathrm{~m}$. The two retrieved reflection responses and the directly modelled reflection response show exactly the same shape in both domains, thus verifying that MDD was 

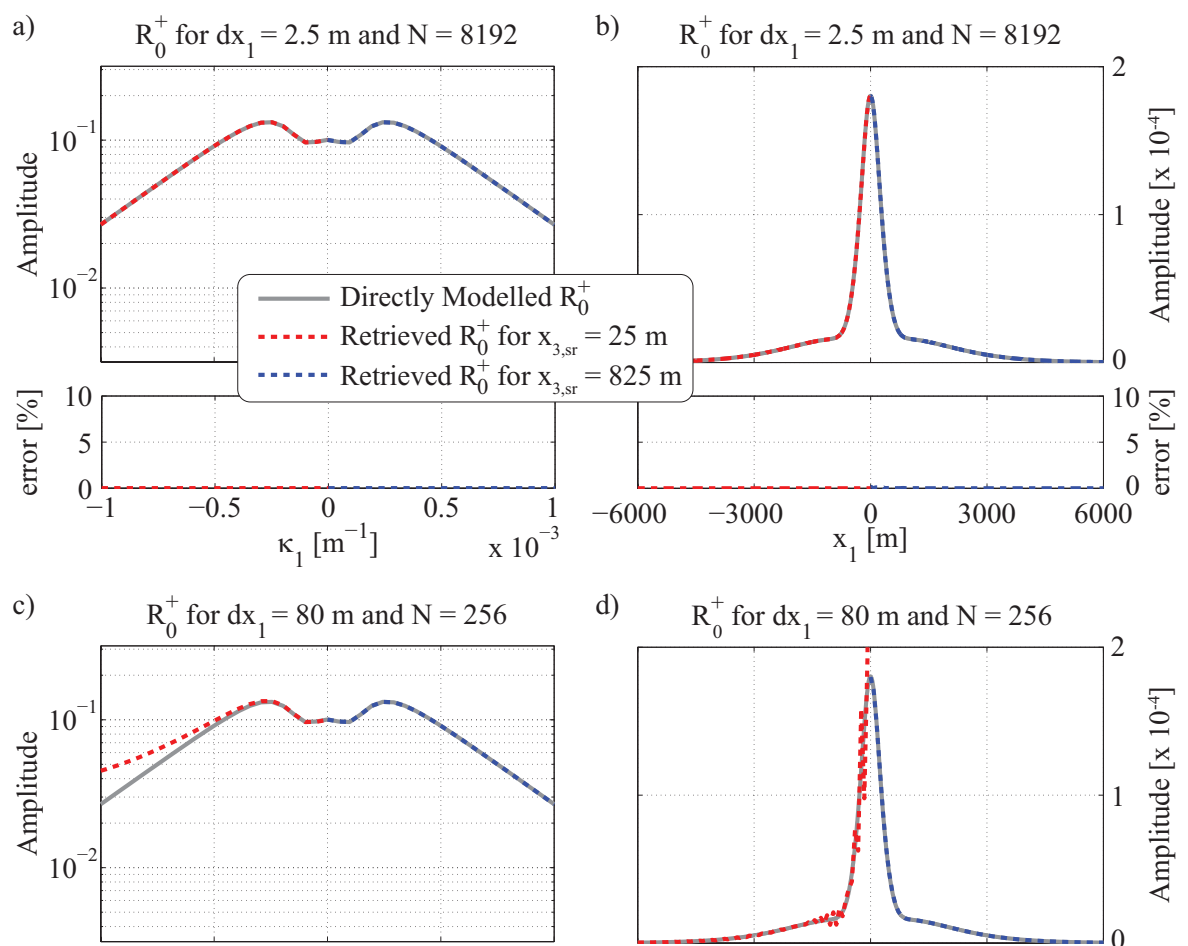

d) $\quad \mathrm{R}_{0}^{+}$for $\mathrm{dx}_{1}=80 \mathrm{~m}$ and $\mathrm{N}=256$
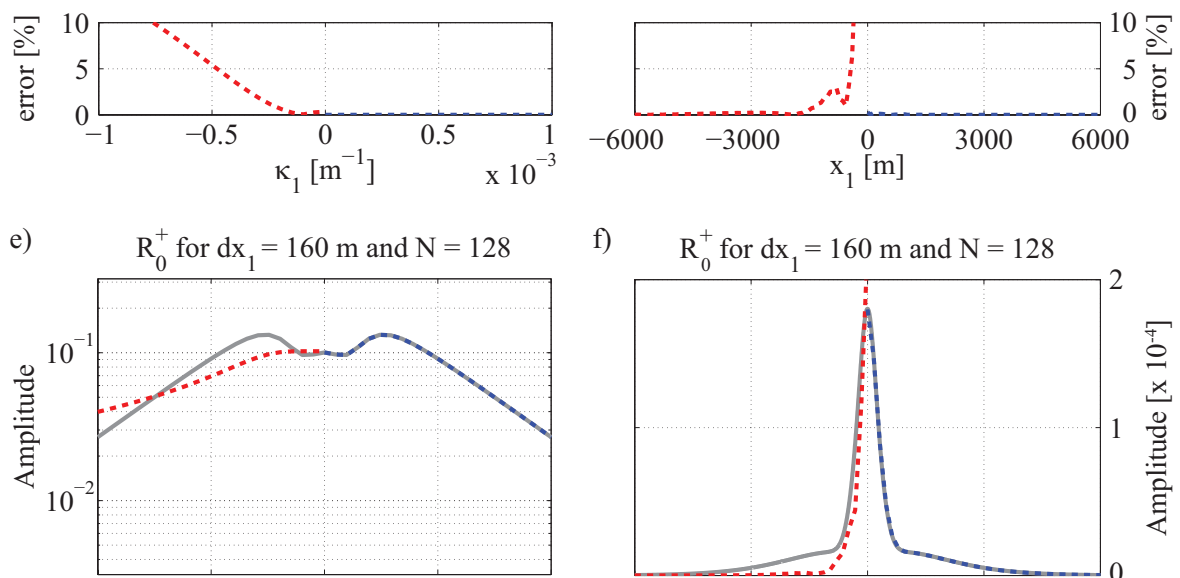

f) $\quad \mathrm{R}_{0}^{+}$for $\mathrm{dx}_{1}=160 \mathrm{~m}$ and $\mathrm{N}=128$
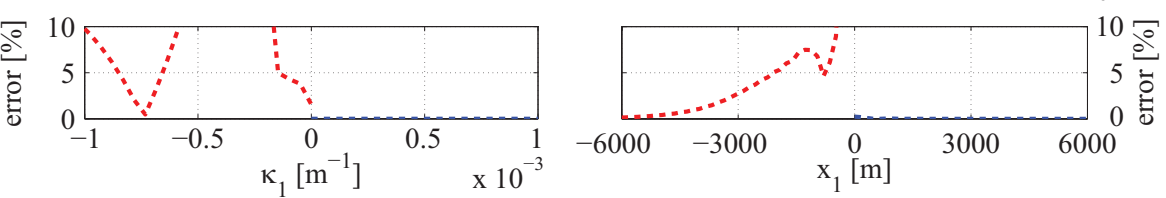

Figure 3.7: For the continuation of this Figure, see page 43. 
g)
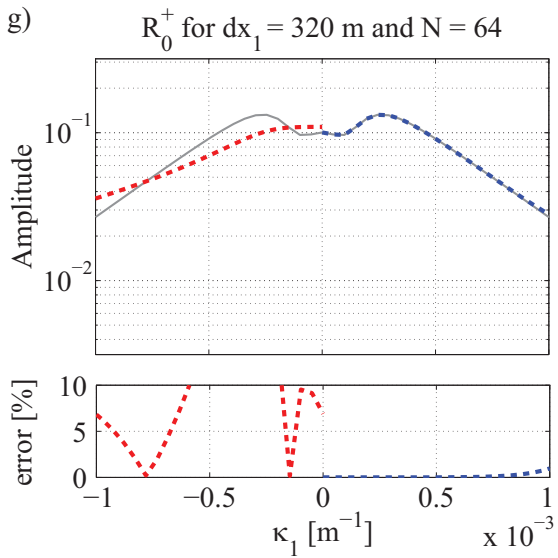

h) $\quad \mathrm{R}_{0}^{+}$for $\mathrm{dx} \mathrm{l}_{1}=320 \mathrm{~m}$ and $\mathrm{N}=64$
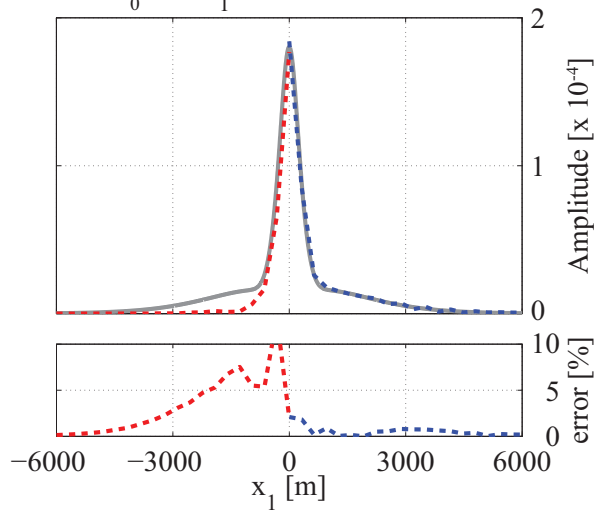

Continued Figure 3.7: Reflection responses $\hat{\mathbf{R}}_{0}^{+}$in the wavenumber domain (left column) and in the space domain (right column) as a function of offset for a)b) a receiver spacing $d x_{1}$ of $2.5 \mathrm{~m}, \mathrm{c}$ )d) $\left.80 \mathrm{~m}, \mathrm{e}\right) \mathrm{f}$ ) $160 \mathrm{~m}$ and $\mathrm{g}$ ) $\mathrm{h}$ ) $320 \mathrm{~m}$. The error between the retrieved and the directly modelled reflection response normalized with the maximum amplitude of the directly modelled reflection response is shown below each graph. Note that the plots zoom into the important part of the data and do neither show the complete wavenumber axis nor the complete axis of offsets. The spacing $d x_{1}$ and the amount of datapoints $N$ are given in the figure captions. For clarity the symmetry of the reflection responses with respect to the zero-offset position is used. Therefore, the conventional case (red) is plotted for negative wavenumbers or for negative offsets only, whereas the alternative case (blue) is plotted for positive wavenumbers or for positive offsets only. The axes span over the same range in all plots.

successful in removing the imprint of the water layer for both cases. The error is in the wavenumber domain as well as in the space domain zero, verifying the perfect retrieval. This is also true for larger spacings up to $d x_{1}=20 \mathrm{~m}$ (not shown). A spacing of $d x_{1}=40 \mathrm{~m}$ exhibits some artefacts around zero offset in the space domain in the conventional case (not shown). These artefacts are confined to an area around zero offset and do not affect larger offsets. We get a similar picture if the spacing is equal to the length of the source, in this case $d x_{1}=80 \mathrm{~m}$ (Fig. 3.7c and 3.7d). These oscillations lead to an increased error in the conventional case for small offsets. In the wavenumber domain (Fig. 3.7c), these artefacts are visible as increased amplitude for high wavenumbers. They can be significantly damped by applying a lowpass filter in the wavenumber domain. Because of this and because the overall shape and amplitude of the reflection response are retrieved properly, we neglect these artefacts and consider the reflection response as sufficiently well retrieved. 
If the spacing is further increased to $d x_{1}=160 \mathrm{~m}$, as shown in Fig. $3.7 \mathrm{e}$ and $3.7 f$, the reflection response for the conventional situation is now also for intermediate offsets not retrieved correctly. The red curve does not overlay the grey curve at all. Consequently, the error is, especially in the wavenumber domain, over a large area increased. The reason is, that the original data are aliased at this spacing. Consequently, information at high wavenumbers is folded back to small wavenumbers, leading to completely altered data at all wavenumbers. On the other hand, the alternative case $\hat{\mathbf{R}}_{0}^{+}$could be retrieved well. Increasing the spacing to $320 \mathrm{~m}$ (Fig. $3.7 \mathrm{~g}$ and $3.7 \mathrm{~h}$ ) does not change the picture. The reflection response is still well retrieved in the alternative case, whereas a retrieval is not possible in the conventional case.

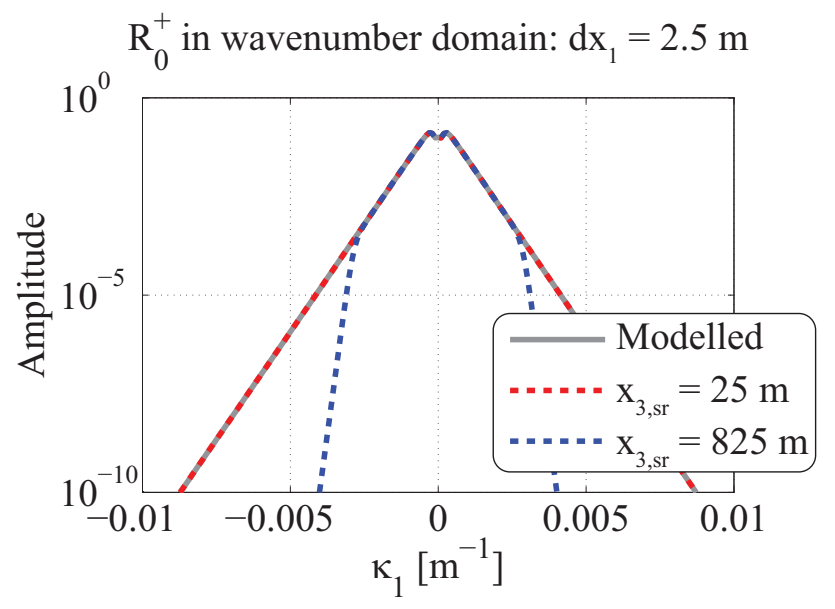

Figure 3.8: Retrieved reflection response $\hat{\mathbf{R}}_{0}^{+}$in the wavenumber domain for a larger span of wavenumbers as in Fig. 3.7 a for the densest sampling distance of $d x_{1}$ $=2.5 \mathrm{~m}$.

The retrieved reflection response for the densest sampling distance of 2.5 $\mathrm{m}$ (Fig. 3.7a and 3.7b) is shown for a larger span of wavenumbers in Fig. 3.8. Note that both axes are different than in the previous plots in the wavenumber domain. The bandwidth of the retrieved reflection response is rather small in both the conventional (dashed red line) and the alternative (dashed blue line) situation although the original fields show huge differences in bandwidth (Fig. 3.3). The bandwidth of the response from the reservoir shown in Fig. 3.4 is narrower than the bandwidth of the reflection response of Fig. 3.8 because the latter also includes the interface $200 \mathrm{~m}$ below the water bottom. In the case of the traditional situation, the retrieved reflection response overlays perfectly the directly modelled reflection response for 
the complete range of wavenumbers. The reflection response retrieved in the alternative situation coincides with the directly modelled reflection response only for small wavenumbers but deviates from the modelled reflection response at larger wavenumbers. Due to the large source-receiver distance, data at large wavenumbers are filtered out. Consequently, only information at smaller wavenumbers remains. Since the affected large wavenumbers have a relatively small amplitude, this effect is not visible in the space domain (Fig. $3.7 b)$.

\subsection{Stability analysis}

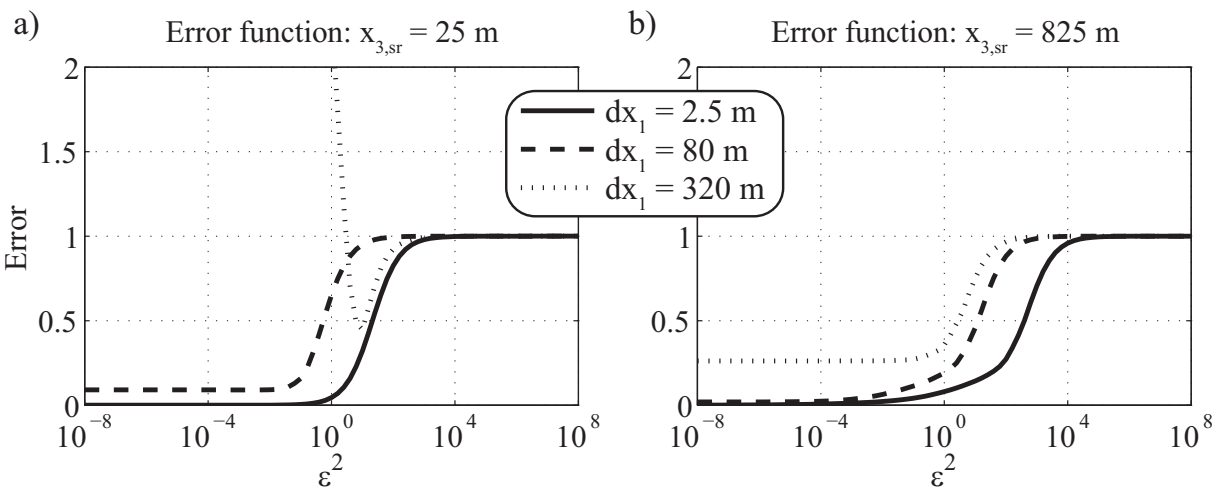

Figure 3.9: Error as a function of the stabilization parameter $\varepsilon^{2}$ for three different sampling distances $d x_{1}$. a) for the conventional case; b) for the alternative case.

A larger receiver spacing does not only affect the decomposition algorithm but also the stability of the inversion. Fig. 3.9a shows three error curves (equation (3.16)) for the conventional case for three different spacings. The densest spacing of $d x_{1}=2.5 \mathrm{~m}$ is shown by a solid line. The inversion is stable for very small values of $\varepsilon^{2}$. For larger values of $\varepsilon^{2}$ the error function converges to 1 as described in the theory section. The dashed line shows the error function for a spacing of $d x_{1}=80 \mathrm{~m}$. Also in this case the inversion is stable for very small values of $\varepsilon^{2}$. Note that in this case even the smallest error is larger than the error for a spacing of $2.5 \mathrm{~m}$. The reason is that at this spacing, the retrieved reflection response is in error around zero offset (Fig. 3.7d), which is detected by the error function. Finally, the dotted line stands for the error function for a spacing of $d x_{1}=320 \mathrm{~m}$. The minimum has now become a clearly defined trough. For too small values of the stabilization parameter the function becomes unstable (values larger than 1). The minimum error 
is larger than in the two other curves because at this spacing the retrieved reflection responses is suffering significantly from the large spacing.

The error curves for the alternative situation is shown in Fig. 3.9b. Since the bandwidth is limited in this case, a quite large spacing can be handled by the decomposition and MDD. All three curves show a broad minimum and are therefore stabilized rather easily. An error of 1 is reached for smaller values for $\varepsilon^{2}$ with increasing sampling distance. Note that in the alternative situation the smallest value of the error function at a spacing of $d x_{1}=80$ $\mathrm{m}$ is smaller than for the same spacing in the conventional case. The reason is that there are almost no artefacts around zero offset at a spacing of 80 $m$ in the alternative case, whereas the amplitude is underestimated in the conventional case. At a spacing of $d x_{1}=320 \mathrm{~m}$ some artefacts are introduced as mentioned for the conventional situation. Consequently, the minimum of the error curve rises compared to the other two curves.

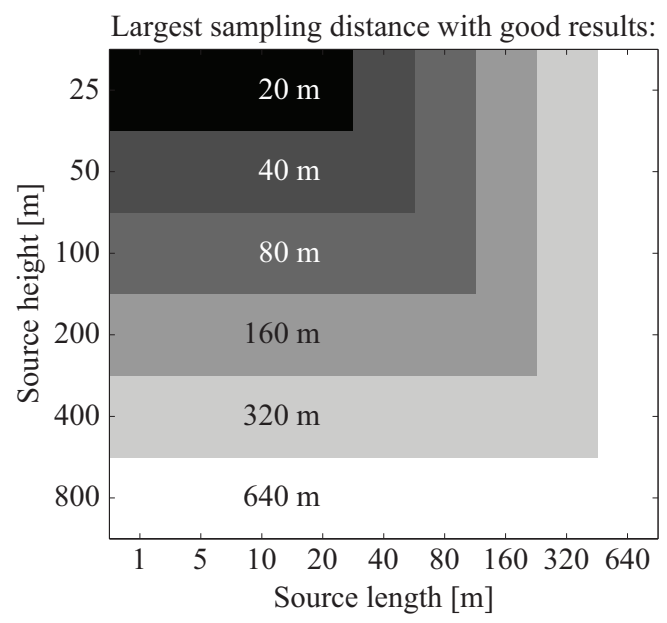

Figure 3.10: The largest sampling distance which still retrieves the reflection response well, as a function of vertical source receiver distance (source height) and length of the source antenna (source length).

Fig. 3.7 and 3.9 show that the alternative dataset can be sampled sparser than the conventional CSEM dataset because the source is vertically further away from the receivers in the alternative case. Hunziker et al. (2009) showed an empirical rule to define the proper sampling distance $d x_{1}$ as a function of the vertical source-receiver distance $x_{3, s r}$. We modify this rule with respect to two parameters: Firstly, we are able to relax this rule because in this study the stabilization parameter $\varepsilon^{2}$ is not fixed anymore for the different spacings. Secondly, we add another parameter which is the length of the source antenna 
$l$. Our findings for the largest possible receiver sampling distance for different vertical source-receiver distances (source heights) and different lengths of the source antenna (source lengths) are summarized in Fig. 3.10. It can be seen that it is possible to sample the electromagnetic fields sparser with increasing source height or increasing source length. We found that the largest possible receiver sampling distance has to be equal to or smaller than the larger of the two parameters, source length or source height

$$
d x_{1} \leq \max \left\{x_{3, s r}, l\right\} .
$$

Note that this rule assumes that instabilities around zero offset are not problematic.

\subsection{Results: Synthetic Aperture Source}

If it is not possible to pull the source antenna further away from the sea bottom (e.g. in a shallow marine situation) or if it is not desired (e.g. in order to keep a large signal strength), according to equation 3.18 the only option left is the usage of a long source antenna. To have a physically long source may not be practical. But, there is also the option of using a synthetically long source as proposed by Fan et al. (2010). They show for modelled and field data, that electromagnetic fields can be weighted and summed over many source positions to create one long source antenna. Since the source is towed by a boat over the array of receivers, there is an almost continuous record of source positions available. We create a synthetic aperture source by summing the electromagnetic field over a specific length of source positions. We give weights to the sources that are given by a Gaussian distribution function

$$
f(x)=\frac{1}{\sqrt{2 \pi \gamma^{2}}} \exp \left(-\frac{\left(x-x_{0}\right)^{2}}{2 \gamma^{2}}\right),
$$

where $x_{0}$ is the centre of the synthetic aperture source. The parameter $\gamma$ is chosen to be one eighth of the length of the synthetic aperture source. The parameter $x$ represents the source location in inline direction.

In Fig. 3.11 the reflection responses retrieved from data from a synthetic elongated source of a length of $5 \mathrm{~km}$ are shown in the wavenumber domain and in the space domain. The figure is set up in the same way as Fig. 3.7 but for different receiver spacings. In fact, the smallest receiver spacing of Fig. 3.11 is $320 \mathrm{~m}$, which is the largest receiver spacing of Fig. 3.7. For all three receiver spacing distances (i.e. $320 \mathrm{~m}, 640 \mathrm{~m}$ and $1280 \mathrm{~m}$ ) 


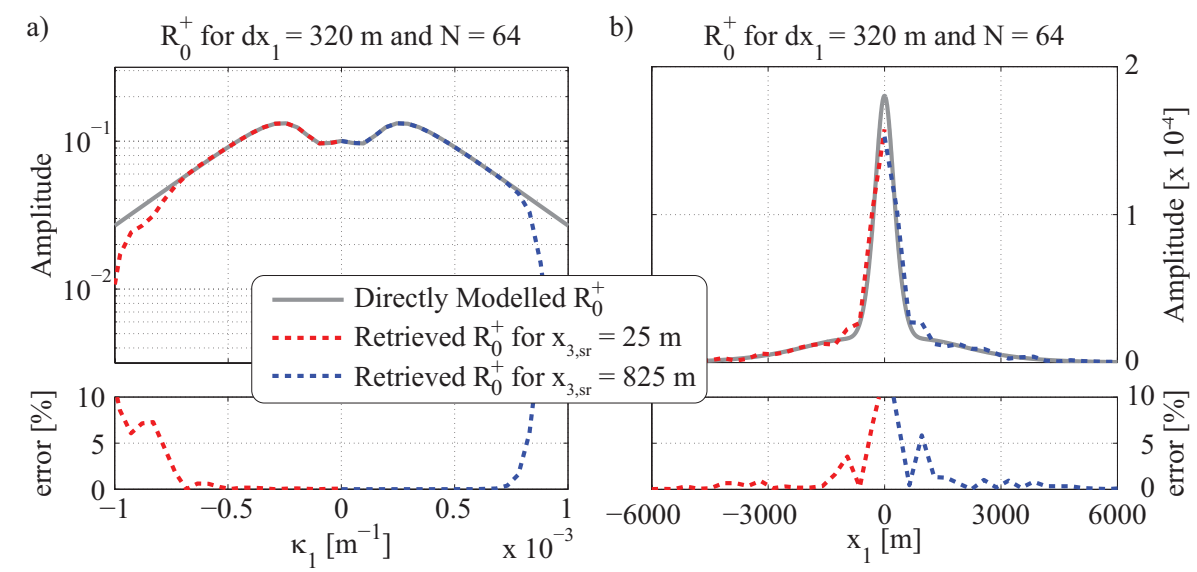

c) $\quad \mathrm{R}_{0}^{+}$for $\mathrm{dx}_{1}=640 \mathrm{~m}$ and $\mathrm{N}=32$

d) $\quad \mathrm{R}_{0}^{+}$for $\mathrm{dx}_{1}=640 \mathrm{~m}$ and $\mathrm{N}=32$
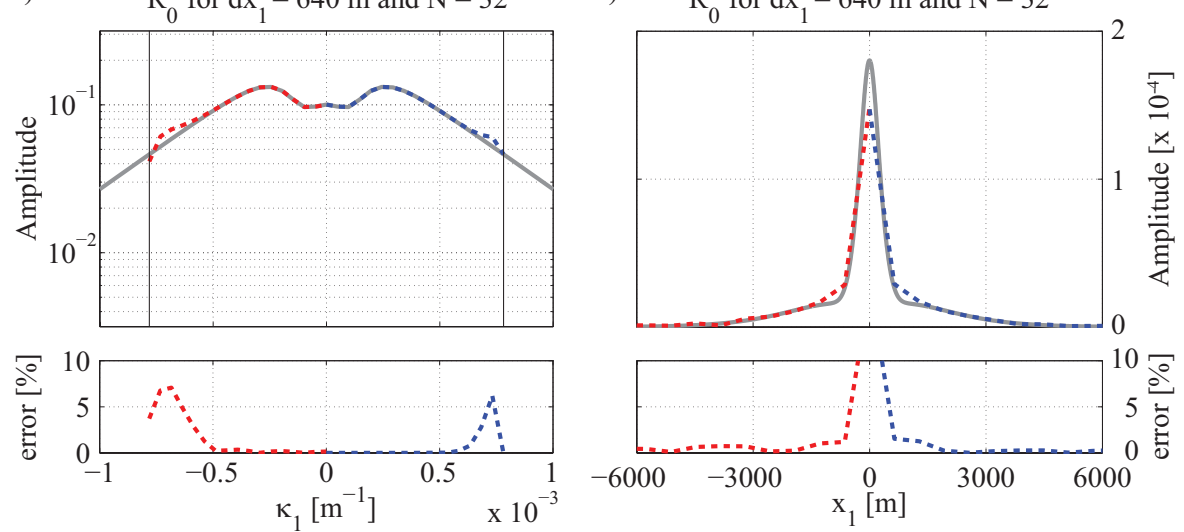

Figure 3.11: For the continuation of this Figure, see page 49.

the reflection response is retrieved perfectly within a specific bandwidth of wavenumbers. By creating a synthetically elongated source, the high wavenumbers, with respect to the complete wavenumber spectrum, are damped but not eliminated. Consequently, the high wavenumbers can be retrieved using interferometry by MDD if they remain above the noise floor in the original data. However, retrieving damped high wavenumbers leads in the deconvolution step to some instabilities seen in the space domain. Note, that the retrieved bandwidth is determined by the receiver sampling distance and not by the length of the synthetic aperture source. Thus, with increasing receiver separation, the bandwidth decreases as indicated with the vertical black lines in the wavenumber domain plots representing the spatial Nyquist frequency at $1 /\left(2 d x_{1}\right)$. The directly modelled reflection response has a much 
e)
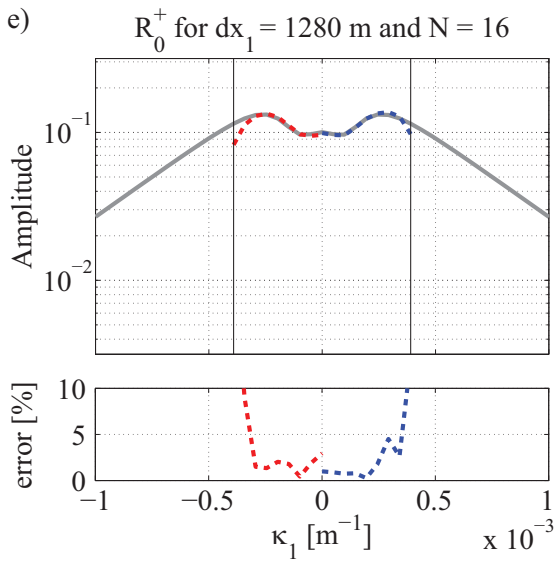

f) $\quad \mathrm{R}_{0}^{+}$for $\mathrm{dx}_{1}=1280 \mathrm{~m}$ and $\mathrm{N}=16$
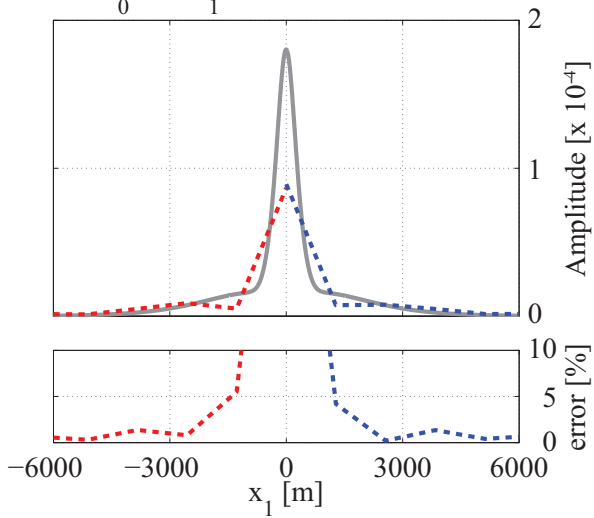

Continued Figure 3.11: Reflection responses $\hat{\mathbf{R}}_{0}^{+}$retrieved from data using the synthetic aperture source concept. The Reflection responses are shown in the wavenumber domain (left column) and in the space domain (right column) as a function of offset for a)b) a receiver spacing $d x_{1}$ of $320 \mathrm{~m}, \mathrm{c}$ )d) $640 \mathrm{~m}$ and e)f) $1280 \mathrm{~m}$. The black vertical lines in c) and e) indicate the spatial Nyquist frequency at $1 /\left(2 d x_{1}\right)$. Otherwise the same as Fig. 3.7.

wider bandwidth than the retrieved one, because it is computed using a much denser receiver spacing. This leads to a different shape in the space domain. Due to this difference in bandwidth between the directly modelled and retrieved reflection response, the error in the space domain is increasing with increasing receiver sampling distance. Still, also in the space domain, the reflection responses are retrieved well also for large receiver sampling distances within the bandwidth defined by the receiver spacing.

This shows that a physical long source can be emulated by a synthetically elongated source to overcome the strict sampling rule given in equation 3.18. In this way, large receiver sampling distances can be achieved, while keeping the current acquisition geometry. Consequently, for processing a dense receiver sampling is not necessary but one should be aware that a too large receiver sampling distance will lead to a loss of information about the reservoir response, which in turn has a negative effect on possible inversion results.

So far we have considered data for a situation with a deep ocean in order to have the option to move the source vertically in the water layer. Next we show an example of a very shallow marine situation. The model is the same as in Fig. 3.1, but the water layer is only $50 \mathrm{~m}$ thick. The source is $25 \mathrm{~m}$ above the receivers at the ocean bottom. The retrieved reflection responses 


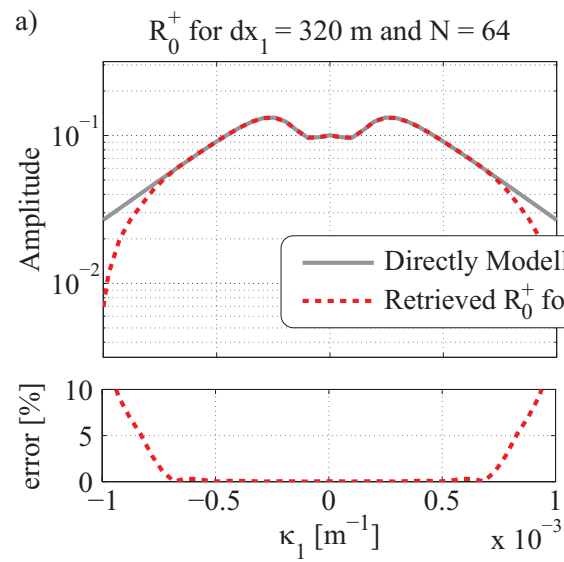

b) $\quad \mathrm{R}_{0}^{+}$for $\mathrm{dx}{ }_{1}=320 \mathrm{~m}$ and $\mathrm{N}=64$
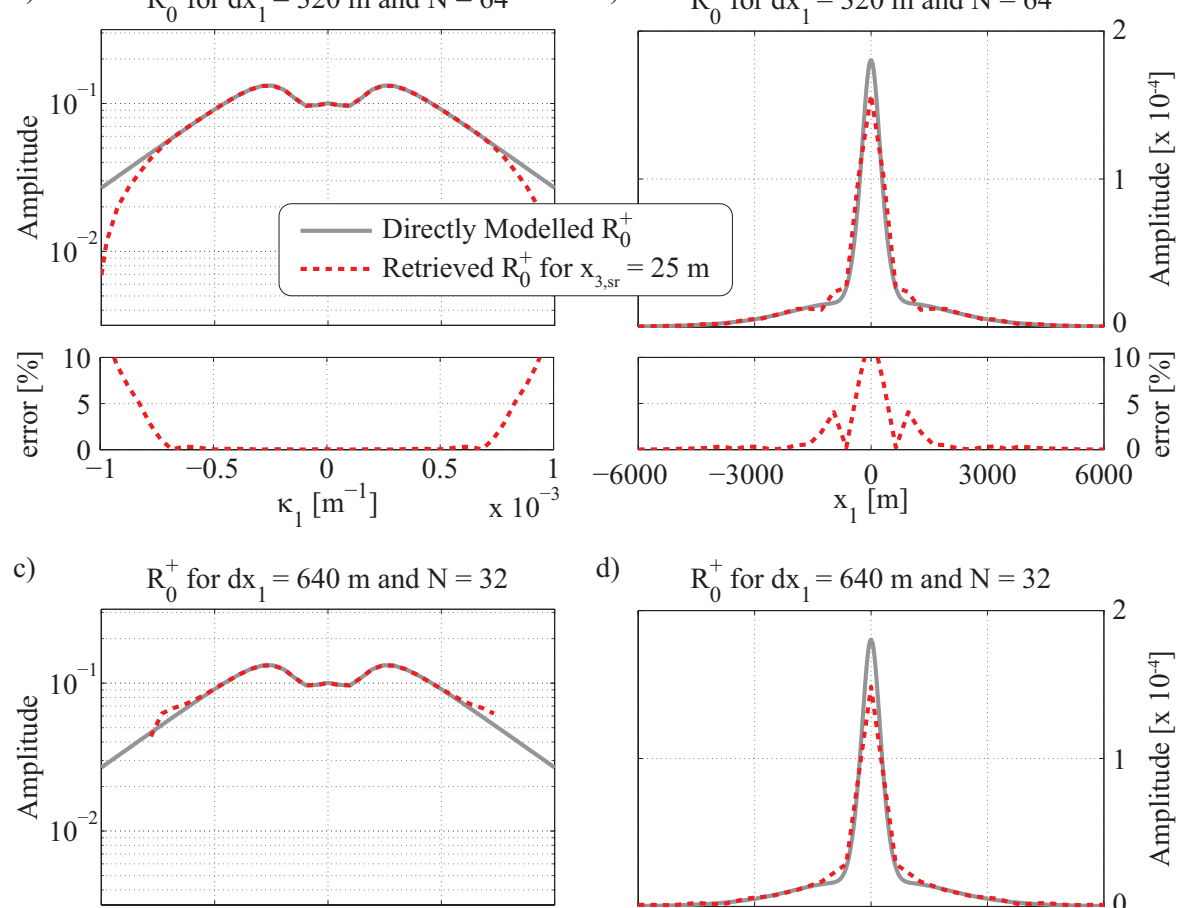

d) $\quad \mathrm{R}_{0}^{+}$for $\mathrm{dx}_{1}=640 \mathrm{~m}$ and $\mathrm{N}=32$
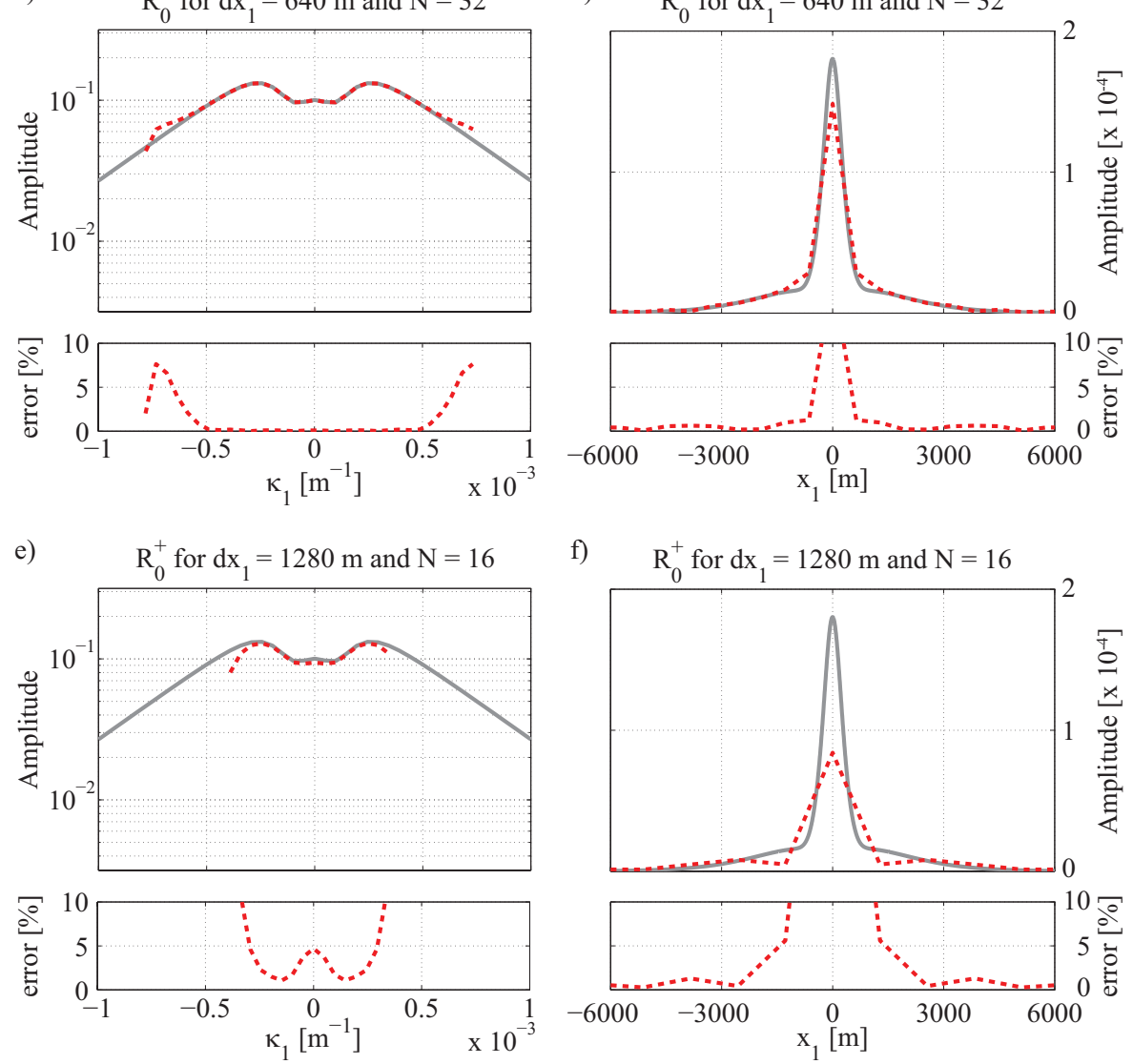

Figure 3.12: Reflection responses $\hat{\mathbf{R}}_{0}^{+}$retrieved from shallow marine data using the synthetic aperture source concept. Otherwise the same as Fig. 3.11. 
a)

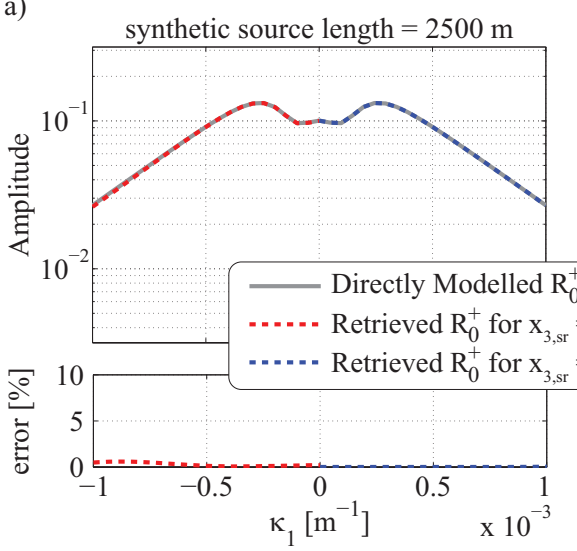

c)
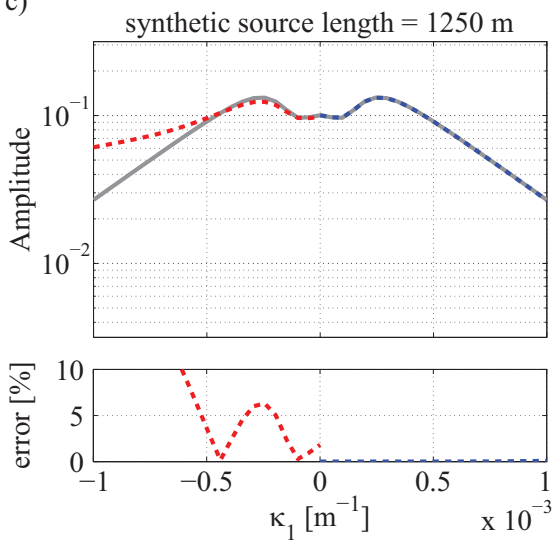

e)
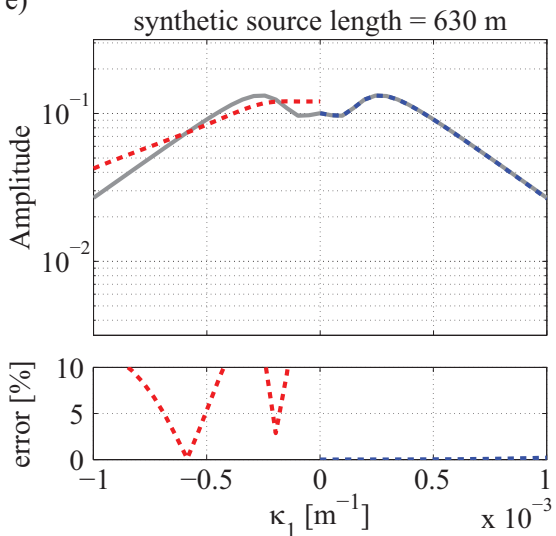

b)

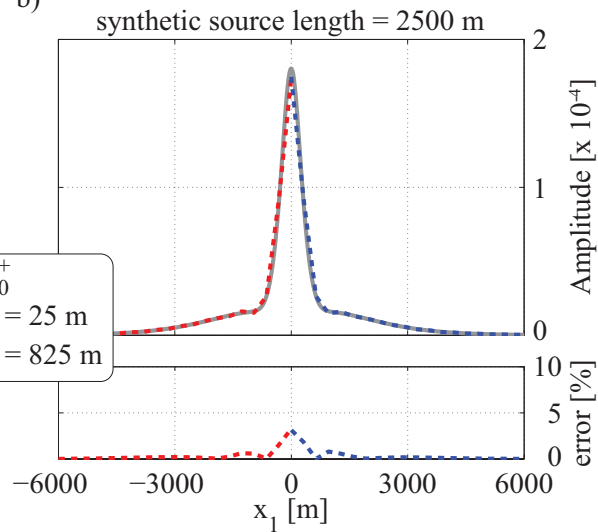

d)
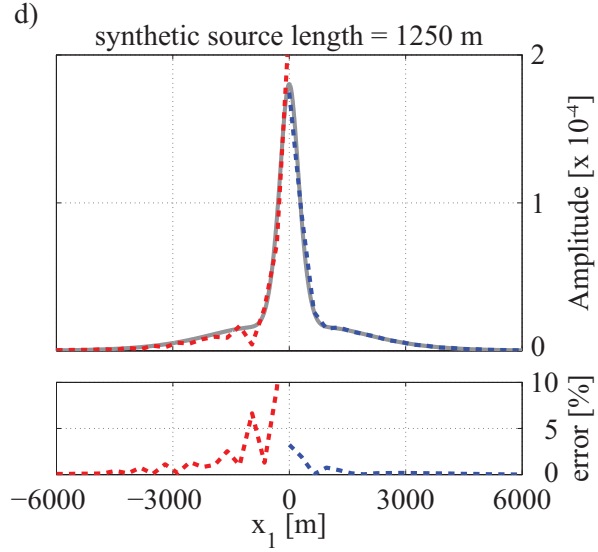

f)
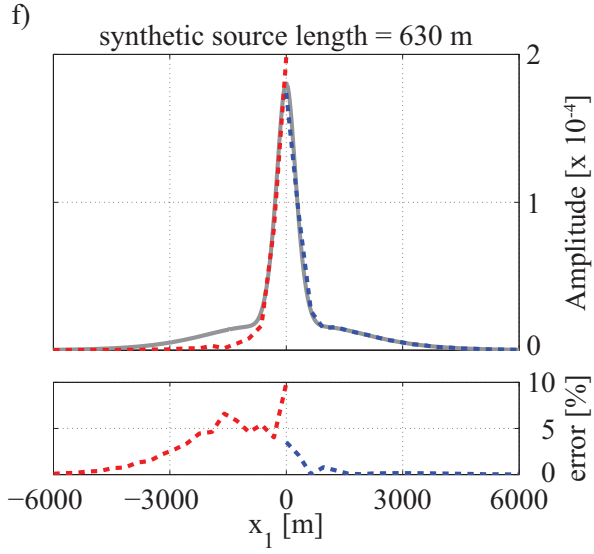

Figure 3.13: Reflection responses $\hat{\mathbf{R}}_{0}^{+}$retrieved from data sampled with a receiver spacing of $320 \mathrm{~m}$ for different synthetic aperture source lengths: a)b) $2500 \mathrm{~m}, \mathrm{c}$ )d) $1250 \mathrm{~m}$ and e)f) $630 \mathrm{~m}$. Otherwise the same as Fig. 3.11. 
for three receiver spacing distances is shown in the wavenumber domain and in the space domain in Fig. 3.12. The results are almost exactly the same as for the deep sea case (Fig. 3.11), because the retrieved reflection responses only depend on the subsurface, which is the same in both models. The waterlayer is replaced by a homogeneous halfspace and has therefore no impact on the reflection responses.

As mentioned earlier, retrieving damped high wavenumbers in the deconvolution process may lead to instabilities. By creating a smaller synthetic aperture source, less high wavenumbers are damped and, consequently, less instabilities occur. This is illustrated in Fig. 3.13a and 3.13b which are the same as Fig. 3.11a and 3.11b, but for a synthetic aperture source of only $2500 \mathrm{~m}$ length. In the wavenumber domain more high wavenumbers are retrieved properly in the conventional as well as in the alternative situation and, therefore, less artefacts appear in the space domain. Decreasing the length of the synthetic aperture source further to $1250 \mathrm{~m}$, introduces slight aliasing at high wavenumbers in the conventional case because not enough high wavenumbers are damped (Fig. 3.13c and 3.13d). A length of the synthetic aperture source of $630 \mathrm{~m}$ is in our situation definitely too short. All wavenumbers are now affected by the aliased high wavenumbers in the conventional situation. A correct retrieval of the reflection response is no longer possible in the conventional situation (Fig. 3.13e and 3.13f). The reflection response is still retrieved very well in the alternative situation, because the large vertical distance between the source and the receivers filters the high wavenumbers.

\subsection{Noise Analysis}

To test the applicability of interferometry by MDD in combination with the synthetic aperture concept under realistic conditions, we contaminated the data for the deep sea case with three different types of noise, which are random noise, receiver orientation errors and receiver positioning errors. As already mentioned, interferometry by MDD does not suffer from uncertainties in source orientation and location. Therefore we do not consider these uncertainties.

Fig. 3.14a) and b) show the reflection response retrieved from a dataset which was contaminated with random noise. The noise floor of the electric field is $10^{-11} \mathrm{Vs} / \mathrm{m}$ and the one of the magnetic field is $10^{-8} \mathrm{As} / \mathrm{m}$. Normalized with the source dipole moment we get noise levels of approximately $10^{-13}$ $\mathrm{Vs} /\left(\mathrm{Am}^{2}\right)$ and $10^{-10} \mathrm{As} /\left(\mathrm{Am}^{2}\right)$. These noise levels are two orders of magnitude larger than noise levels of current receivers (Constable, 2010). The 
various datasets with different receiver spacings were affected by the noise in a similar way. Here we show only the dataset with a receiver spacing of $320 \mathrm{~m}$ because a relatively dense sampled curve illustrates the effects of the noise better than a curve consisting only of a few datapoints. Comparing the retrieved reflection responses based on noisy data (Fig. 3.14a and b) with the reflection response retrieved from clean data (Fig. 3.11a and b) shows that even with these large noise levels, the reflection response is retrieved well. Due to the noise, the bandwidth of the alternative case is narrowed leading to a larger error at high wavenumbers. However, the average error of the retrieved reflection response increases only by $0.01 \%$ with respect to a noise free dataset. This means, that the uncertainty increases very little by adding noise.

Random receiver orientation errors of up to 10 degrees for the antenna measuring the electric field are simulated. The reflection response retrieved from that dataset (Fig. 3.14c and d) is affected significantly by this kind of noise. The average error changes from $1.24 \%$ of a noise free data to $2.84 \%$. In other words, the uncertainty of the retrieved reflection response doubles if the data are contaminated by orientation errors of up to 10 degrees. Still, within a limited bandwidth the reflection response is retrieved well.

Finally, random receiver positioning errors are modelled. In this dataset, the receivers are misplaced up to $\pm 50 \mathrm{~m}$. Similar to the receiver orientation errors, mispositioning of the receivers limits the bandwidth within which the reflection response can be retrieved properly (Fig. 3.14e and f). For this rather large mispositioning errors, the uncertainty doubles. Still, the reflection response can be retrieved well.

We have also modelled combined receiver positioning and orientation errors. The effect of both errors add up what results in a decrease of the properly retrieved bandwidth in the wavenumber domain or in a deteriorated reflection response in the space domain. Still, with random receiver positioning errors of up to $\pm 50 \mathrm{~m}$ and random receiver orientation errors of up to 10 degrees, the reflection response could be retrieved properly (not shown).

Another problem, not discussed so far, is bathymetry. The decomposition assumes that the receivers are located on a flat surface. Thus rapid, small scale variations in vertical receiver position leads to noise, which is expected to be comparable to noise from receiver positioning errors. Smooth receiver variations due to large scale changes in the topography may be handled by a decomposition algorithm for curved surfaces. Such an algorithm could be based on recently published one-way wave fields in curvilinear coordinate systems (Frijlink \& Wapenaar, 2010). The situation, where the receivers 
a)

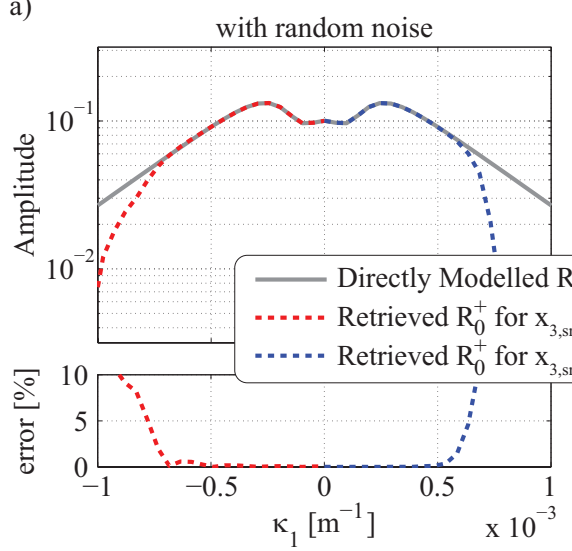

c)
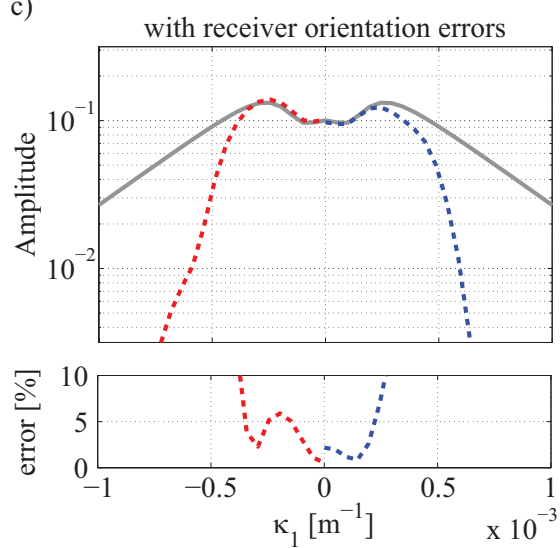

b)

b) with random noise

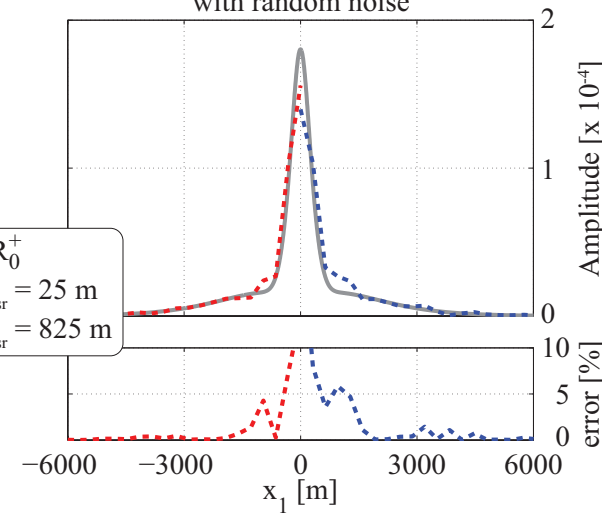

d)
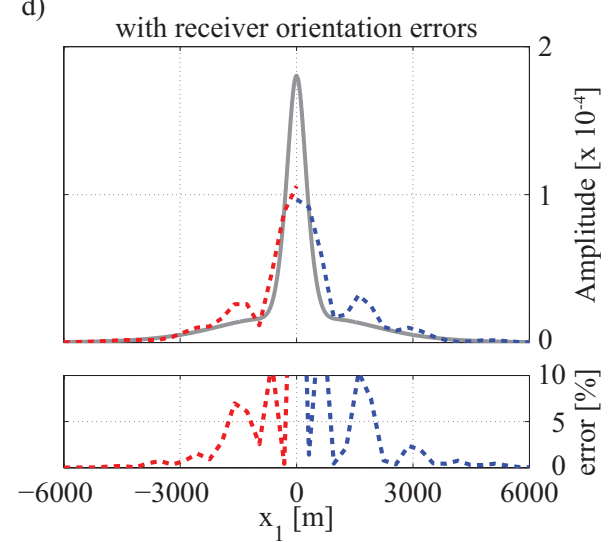

Figure 3.14: For the continuation of this Figure, see page 55.

are on a flat, but tilted surface, is ongoing research but it is assumed that decomposition works properly. In that case the decomposed fields are not purely upward or downward decaying but contain also horizontal components, such that the decomposed fields decay in a direction orthogonal to the tilted surface. When receiver height variations are large and erratic, this method will not work.

\subsection{Conclusions}

We modelled CSEM data in a marine environment for a conventional situation of a source vertically close to the receivers and an alternative situation where the source is vertically far away from the receivers for an $80 \mathrm{~m}$ long 
e)
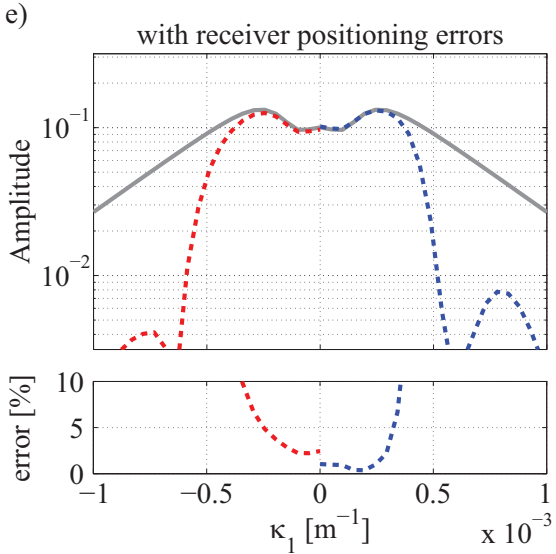

f)

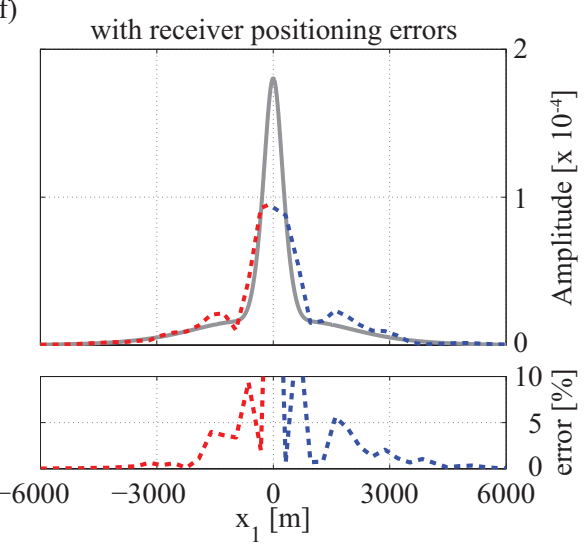

Continued Figure 3.14: Reflection responses $\hat{\mathbf{R}}_{0}^{+}$retrieved from data sampled with a receiver spacing of $320 \mathrm{~m}$ using the synthetic aperture source concept. The Reflection responses are shown in the wavenumber domain (left column) and in the space domain (right column). Below them, the error is plotted. In a) and b) are the original data contaminated with random noise with a noise floor of $10^{-11} \mathrm{Vs} / \mathrm{m}$ for the electric field and a noise floor of $10^{-8} \mathrm{As} / \mathrm{m}$ for the magnetic field. In c) and d) random orientation errors of up to 10 degrees for the receiver antenna measuring the electric field are incorporated. In e) and f) receiver positioning errors of up to $\pm 50 \mathrm{~m}$ are included.

source. We decomposed the electromagnetic fields into upward and downward decaying fields and applied interferometry by MDD, which redatums the sources to the receiver level, replaces everything above the receivers, i.e. the water layer and the air, with a homogeneous half-space and eliminates the direct field. The retrieved reflection response $\hat{\mathbf{R}}_{0}^{+}$contains only information about the subsurface below the sea bottom and is independent of the water layer and the position and orientation of the source within the water layer. Consequently $\hat{\mathbf{R}}_{0}^{+}$is the same for the conventional and the alternative situation.

A denser receiver sampling is required for the conventional case to prevent aliasing and, therefore, problems in the up-down decomposition of the fields because the bandwidth of the data is much larger when the source is vertically close to the receivers. Consequently, moving the source vertically away from the receivers allows for sparser receiver sampling. The bandwidth of the data depends also on the length of the source. Therefore, if a longer source is used, the sampling distance can be increased as well. It was found that the larger of the following two parameters, vertical distance between source and receivers and the length of the source antenna, defines the largest possible 
receiver spacing. The spacing should be equal to or smaller than the larger of the two parameters.

To realize a large source in reality may be difficult. An alternative is the application of synthetic aperture methods to conventionally acquired datasets. We tested interferometry by MDD in combination with a synthetic aperture source for receiver spacings up to $1280 \mathrm{~m}$ successfully. Theoretically, even larger receiver spacings are possible but then the bandwidth of the retrieved reflection response gets smaller than the bandwidth of the reservoir response. The synthetic aperture source limits the bandwidth of the data and allows in this way to retrieve the reflection response for severely aliased data. Is the synthetic aperture source very large, small oscillations occur in the retrieved reflection response because too many high wavenumbers are damped. On the other hand, a too short synthetic aperture source leads to improperly retrieved reflection responses due to aliasing caused by insufficient damping of high wavenumbers. Interferometry by MDD in combination with a synthetic aperture source has also been tested successfully for datasets contaminated with significant levels of random noise, receiver orientation errors and receiver positioning errors. The results of this numerical study look promising but further development may be necessary to apply this method on field data. This includes the development of a processing flow for $3 \mathrm{D}$-data as is discussed in chapter 4 , but also for example investigations on how to handle gaps in the data or bathymetry-related problems as shortly discussed above.

\subsection{Appendix A: The matrices $\tilde{\Gamma}, \tilde{\mathbf{L}}$ and $\tilde{\mathbf{L}}^{-1}$}

The matrix $\tilde{\boldsymbol{\Gamma}}$ contains the eigenvalues of $\tilde{\mathbf{A}}$ on its diagonal

$$
\tilde{\Gamma}=\left(\begin{array}{cccc}
-\tilde{\Gamma} & 0 & 0 & 0 \\
0 & -\tilde{\Gamma} & 0 & 0 \\
0 & 0 & \tilde{\Gamma} & 0 \\
0 & 0 & 0 & \tilde{\Gamma}
\end{array}\right)
$$

with $\tilde{\Gamma}=\sqrt{k_{1}^{2}+k_{2}^{2}+\hat{\sigma} j \omega \hat{\mu}}, \Re\{\tilde{\Gamma}\} \geq 0$. Note that the 4 by 4 system matrix $\tilde{\mathbf{A}}$ has only 2 different eigenvalues, the first one corresponds to the downward decaying fields, whereas the second one corresponds to the upward decaying fields.

The composition matrix $\tilde{\mathbf{L}}$ contains the eigenvectors of $\tilde{\mathbf{A}}$ in its columns. 
In this case it is set up as follows

$$
\tilde{\mathbf{L}}=\left(\begin{array}{cc}
\tilde{\mathbf{L}}_{1} & \tilde{\mathbf{L}}_{1} \\
\tilde{\mathbf{L}}_{2} & -\tilde{\mathbf{L}}_{2}
\end{array}\right)
$$

with the 2 by 2 submatrices

$$
\begin{aligned}
& \tilde{\mathbf{L}}_{1}=\frac{1}{\sqrt{2\left(k_{1}^{2}+k_{2}^{2}\right)}}\left(\begin{array}{cc}
j k_{1} \sqrt{\frac{\tilde{\Gamma}}{\hat{\sigma}}} & -j k_{2} \sqrt{\frac{j \omega \hat{\mu}}{\tilde{\Gamma}}} \\
j k_{2} \sqrt{\frac{\tilde{\Gamma}}{\hat{\sigma}}} & j k_{1} \sqrt{\frac{j \omega \hat{\mu}}{\tilde{\Gamma}}}
\end{array}\right), \\
& \tilde{\mathbf{L}}_{2}=\frac{1}{\sqrt{2\left(k_{1}^{2}+k_{2}^{2}\right)}}\left(\begin{array}{cc}
j k_{1} \sqrt{\frac{\hat{\sigma}}{\tilde{\Gamma}}} & -j k_{2} \sqrt{\frac{\tilde{\Gamma}}{j \omega \hat{\mu}}} \\
j k_{2} \sqrt{\frac{\hat{\sigma}}{\tilde{\Gamma}}} & j k_{1} \sqrt{\frac{\tilde{\Gamma}}{j \omega \hat{\mu}}}
\end{array}\right) .
\end{aligned}
$$

The inverse composition matrix, i.e. the decomposition matrix, $\tilde{\mathbf{L}}^{-1}$ is given by

$$
\tilde{\mathbf{L}}^{-1}=\frac{1}{2}\left(\begin{array}{cc}
\tilde{\mathbf{L}}_{1}^{-1} & \tilde{\mathbf{L}}_{2}^{-1} \\
\tilde{\mathbf{L}}_{1}^{-1} & -\tilde{\mathbf{L}}_{2}^{-1}
\end{array}\right)
$$

where the inverses of the submatrices are given by $\tilde{\mathbf{L}}_{1}^{-1}=-2 \tilde{\mathbf{L}}_{2}^{T}$ and $\tilde{\mathbf{L}}_{2}^{-1}=$ $-2 \tilde{\mathbf{L}}_{1}^{T}$. 


\section{Chapter 4}

\section{Electromagnetic Interferometry in three dimensions in wavenumber and space domains ${ }^{i}$}

\section{Abstract}

With interferometry applied to Controlled-Source Electromagnetic data, the direct field and the airwave and all other effects related to the air-water interface can be suppressed in a data-driven way. Interferometry allows to retrieve the scattered field Green's function of the subsurface or, in other words, the subsurface reflection response. This reflection response can then be further used to invert for the subsurface conductivity distribution. In order to perform interferometry in 3D, measurements on an areal grid are necessary. We show how this can be circumvented by using several source lines. This socalled synthesized-2D interferometry assumes that the medium is not varying in the crossline direction. In case the medium is strongly varying in all directions, full-3D interferometry needs to be applied. In this chapter, we discuss synthesized-2D and full-3D interferometry by multidimensional deconvolution in the frequency-wavenumber and in the frequency-space domains and provide examples for a layered earth model. We use the synthetic aperture source concept to damp the signal at high wavenumbers in order to allow large receiver sampling distances. We also show that interferometry indeed increases the detectability of a subsurface reservoir. Finally, we discuss the dependency of the accuracy of the retrieved reflection response on the two

${ }^{\mathrm{i}}$ This chapter has been submitted as a journal paper to Geophysics. Note that minor changes have been introduced to make the text consistent with the other chapters of this thesis. 
crucial parameters: the conductivity of the seabed at the receiver location and the stabilization parameter of the least-squares inversion.

\subsection{Introduction}

A typical marine Controlled-Source Electromagnetics (CSEM) survey consists of multicomponent ocean-bottom receiver stations and an electric source, which is towed by a boat over the receiver stations. A detailed overview of the method is given by Constable \& Srnka (2007). The low frequency signal diffuses from the source not only through the subsurface to the receivers, but also via the sea surface and directly through the water-layer. The so-called airwave is a signal that travels vertically upwards from the source, horizontally in the air along the sea surface and, eventually, vertically down to the receivers at the ocean bottom. This is the direct airwave and the part of the direct airwave that diffuses down into the subsurface and reflects off conductivity contrasts can also contribute significantly to the measured response (see several monitoring studies, e.g. Wirianto et al. (2011)). The airwave is only attenuated while travelling through the water, but not while propagating through the air. Consequently, its contribution is maximal in shallow water situations, because of the short travel path that attenuates the airwave, and at receiver stations at large horizontal distances from the source, because signals travelling along other paths have attenuated more until they are recorded at those receivers. In this way, the direct airwave is covering a possible signal from a subsurface reservoir. In contrast, the direct field contributes to the recorded field mainly at near offset receivers (Amundsen et al., 2006). Consequently, subsurface reflections can only be detected and interpreted at intermediate offsets.

In a sophisticated processing and interpretation scheme, CSEM data, i.e., the electromagnetic fields in the frequency-space domain as a function of offset from the source, are inverted for the subsurface conductivity distribution (for an overview see Avdeev, 2005). Due to the low frequency signal, CSEM alone is not able to produce a high resolution image of the subsurface. Therefore, structural information from seismics is often included in an inversion scheme (MacGregor \& Sinha, 2000; Harris et al., 2009; Key, 2009).

In contrast to seismic interferometry (Schuster, 2009; Wapenaar et al., 2010a,b), which is an established research topic, electromagnetic interferometry is a rather young area of research. The main purposes of applying interferometry by multidimensional deconvolution (MDD) to marine CSEM data are to suppress the airwave in a data-driven way and to minimize source position and orientation uncertainty. Interferometry by MDD, firstly, repla- 
ces the overburden, i.e., the medium above the receivers, with a homogeneous halfspace, secondly, suppresses the direct field and, thirdly, redatums the source to a receiver position. In other words, the scattered field Green's function of the subsurface, henceforth called subsurface reflection response, is retrieved, which is ideally free of any interactions between the air and the water-layer and, therefore, also free of the airwave. Uncertainty related to the source position and orientation is minimized, because the source is redatumed in a data-driven way to a receiver whose location is normally better known than the location of the source. We expect that an inversion scheme, which uses the reflection response as input data, features a better defined solution space, because the objective function to be minimized does not contain strong events such as the airwave or the direct field neither large uncertainties related to the source position and orientation, but ideally depends only on the subsurface response.

Theory and a 2D numerical example of electromagnetic interferometry are given in Wapenaar et al. (2008b). This technique is also known as Lorentz water-layer elimination as described by Nordskag et al. (2009). The approach consists roughly of two steps: First, the multicomponent electromagnetic fields are decomposed into downward and upward decaying components. In the second step, the reflection response is retrieved by multidimensional deconvolution. For the decomposition step, it is assumed that the data are properly sampled and that the receiver positions and orientations are known. The implementation used in this chapter requires that the receivers are located in a plane of laterally invariant material parameters. Only the material parameters at the receiver level need to be known. No information about the water-layer or the source position and orientation are required. Because the receivers are located at the ocean bottom and because the horizontal components of the electromagnetic field are continuous across the interface, one can choose to use the material parameters of the seabed or those of the water for the decomposition. Eventually, this choice determines the material parameters of the halfspace, which replaces the overburden. While van den Berg et al. (2008) chose the material parameters of the water-layer for the decomposition, we follow Wapenaar et al. (2008b) and use the material parameters of the seabed.

As already mentioned, for data-driven methods to work, the electromagnetic fields need to be properly sampled. This means that they need to be sampled sufficiently dense, for a large enough offset range and without gaps. Hunziker et al. (2012) have shown, that the largest possible receiver sampling distance depends on the height of the source above the receivers and the length of the source antenna. The receiver sampling distance is required 
to be smaller than the larger of these two parameters. Since this leads to impractically dense receiver spacings, Hunziker et al. (2012) propose to exploit the oversampling on the source side by creating synthetic aperture sources introduced by Fan et al. (2010, 2011) to overcome these sampling restrictions. They show on 2D synthetic datasets, which are contaminated with noise and with receiver position and orientation errors, the applicability of synthetic aperture sources in combination with interferometry by MDD. Synthetic aperture sources damp the high-wavenumber content, which is mainly caused by the direct field and near surface reflections, without affecting a signal from a deeper reservoir.

In this chapter, we extend the $2 \mathrm{D}$ processing scheme developed by Hunziker et al. (2012) to 3D data. To apply interferometry by MDD to 3D data, the electromagnetic field needs to be recorded on a complete areal receiver grid. Because that is not the present industry standard, we first discuss synthesized-2D interferometry, which only uses one receiver line. To compensate for the incomplete recordings, a number of source lines parallel to the line of receivers is used to create an infinitely long source in the crossline direction to allow for 2D processing. Next we discuss a full-3D processing scheme based on recordings made by a grid of receivers. Finally, we show that the reservoir detectability has increased by applying interferometry and we investigate the impact of the choice of the conductivity at the receiver level and the choice of the stabilization parameter in the least-squares inversion on the retrieved reflection response.

\subsection{Theory}

After the four horizontal electromagnetic field components are recorded, we create a synthetic aperture source in order to damp the signal at large wavenumbers and prevent aliasing of sparsely sampled data on the receiver side, as is common for CSEM data. To create such a synthetic aperture source, recordings from different source positions are weighted and subsequently summed. Note, that this does not include any steering of the source. We use the following Gaussian distribution function $f(x, y)$ as weighting function for the source positions:

$$
f(x, y)=\exp \left(-\frac{\left(x-x_{\mathrm{syn}}\right)^{2}+\left(y-y_{\mathrm{syn}}\right)^{2}}{2 l^{2} / \nu^{2}}\right),
$$

where $x$ and $y$ represent the position of the source in inline and crossline directions, respectively, and, accordingly, $x_{\text {syn }}$ and $y_{\text {syn }}$ give the centre of the 
synthetic aperture source. The parameter $l$ is the length of the source array in meters and $\nu$ is a parameter chosen empirically.

All resulting electromagnetic fields are Fourier transformed to the frequencywavenumber domain. The 2D Fourier transform of a function $\hat{u}\left(x, y, z_{1}, \omega\right)$ in the frequency-space domain is defined as $\tilde{u}\left(k_{x}, k_{y}, z_{1}, \omega\right)=\iint_{-\infty}^{\infty} \exp \left(j k_{x} x+\right.$ $\left.j k_{y} y\right) \hat{u}\left(x, y, z_{1}, \omega\right) d x d y$. The circumflex denotes the frequency-space domain and the tilde the frequency-wavenumber domain, $k_{x}$ and $k_{y}$ are the inline and crossline wavenumber, respectively, $z_{1}$ is the receiver depth (in our case at the ocean bottom) and $\omega$ is the angular frequency. The inverse Fourier transform is given by $\hat{u}\left(x, y, z_{1}, \omega\right)=1 /\left(4 \pi^{2}\right) \iint_{-\infty}^{\infty} \exp \left(-j k_{x} x-\right.$ $\left.j k_{y} y\right) \tilde{u}\left(k_{x}, k_{y}, z_{1}, \omega\right) d k_{x} d k_{y}$. A matrix multiplication of the electromagnetic field with the decomposition matrix $\tilde{\mathbf{L}}^{-1}$ given by Slob (2009) computes the decomposed field vector $\tilde{\mathbf{P}}$ at each location in the horizontal wavenumberdomain

$$
\tilde{\mathbf{P}}=\tilde{\mathbf{L}}^{-1} \tilde{\mathbf{Q}}
$$

where $\tilde{\mathbf{Q}}$ contains the field components in a vector as follows

$$
\tilde{\mathbf{Q}}=\left(\begin{array}{c}
\tilde{E}_{x}\left(k_{x}, k_{y}, z_{1}, \omega\right) \\
\tilde{E}_{y}\left(k_{x}, k_{y}, z_{1}, \omega\right) \\
\tilde{H}_{y}\left(k_{x}, k_{y}, z_{1}, \omega\right) \\
-\tilde{H}_{x}\left(k_{x}, k_{y}, z_{1}, \omega\right)
\end{array}\right) .
$$

The vector containing the decomposed fields is organized as follows

$$
\tilde{\mathbf{P}}=\left(\begin{array}{c}
\tilde{P}^{T M+}\left(k_{x}, k_{y}, z_{1}, \omega\right) \\
\tilde{P}^{T E+}\left(k_{x}, k_{y}, z_{1}, \omega\right) \\
\tilde{P}^{T M-}\left(k_{x}, k_{y}, z_{1}, \omega\right) \\
\tilde{P}^{T E-}\left(k_{x}, k_{y}, z_{1}, \omega\right)
\end{array}\right) .
$$

The superscripts + and - indicate downward and upward decaying modes, respectively, and the superscripts $T M$ and $T E$ represent transverse magnetic and transverse electric modes, respectively. By performing the decomposition in the frequency-wavenumber domain, we assume that the medium parameters are laterally invariant at the receiver level, thus at the level where the Fourier transform was computed. There are no restrictions concerning the medium above and below the receiver level, but the separation of TM- and TE-mode is restricted to the receiver level for any general medium.

We split the vector $\tilde{\mathbf{P}}$ given in equation 4.4 in a downward decaying part $\tilde{\mathbf{P}}^{+}$consisting of the first two elements and in an upward decaying part $\tilde{\mathbf{P}}^{-}$consisting of the last two elements. After a Fourier transform to the 
frequency-space domain, the two parts can be related to each other by the reflection response of the subsurface $\hat{\mathbf{R}}_{\mathbf{0}}$ as follows

$$
\hat{\mathbf{P}}^{-}=\hat{\mathbf{R}}_{\mathbf{0}} \hat{\mathbf{P}}^{+}
$$

where $\hat{\mathbf{P}}^{+}$and $\hat{\mathbf{P}}^{-}$are matrices with each column containing the receiver array for one source position and each row containing all the source positions for one receiver position (Berkhout, 1982). The reflection response $\hat{\mathbf{R}}_{\mathbf{0}}$ is a matrix set up in a similar way: Each column contains the receiver array for one redatumed source located at the receiver level and vice versa for the rows. The subscript 0 in the reflection response indicates that $\hat{\mathbf{R}}_{\mathbf{0}}$ gives the reflection response of the medium below the receivers while the medium above the receivers is homogenized. In other words, $\hat{\mathbf{R}}_{\mathbf{0}}$ does not contain any reflections from above the receiver level. The reflection response of the subsurface can be retrieved from equation 4.5 by inversion of the downward decaying field. This process is called interferometry by multidimensional deconvolution (MDD).

Assuming a laterally invariant medium, equation 4.5 can be written in the frequency-wavenumber domain as a set of scalar equations:

$$
\begin{aligned}
& \tilde{P}^{T M,-}\left(k_{x}, k_{y}, z_{1}, \omega\right)=\tilde{R}_{0}^{T M, T M}\left(k_{x}, k_{y}, z_{1}, \omega\right) \tilde{P}^{T M,+}\left(k_{x}, k_{y}, z_{1}, \omega\right) \\
& +\tilde{R}_{0}^{T M, T E}\left(k_{x}, k_{y}, z_{1}, \omega\right) \tilde{P}^{T E,+}\left(k_{x}, k_{y}, z_{1}, \omega\right), \\
& \tilde{P}^{T E,-}\left(k_{x}, k_{y}, z_{1}, \omega\right)=\tilde{R}_{0}^{T E, T M}\left(k_{x}, k_{y}, z_{1}, \omega\right) \tilde{P}^{T M,+}\left(k_{x}, k_{y}, z_{1}, \omega\right) \\
& +\tilde{R}_{0}^{T E, T E}\left(k_{x}, k_{y}, z_{1}, \omega\right) \tilde{P}^{T E,+}\left(k_{x}, k_{y}, z_{1}, \omega\right) .
\end{aligned}
$$

The two superscripts of the reflection response indicate the mode of the receiver and the source, respectively. Since we assumed a laterally invariant medium, the TM-mode and the TE-mode are decoupled (Kong, 1986; Jones, 1994). Thus, the mode-conversions, i.e. the upward decaying TM-mode generated by the downward decaying TE-mode and vice versa, do not exist. Therefore, the cross-terms $\tilde{R}_{0}^{T M, T E}$ and $\tilde{R}_{0}^{T E, T M}$ vanish, simplifying equations 4.6 and 4.7 as follows:

$$
\begin{aligned}
\tilde{P}^{T M,-}\left(k_{x}, k_{y}, z_{1}, \omega\right) & =\tilde{R}_{0}^{T M}\left(k_{x}, k_{y}, z_{1}, \omega\right) \tilde{P}^{T M,+}\left(k_{x}, k_{y}, z_{1}, \omega\right), \\
\tilde{P}^{T E,-}\left(k_{x}, k_{y}, z_{1}, \omega\right) & =\tilde{R}_{0}^{T E}\left(k_{x}, k_{y}, z_{1}, \omega\right) \tilde{P}^{T E,+}\left(k_{x}, k_{y}, z_{1}, \omega\right) .
\end{aligned}
$$

The double superscripts of the reflection response have been merged into one superscript, because they are equal anyway in the absence of modeconversions. In equations 4.8 and 4.9 , the matrix multiplication of equation 4.5 has become a simple elementwise multiplication. Hence, by division of 
the upward decaying field by the downward decaying field in the frequencywavenumber domain, the reflection response of the subsurface can be retrieved, thus avoiding a matrix inversion. As mentioned before, solving for the reflection response in the frequency-wavenumber domain assumes a laterally invariant medium, i.e., a layered earth. In case the medium is varying laterally, the reflection response needs to be retrieved in the frequency-space domain by inverting equation 4.5. Note that the components of the vectors in equation 4.5 vary between the different cases of interferometry by MDD presented in the next sections. The precise equations are given in the according sections of this chapter.

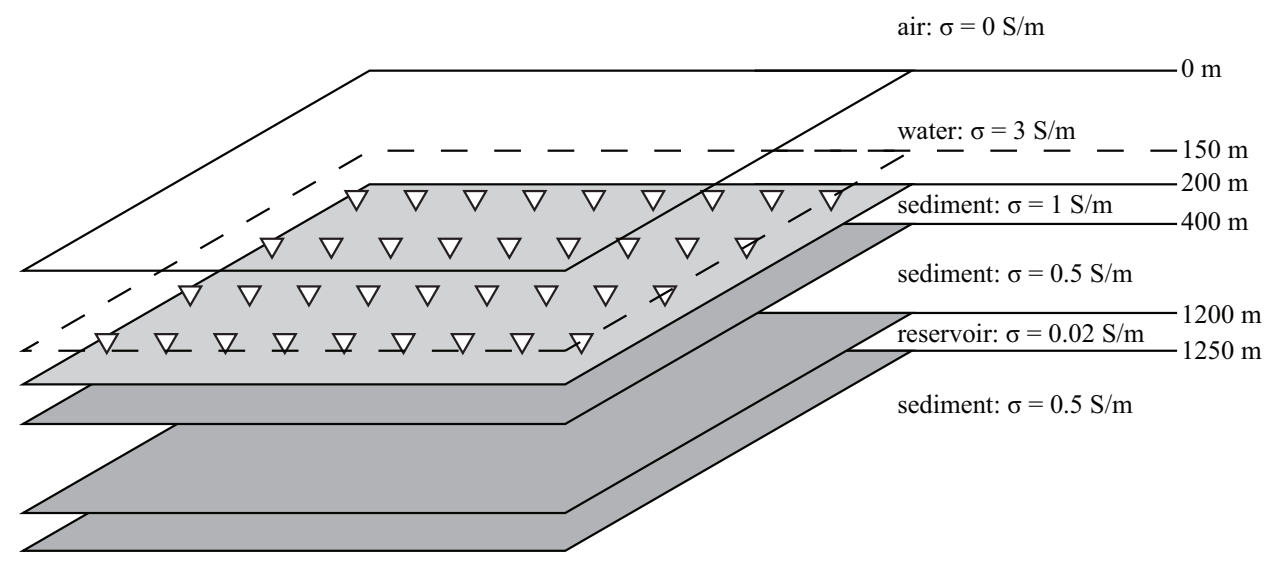

Figure 4.1: Model used for synthetic data. Transparent interface is the sea surface, light grey interface the sea bottom. All other interfaces in the subsurface are coloured dark grey. The source is located $50 \mathrm{~m}$ above the sea bottom in the plane indicated by the dashed line. Note that the conductivity does not change across the dashed plane. The receivers are located at the sea bottom (white triangles).

\subsection{Model}

Throughout the chapter we use the same subsurface model to create the synthetic data. The model consists of a halfspace of air, a shallow water-layer of $200 \mathrm{~m}$ thickness and a layered halfspace of sediments, which is intersected by a $50 \mathrm{~m}$ thick reservoir layer at $1 \mathrm{~km}$ depth below the ocean bottom. The according conductivity values and the depth of the interfaces are given in Figure 4.1. The receivers are located at the ocean bottom (white triangles) recording the four horizontal components of the electromagnetic field $E_{x}, E_{y}$, $H_{x}$ and $H_{y}$. The source, an inline-oriented antenna emitting an electric field, 
is $50 \mathrm{~m}$ above the ocean bottom (located in the dashed plane). The frequency of the source signal is $0.5 \mathrm{~Hz}$. The data are modelled in the frequencywavenumber domain using an analytical code. After a numerical Fourier transform, the data are regridded on a sparser grid with a receiver separation of $640 \mathrm{~m}$ in the inline and crossline directions and with a maximum offset of $20 \mathrm{~km}$ before further processing.

\subsection{Synthesized-2D interferometry in the frequen- cy-wavenumber domain}
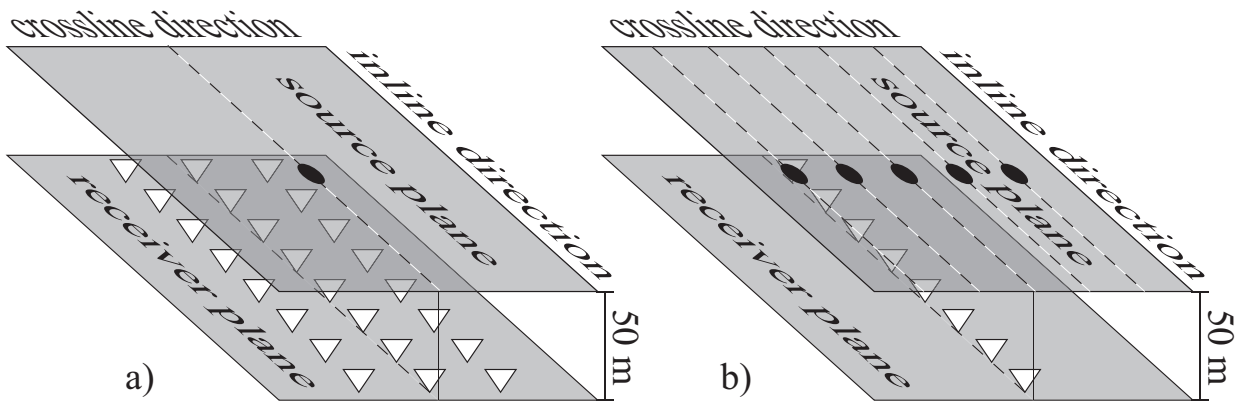

Figure 4.2: a) Full-3D acquisition geometry: The receivers (white triangles) are located on a grid in the receiver plane. The source (big black ellipse) is located above the receivers in the source plane. b) Synthesized-2D acquisition geometry: There is only one line of receivers in the receiver plane, but several sources are located in the source plane, which are excited after each other.

In order to perform a decomposition of the data into upward and downward decaying fields as part of the interferometry procedure, recordings on a grid of receivers are necessary (Figure 4.2a), which is not common in CSEM surveys. To the authors' knowledge, standard CSEM acquisition consists of one or a few lines of receivers. The 2D decomposition algorithm used by Hunziker et al. (2012) works with line-data, but also assumes the field to diffuse in $2 \mathrm{D}$. This means, the source is assumed to be infinitely long in the crossline direction. Because the receivers are in the near field of the source, this 2D decomposition algorithm applied to 3D data on a line does not produce anything meaningful. In order to apply this 2D decomposition algorithm, the data need to be converted from 3D to 2D by creating an infinitely long source in the crossline direction. This can be done by integrating in the crossline direction over a comprehensive set of source locations acquired after each other assuming the medium to be laterally invariant in the crossline 
direction. This corresponds to an acquisition geometry of several source lines off the receiver line as shown in Figure 4.2b. Naturally, using more sources improves the quality of the synthetically infinitely long source in the crossline direction. Through this integration, the TE-mode part of the electromagnetic field, and therefore also the airwave, are eliminated. If the integration is successful, the components $E_{y}$ and $H_{x}$ vanish, because those fields are odd functions of y-offset. We call this acquisition geometry and processing synthesized-2D, because the 2D-data derived from the 3D-dataset is not perfectly two-dimensional. In order to create perfect $2 \mathrm{D}$ data, an infinitesimally dense source line spacing for an infinite offset range is required.
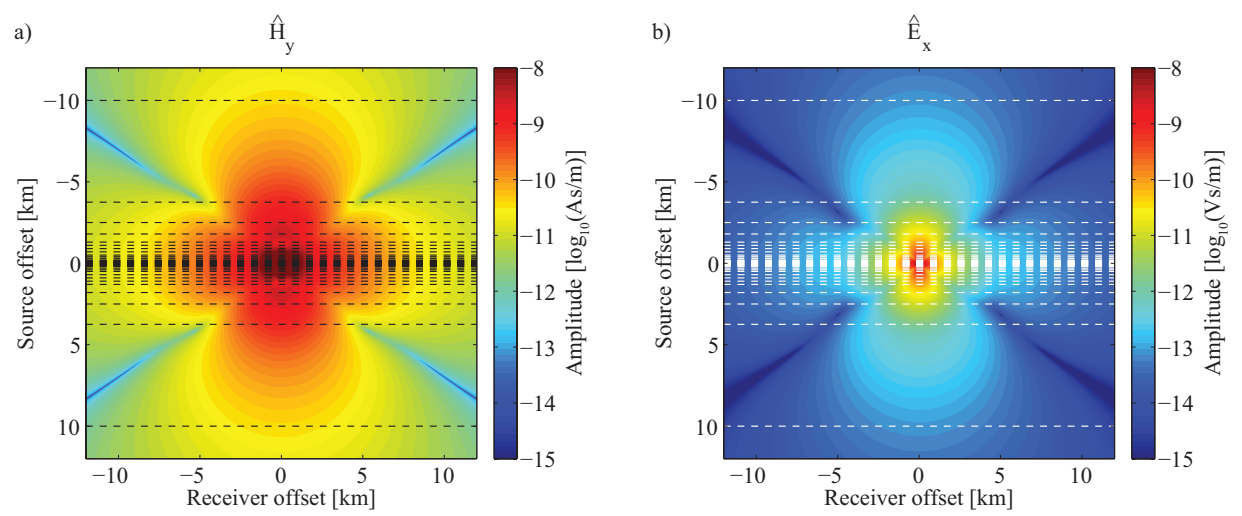

Figure 4.3: The electromagnetic field: a) $H_{y}$ and b) $E_{x}$ for the model shown in Figure 4.1 using the synthesized-2D acquisition geometry (Figure 4.2b). The horizontal axis shows the receiver offset from the source position in inline direction. The vertical axis shows the source offset from the receiver line. The source spacing as well as the receiver spacing are $10 \mathrm{~m}$. The colour indicates the amplitude of the electromagnetic fields on a logarithmic scale. 35 source positions, which are sufficient to produce an infinitely long source in crossline direction, are indicated with black dashed lines for $H_{y}$ and with white dashed lines for $E_{x}$. Two out of these 35 source positions are lying outside the offset range shown at -20 and $20 \mathrm{~km}$.

The $2 \mathrm{D}$ decomposition algorithm requires only the two non-zero components of the electromagnetic field instead of the four components for the 3D algorithm. These are the $H_{y}$ and the $E_{x}$ components. Recordings of one receiver line for a set of source positions in the crossline direction are shown for these two components in Figure 4.3. The horizontal axis corresponds to a dense line of receivers (receiver spacing of $10 \mathrm{~m}$ ) whose recordings are shown for a series of source offsets in the crossline direction separated by the same distance. Note that this very dense receiver and source spacing is only for illustration purposes. The receiver spacing used in all examples 
is $640 \mathrm{~m}$. Numerical experiments have shown that in our configuration 35 source lines are sufficient to properly create an infinitely long source in the crossline direction by integration. From those source lines, 33 out of 35 are indicated by black dashed lines for $H_{y}$ and white dashed lines for $E_{x}$. Two more source lines at -20 and $20 \mathrm{~km}$ crossline offsets are located outside the plotted offset range. Assuming a standard noise floor of about $10^{-15} \mathrm{Vs} / \mathrm{m}$ in the electric field (Constable, 2010), the amplitude of the signal is in our example approximately one order of magnitude above the noise floor at that distance from the source. Figure 4.3 indicates that close to the receiver array a denser source spacing in crossline direction is necessary than farther off. In fact, we start with a source spacing of $40 \mathrm{~m}$ which gradually increases to $10 \mathrm{~km}$ for locations farther off the receiver line. This variable source spacing captures the steep decay of the electromagnetic field close to the receiver line caused by the direct field and at the same time reduces the amount of source positions by the large source sampling distance far away from the receiver line.

The amplitude and phase of the resulting 2D electromagnetic fields are shown in Figures $4.4 \mathrm{a}$ and $4.4 \mathrm{~b}$ with a dashed red curve. The solid blue curve in the same figures shows a 2D TM-mode field that was analytically modelled in the wavenumber-domain and subsequently Fourier transformed to the space-domain. The blue and the red curve are quite similar in amplitude and phase for small and intermediate offsets, i.e., up to $5 \mathrm{~km}$, but deviate from each other at large offsets. Consequently, the 2D-field can only be reconstructed for a limited offset range. Theory predicts, that if the fields are infinitesimally densely sampled for an infinite offset range, the 2D-field will be reconstructed properly. In our case, sparsely sampled datapoints over a limited offset range are used. Experiments have shown that denser sampling does not increase the well-reconstructed offset range significantly. Consequently, only datapoints at even larger offsets in the crossline direction add the necessary information in order to reconstruct the 2D-field for a broader offset range. We have not included those datapoints in our simulation because in practice they would fall below the noise floor.

We next create the synthetic aperture source by weighting and combining the recorded fields for a set of source positions in the inline direction. We use the $2 \mathrm{D}$ version of the weighting function given in equation 4.1, which is $f(x)=\exp \left(-\frac{\left(x-x_{\mathrm{Syn}}\right)^{2}}{2 l^{2} / \nu^{2}}\right)$, where the length of the synthetic aperture source $l$ is $5 \mathrm{~km}$ and the empirical factor $\nu$ is set to 5 . We use an efficient implementation of the synthetic aperture source algorithm that multiplies the data in the wavenumber domain with the Fourier transform of the Gaussian weighting function $f(x)$ exploiting the laterally invariant medium. The resul- 

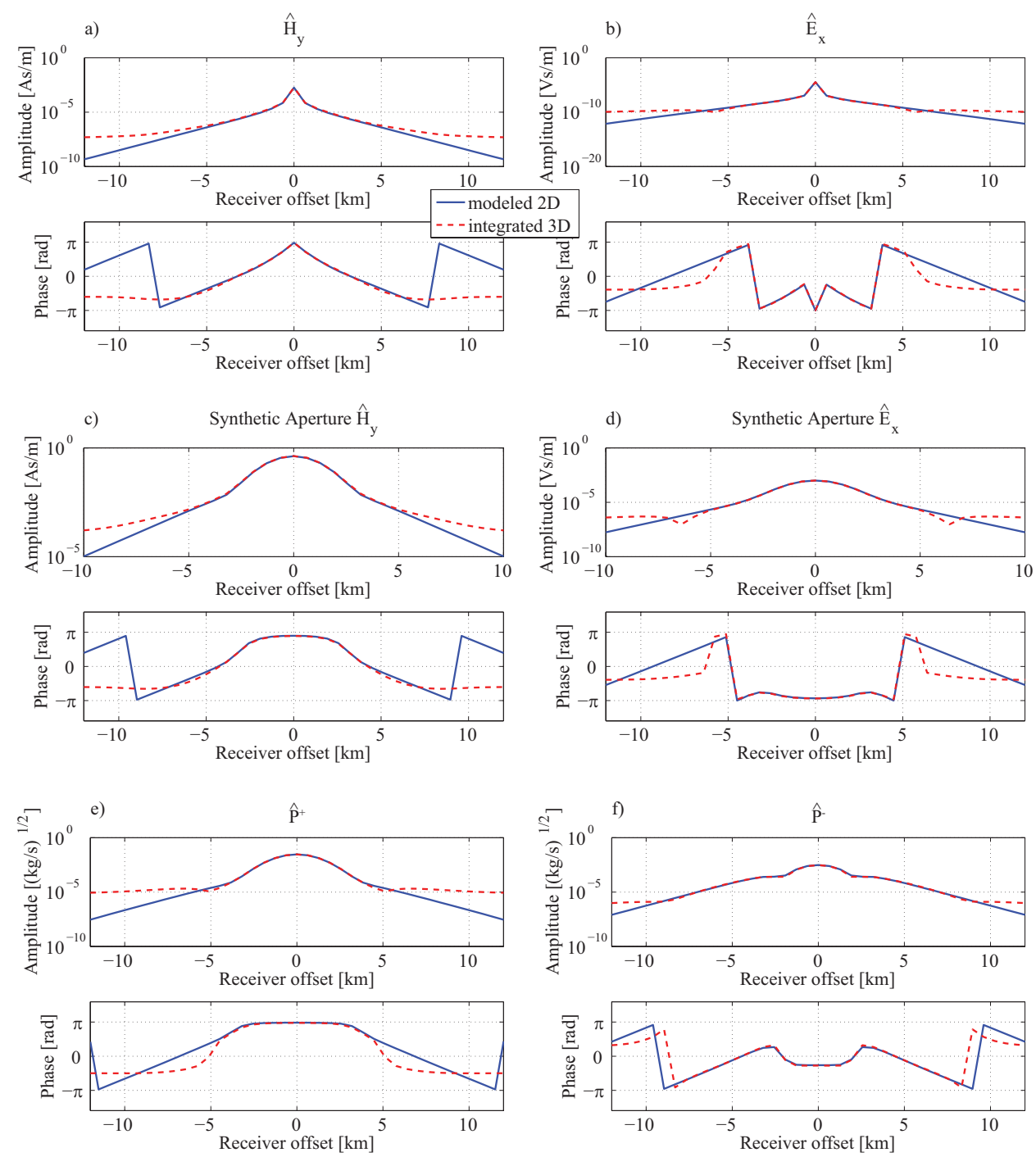

Figure 4.4: The amplitude and the phase of a) $H_{y}$ and b) $E_{x}$ as a function of receiver offset after the integration in the crossline direction. Subfigures c) and d) show the amplitude and the phase of the same electromagnetic fields after the synthetic aperture source in the inline direction has been created. Subfigure e) shows the downward decaying field $P^{+}, f$ ) the upward decaying field $P^{-}$. The solid blue curve is an analytically modelled $2 D$ TM-mode field, the dashed red curve is a $2 D$ TM-mode field computed from a $3 D$ field by integration in the crossline direction. The receiver spacing is $640 \mathrm{~m}$. 
ting electromagnetic fields are shown in Figures 4.4c and 4.4d. Through this procedure the narrow peak of the electromagnetic field around zero offset is turned into a broad and smooth maximum. This smoother curve has a much more limited wavenumber spectrum and can, therefore, be sampled more sparsely. Aliasing of the electromagnetic field can in this way be avoided.

After transforming the electromagnetic fields to the wavenumber-domain, a $2 \mathrm{D}$ decomposition is applied. The $3 \mathrm{D}$ four-by-four decomposition matrix is reduced in $2 \mathrm{D}$ to a two-by-two decomposition matrix as described in section 4.12. The decomposed fields, transformed back to the space-domain, are shown in Figures 4.4e and 4.4f. The error introduced due to incomplete reconstruction of the 2D-field goes for a large part into the downward decaying field $\hat{P}^{+}$, leading to a rather large discrepancy between the dashed red (integrated 3D data) and the solid blue curve (modelled 2D data) at offsets larger than $5 \mathrm{~km}$. In contrast, the upward decaying field $\hat{P}^{-}$is accurately reconstructed almost up to offsets of $10 \mathrm{~km}$. The reason for this asymmetric error propagation lies in the reconstruction of the $2 \mathrm{D}$-field. The integration of the original 3D-field in the crossline direction should have eliminated the airwave, but the reconstruction is not perfect and the airwave remains at least partially in the data dominating offsets larger than $5 \mathrm{~km}$. The largest part of the airwave, the direct airwave, is a downward decaying event and therefore ends up in the downward decaying field after decomposition.

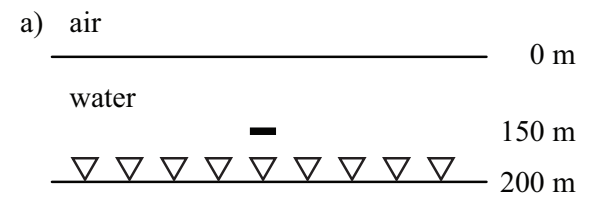

laterally invariant subsurface b) air

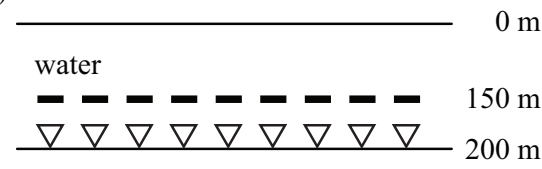

laterally varying subsurface

Figure 4.5: Source-receiver geometry in a) the frequency-wavenumber domain interferometry and b) the frequency-space domain interferometry. Receivers are depicted as white triangles, sources as black horizontal bars.

In the synthesized-2D case, the crossline wavenumber $k_{y}$ and the TEmode part of equations 4.8 and 4.9 can be omitted, leading to

$$
\tilde{P}^{T M-}\left(k_{x}, z_{1}, \omega\right)=\tilde{R}_{0}^{T M}\left(k_{x}, z_{1}, \omega\right) \tilde{P}^{T M+}\left(k_{x}, z_{1}, \omega\right),
$$

where $\tilde{R}_{0}^{T M}$ is the TM-mode reflection response of the subsurface. We can solve for the reflection response by a simple division (for convenience, the function arguments are dropped)

$$
\tilde{R}_{0}^{T M}=\tilde{P}^{T M-}\left(\tilde{P}^{T M+}\right)^{*}\left[\tilde{P}^{T M+}\left(\tilde{P}^{T M+}\right)^{*}+\varepsilon^{2} f_{k}\right]^{-1},
$$


where $\varepsilon$ is a stabilization parameter for the division scaled with a factor $f_{k}$ given by the mean of the absolute value of $\tilde{P}^{T M+}\left(\tilde{P}^{T M+}\right)^{*}$. The superscript * indicates complex conjugation. As mentioned earlier, by solving for the reflection response in the frequency-wavenumber domain, we assume that the medium is laterally invariant. Therefore, only one source position at the centre of the receiver array is necessary (Figure 4.5a). If the medium features strong variations in the inline direction, the deconvolution step needs to be carried out in the frequency-space domain and many source positions are required to solve for the reflection response (Figure 4.5b). Figure 4.6a shows the amplitude and the phase of the reflection response in the frequencywavenumber domain retrieved from equation 4.11. The reflection response retrieved from the modelled 2D-data is plotted in blue whereas the reflection response retrieved from the integrated 3D dataset is shown in red. The excellent match of the two curves in amplitude and phase shows that the artefacts introduced due to incomplete reconstruction of the 2D-field have been suppressed to a large part from the integrated 3D data. The grey curve is the directly modelled reflection response computed on a much denser grid. In other words, the grey curve is the correct response, which we aim to retrieve from the data. In Figure 4.6a, the modelled reflection response fits well with the retrieved reflection responses, within the bandwidth defined by the receiver sampling distance, indicating that the retrieval is indeed correct.

In the frequency-space domain (Figure 4.6b), the blue and the red curve are in strong agreement as well. However, at offsets larger than $5 \mathrm{~km}$ the retrieved reflection response diverges from the directly modelled reflection response. This discrepancy depends on the choice of $\nu$ in the synthetic aperture source, which determines the width of the Gaussian distribution function. Small values of $\nu$ lead to the oscillation in the phase and the overestimation of the amplitude at large offsets visible in Figure 4.6b. These artefacts disappear for large values of $\nu$. However, the choice of $\nu$ depends on the receiver spacing. If a denser receiver spacing is used, fewer of the high wavenumbers need to be filtered out and, therefore, a larger value for $\nu$ can be chosen. Consequently, the oscillation in the phase and the overestimation of the amplitude are indirectly related to the receiver sampling distance, because large receiver sampling distances require small values for $\nu$ in order to sufficiently damp the large wavenumbers in the data. Since the described artefacts distort only data of low amplitude at large offsets, we do not consider it as problematic. 

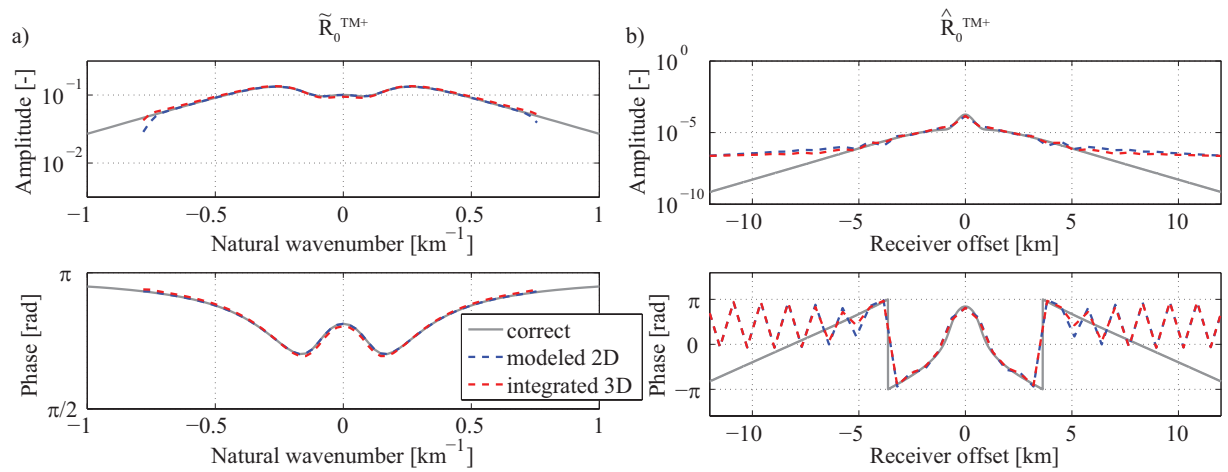

Figure 4.6: Amplitude and phase of the reflection response a) in the frequencywavenumber domain and b) in the frequency-space domain. The solid grey curve is the directly modelled reflection response, in other words, the correct reflection response, the dashed blue curve is the reflection response retrieved from the analytically modelled 2D TM-mode dataset and the dashed red curve is the reflection response retrieved from the 2D TM-mode dataset computed from a $3 D$ field by integration in the crossline direction. The receiver spacing for the blue and red curves is $640 \mathrm{~m}$. The directly modelled reflection response was computed with a receiver spacing of 10 $m$. The natural wavenumber is the radial wavenumber divided by $2 \pi$.

\subsection{Synthesized-2D interferometry in the frequen- cy-space domain}

Interferometry in the frequency-space domain does not pose any limitations on the medium in the inline direction. In other words, the medium does not need to be horizontally layered as required for interferometry in the frequency-wavenumber domain. However, in order to create the synthesized2D data we assumed a laterally invariant medium in the crossline direction. Note, that there is still no TE-mode in the synthesized-2D data because it has been suppressed through the integration across the source lines in the crossline direction. In the frequency-space domain, equation 4.11 becomes a matrix equation

$$
\hat{\mathbf{R}}_{\mathbf{0}}^{\mathbf{T M}}=\hat{\mathbf{P}}^{\mathbf{T M}-}\left(\hat{\mathbf{P}}^{\mathbf{T M}+}\right)^{\dagger}\left[\hat{\mathbf{P}}^{\mathbf{T M}+}\left(\hat{\mathbf{P}}^{\mathbf{T M}+}\right)^{\dagger}+\varepsilon^{2} f_{x} \mathbf{I}\right]^{-1},
$$

where $\hat{\mathbf{P}}^{\mathbf{T M}+}, \hat{\mathbf{P}}^{\mathbf{T M}-}$ and $\hat{\mathbf{R}}_{\mathbf{0}}^{\mathbf{T M}}$ are matrices organized in the same way as in equation 4.5. The superscript $\dagger$ means complex conjugation and matrix transposition. The deconvolution is stabilized by $\varepsilon^{2}$ times the scaling factor $f_{x}$, which is the mean of the absolute value of the diagonal elements of 
$\hat{\mathbf{P}}^{\mathbf{T M}+}\left(\hat{\mathbf{P}}^{\mathbf{T M}+}\right)^{\dagger}$. The matrix $\mathbf{I}$ is the identity matrix. To evaluate equation 4.12, many source positions are required (Figure 4.5b) in contrast to the frequency-wavenumber domain solution which can be computed for just one source position assuming a laterally invariant medium.
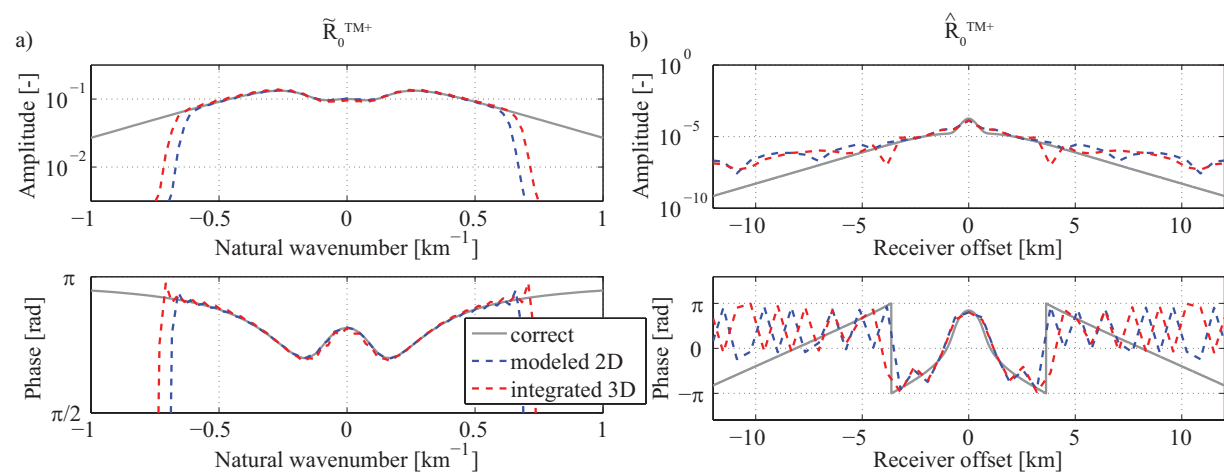

Figure 4.7: Same as Figure 4.6 for the reflection response retrieved in the frequency-space domain instead of the frequency-wavenumber domain.

Figure 4.7 shows the amplitude and the phase of the reflection response retrieved by equation 4.12 in the frequency-space domain. The plot features the reflection response for a redatumed source at the centre of the receiver array in the wavenumber and in the space domain. Note that the wavenumberdomain reflection response (Figure 4.7a) is computed by a Fourier transform of the space-domain reflection response. Equation 4.12 retrieves redatumed sources at all receiver locations in one step, but for a layered medium, they differ only with respect to the location of the redatumed source, because the subsurface is not changing as a function of lateral position. Therefore, the response to only one redatumed source is shown here. The resulting reflection responses are similar to the reflection responses retrieved in the wavenumber domain using equation 4.11, which are shown in Figure 4.6. One significant difference is, that the edges of the wavenumber domain reflection responses vanish (Figure 4.7a). We interpret this as a consequence of the numerical Fourier transform of the reflection response from the space domain, where it is retrieved, to the wavenumber domain. In the space domain, the retrieved reflection responses retrieved via the space domain (Figure 4.7b) are noisier than their counterparts retrieved via the wavenumber domain (Figure 4.6b). These artefacts depend firstly, as mentioned before, on the choice of the parameter $\nu$ and, therefore, on the receiver spacing, because the latter defines the optimal value of $\nu$, which is the largest value that damps the aliased wave- 
numbers at the receiver spacing used. Consequently, smaller receiver spacing distances allow to use larger values of $\nu$ and, therefore, minimize these artefacts. Secondly, the artefacts may also originate from the decomposition. The decomposition, which is carried out per source position, assumes that for each source, enough receivers on both sides of the source are available. This assumption is not fulfilled for sources towards the edge or even outside the receiver array and, therefore, introduces artefacts. A third reason for the noisier retrieval of the reflection response may be found in the difference of the stabilization parameter of equation 4.11 and 4.12 . While in the frequency-wavenumber domain unstable wavenumbers are stabilized, in the frequency-space domain the field at unstable spatial locations is stabilized. The wavenumber-domain stabilization may be closer to the actual physics and, therefore, deliver a less noisy result. Due to all these effects, the result of the space-domain implementation may be closer to the expected result on measured data.

\subsection{Full-3D interferometry in the frequency-wave- number domain}

For full-3D interferometry, data on a grid as sketched in Figure 4.2a and recordings of all four horizontal electromagnetic field components are necessary for the decomposition into upward and downward decaying fields. Figure 4.8 shows the four horizontal components of the electromagnetic field on a grid after the synthetic aperture source is created using the weighting function of equation 4.1. Similar to the synthesized-2D case, the length of the synthetic aperture source $l$ is chosen $5 \mathrm{~km}$ and the parameter $\nu$ equal to 5 . We also implement the synthetic aperture source in the wavenumber domain for efficiency reasons. The source is oriented in the inline direction and the receiver spacing distance is $640 \mathrm{~m}$ in both horizontal directions. For creating the synthetic aperture source, we assumed to have a similar dense source coverage in the inline and the crossline directions. In practice, the crossline source sampling is sparser than the inline source sampling. The effects of sparser source sampling in the crossline direction remain to be investigated, but are beyond the scope of this chapter. At zero inline offset or at zero crossline offset relative to the source position, the amplitude of $E_{y}$ and $H_{x}$ vanishes because of symmetry reasons. Due to the relatively sparse sampling, this zero amplitude shows up as thick blue lines in Figures 4.8b and 4.8c.

Since this is a 3D dataset, we do not only retrieve the TM-mode reflection response, but also the TE-mode reflection response as well as cross-mode re- 
a)

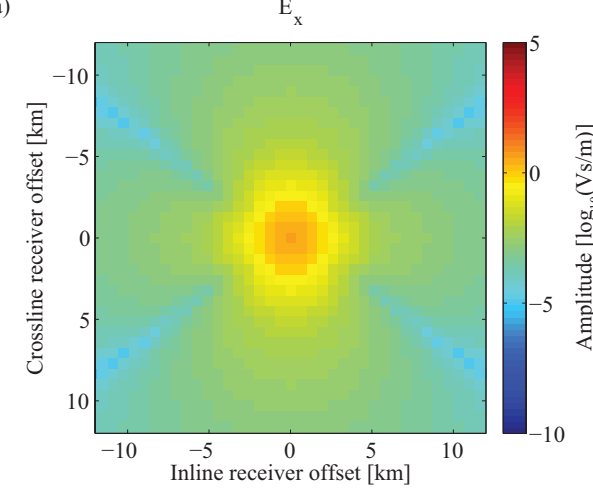

c)

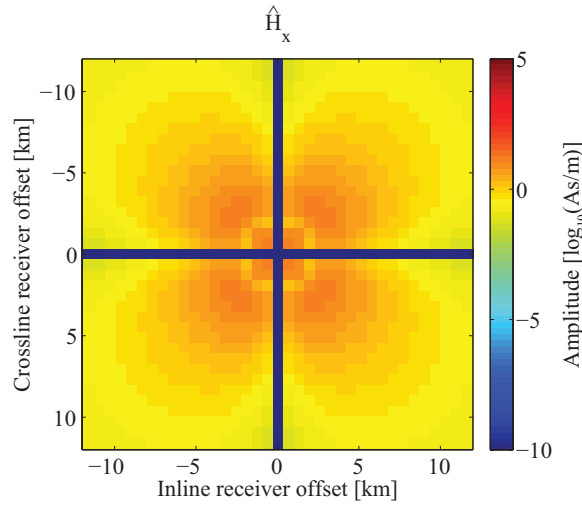

b)

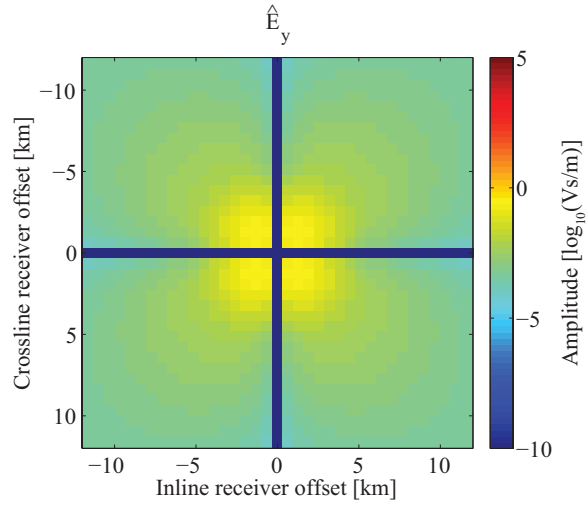

d)

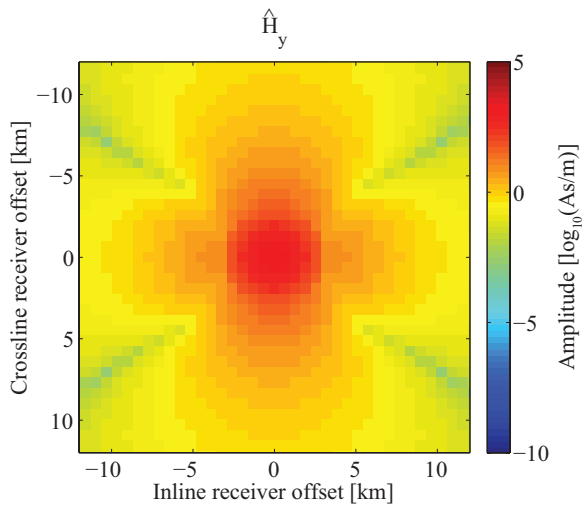

Figure 4.8: The four horizontal components of the electromagnetic field in the frequency-space domain for a receiver spacing of $640 \mathrm{~m}$ after the synthetic aperture source has been created: a) $E_{x}$, b) $E_{y}$, c) $H_{x}$ and d) $H_{y}$.

flection responses. If the medium contained lateral variations, the cross-mode reflection responses would be non-zero. In the frequency-wavenumber domain implementation, where we assume a layered medium, the cross-terms can be used as a quality check. If they do not vanish, most probably the assumption of a horizontally layered medium is violated and the frequency-wavenumber domain solution is not appropriate. After Fourier-transforming the data presented in Figure 4.8 to the frequency-wavenumber domain and decomposing the fields in upward and downward decaying fields, the most straightforward approach to retrieve the reflection response is by evaluating equation 4.11 separately for each mode on a grid instead of on a line of wavenumbers. Unfortunately, the illumination from one source component (inline) is insufficient for a proper retrieval of the 3D multicomponent reflection response. Furthermore, if one aims to retrieve the cross-mode reflection responses for 
a quality check, an independent treatment of each mode is not possible.

In this study, we overcome this limitation by including data of the four horizontal electromagnetic field components generated by a source in the crossline direction. Furthermore, we use a formulation that couples all modes instead of dealing with them independently in order to retrieve the cross-mode reflection responses, which are supposed to be zero. The forward problem (equation 4.5) becomes then in the frequency-wavenumber domain

$$
\left(\begin{array}{cc}
\tilde{P}_{\mathrm{X}-\mathrm{src}}^{T M,-} & \tilde{P}_{\mathrm{y}-\mathrm{src}}^{T M,-} \\
\tilde{P}_{\mathrm{X}-\mathrm{src}}^{T E,-} & \tilde{P}_{\mathrm{y} \text {-src }}^{T E,-}
\end{array}\right)=\left(\begin{array}{cc}
\tilde{R}_{0}^{T M, T M} & \tilde{R}_{0}^{T M, T E} \\
\tilde{R}_{0}^{T E, T M} & \tilde{R}_{0}^{T E, T E}
\end{array}\right)\left(\begin{array}{ccc}
\tilde{P}_{\mathrm{X} \text {-src }}^{T M,+} & \tilde{P}_{\mathrm{y} \text {-src }}^{T M,+} \\
\tilde{P}_{\mathrm{X} \text {-src }}^{T E} & \tilde{P}_{\mathrm{y} \text {-src }}^{T E,+}
\end{array}\right),
$$

where the subscripts $\mathrm{x}$-src and $\mathrm{y}$-src in the decomposed fields define the inline and crossline orientation of the source, respectively. All other superscripts and subscripts are the same as before.

Equation 4.13 can be solved for the matrix of reflection responses for each wavenumber separately by inverting the two-by-two matrix of the downward decaying field

$$
\begin{aligned}
& \left(\begin{array}{ll}
\tilde{R}_{0}^{T M, T M} & \tilde{R}_{0}^{T M, T E} \\
\tilde{R}_{0}^{T E, T M} & \tilde{R}_{0}^{T E, T E}
\end{array}\right)= \\
& \left(\begin{array}{cc}
\tilde{P}_{\mathrm{X}-\mathrm{src}}^{T M,-} & \tilde{P}_{\mathrm{y}-\mathrm{src}}^{T M,-} \\
\tilde{P}_{\mathrm{X}-\mathrm{src}}^{T E} & \tilde{P}_{\mathrm{y}-\mathrm{src}}^{T E,-}
\end{array}\right)\left[\left(\begin{array}{cc}
\tilde{P}_{\mathrm{X}-\mathrm{src}}^{T M,+} & \tilde{P}_{\mathrm{y}-\mathrm{src}}^{T M,+} \\
\tilde{P}_{\mathrm{X} \text {-src }}^{T E,+} & \tilde{P}_{\mathrm{y} \text {-src }}^{T E,+}
\end{array}\right)+\varepsilon^{2}\left(\begin{array}{ll}
1 & 0 \\
0 & 1
\end{array}\right)\right]^{-1},
\end{aligned}
$$

where $\varepsilon$ is a stabilization parameter similar to the one in equations 4.11 and 4.12. The stabilization parameter is in this case not scaled with a factor $f$ because the solution is much less dependent on the stabilization parameter than in equations 4.11 and 4.12. Although it would be possible to choose a different stabilization parameter for each wavenumber, we use the same for the complete wavenumber spectrum. In this case, we do not use the least-squares formulation shown in the previous sections because we have a square two-by-two matrix. Note that as for the synthesized-2D interferometry in the frequency-wavenumber domain, also the full-3D interferometry in the frequency-wavenumber domain requires the medium to be laterally invariant. The benefit is that also in this case we require only one source location (Figure 4.5a). For any general medium, full-3D interferometry with several source locations needs to be applied in the frequency-space domain, which is discussed in the next section.

The reflection responses retrieved with equation 4.14 miss one datapoint at $k_{x}=k_{y}=0$, i.e., the reflection response for vertical incidence, because that wavenumber component of the field is not excited by a finite source. In order 

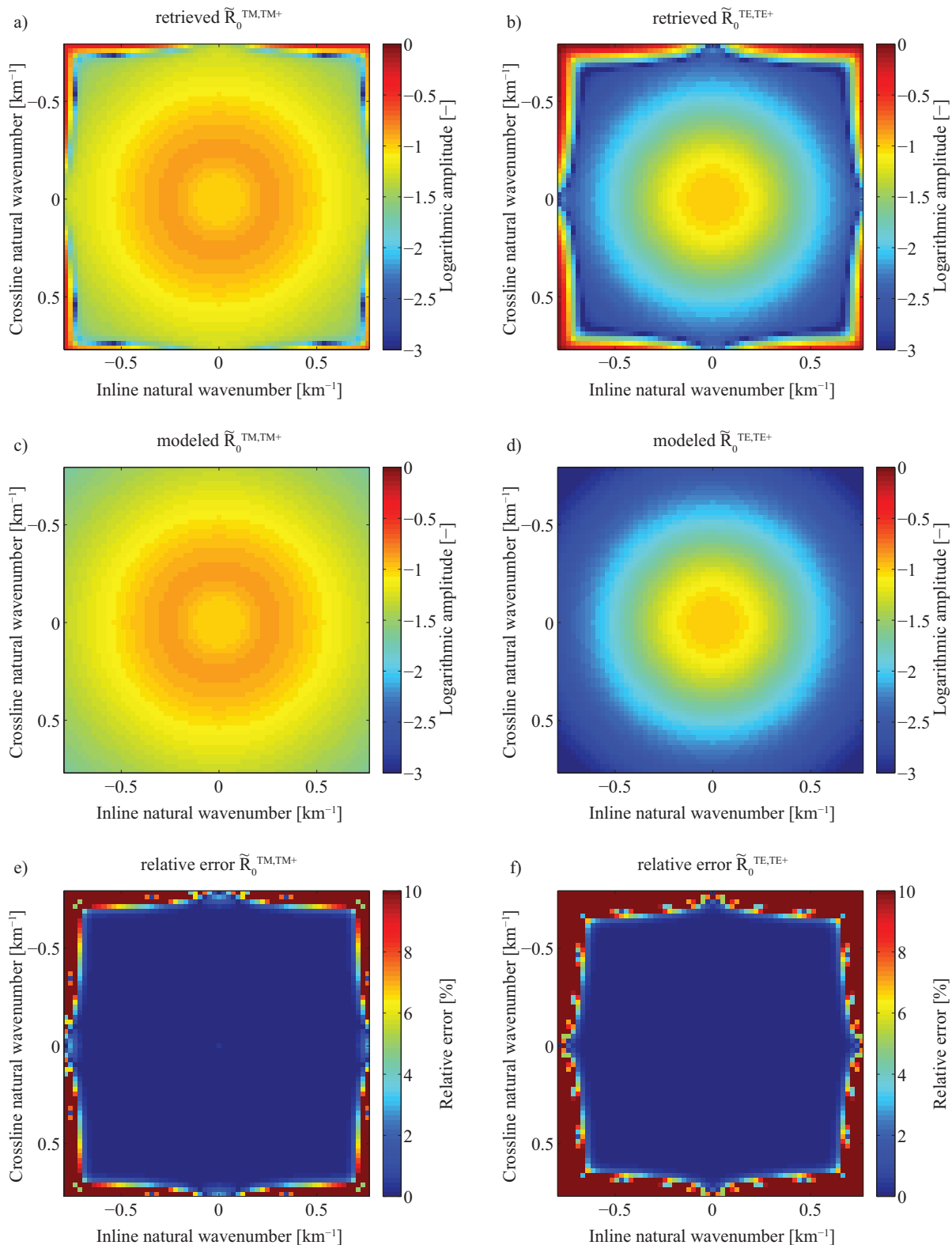

Figure 4.9: The reflection responses retrieved and plotted in the frequencywavenumber domain: a) retrieved TM-mode, b) retrieved TE-mode, c) modelled TM-mode, d) modelled TE-mode, e) relative error TM-mode and $f$ ) relative error TE-mode. 
to fill the gap, this one point is given the value of its neighbouring point, i.e., the smallest wavenumber available in the $k_{x}$-direction. The amplitude of the resulting retrieved reflection responses in the frequency-wavenumber domain for the TM-mode and the TE-mode are shown in Figures 4.9a and 4.9b. The complete wavenumber range available for a receiver-sampling distance of 640 $\mathrm{m}$ is shown. The retrieved reflection responses are radially symmetric and agree reasonably well compared with the directly modelled reflection responses shown in Figures 4.9c and 4.9d, except for edge artefacts that disturb the signal at the largest wavenumbers. These artefacts can be damped by using a larger stabilization parameter at these wavenumbers, but the improvement is only cosmetic, because the retrieved reflection response for these wavenumbers is simply minimized without producing the correct values. The relative error between retrieved and modelled reflection responses is shown in Figures $4.9 \mathrm{e}$ and $4.9 \mathrm{f}$. In the TM-mode as well as in the TE-mode, the relative error never exceeds $3 \%$ except at high wavenumbers (i.e., $>0.65 \mathrm{~km}^{-1}$ for the TM-mode and $>0.6 \mathrm{~km}^{-1}$ for the TE-mode). The reflection responses for mode conversions are correctly retrieved to be numerically zero (not shown), i.e., the mean of the cross-mode reflection responses is more than three orders of magnitude smaller than the mean of the TM-mode reflection response (excluding the edge artefacts). This also indicates a proper retrieval of the multicomponent reflection response.

Before the Fourier transform to the frequency-space domain is carried out, a taper is applied to damp the unstable signal close to the Nyquist wavenumbers. The retrieved and modelled reflection responses in the frequency-space domain are shown in Figures 4.10a to 4.10d. Note that the modelled reflection response has been bandlimited to the same bandwidth as the reflection response retrieved with a receiver spacing of $640 \mathrm{~m}$. The corresponding relative error plots are shown in Figures 4.10e and 4.10f. The reflection responses are retrieved well, apart from instabilities confined to the zero inline and zero crossline offset. These artefacts are introduced in the Fourier transform because the reflection response is aliased at a receiver spacing of $640 \mathrm{~m}$. The same artefacts appear also in the bandlimited modelled reflection response, which confirms that aliasing is the cause of the artefacts. Only a denser receiver spacing will avoid these artefacts. In the diagonal direction $(x= \pm y)$ no aliasing takes place due to the larger radial wavenumber range. Since in a layered medium the reflection responses are radial functions, no information is lost in that case. The relative error plots show errors smaller than $10 \%$ for radial distances from the source smaller than $10 \mathrm{~km}$. At larger radial distances, the relative error increases beyond 10\%, but at those offsets, the signal has decayed already over four orders of magnitude and is, the- 

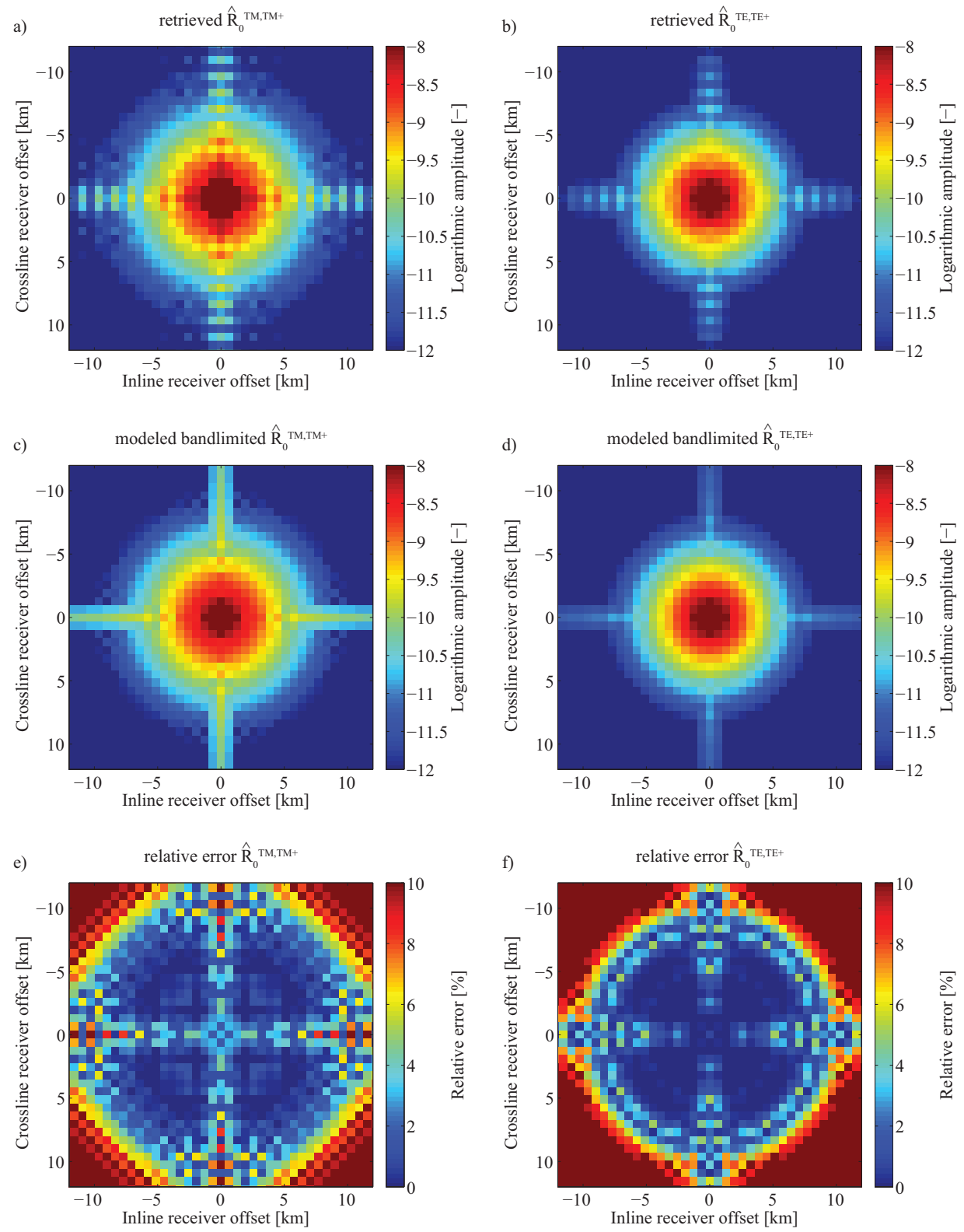

Figure 4.10: Same data as in Figure 4.9 but plotted in the frequency-space domain. 
refore, rather small. Due to the small amplitude of the field at this offset range, even very small absolute errors can show up on the relative error plots with a huge magnitude. Therefore, we neglect these errors. Considering the large receiver-sampling distances used, this retrieval is considered very good. Smaller receiver-sampling distances will lead to an even better retrieval.

\subsection{Full-3D interferometry in the frequency-space domain}

For frequency-space domain full-3D interferometry, no limitations about the medium apply, but, as for the synthesized-2D interferometry in the frequencyspace domain, a set of sources is required to retrieve the reflection response (Figure 4.5b) and not just one as for the frequency-wavenumber domain interferometry. In order to perform 3D-interferometry by MDD in the frequencyspace domain, we rewrite equation 4.13 such that the elements of the matrices become matrices themselves:

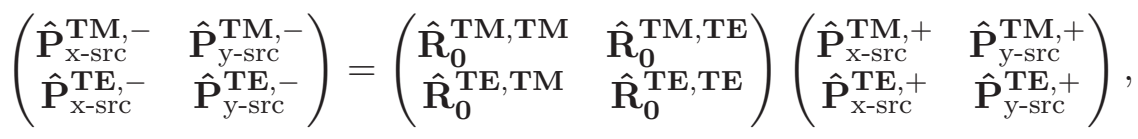

where the matrices $\hat{\mathbf{P}}$ contain the decomposed fields for all receiver positions for one source position along their columns and vice versa for the rows. The matrices $\hat{\mathbf{R}}$ are structured in a similar way, but with the source positions replaced by redatumed source positions. Since at every receiver position a source is redatumed, the matrices $\hat{\mathbf{R}}$ are square. Note that the grid of receivers and the grid of sources is represented in one column or one row, respectively, in order to avoid tensors of higher order than 2 (Kinneging et al., 1989). The disadvantage of this approach is that the matrices are large. For example, for a receiver grid of 64 times 64 receivers, as it is used in this example, one row of the matrix $\hat{\mathbf{P}}_{\mathrm{X} \text {-src }}^{\mathrm{TM},+}$ contains $64^{2}=4096$ elements. To create a similar source coverage as is simulated by solving equation 4.13 in the frequency-wavenumber domain, a grid of $(2 \cdot 64-1)^{2}=$ 16129 sources is necessary. Consequently, matrix $\hat{\mathbf{P}}_{\mathrm{X}-\mathrm{src}}^{\mathrm{TM},+}$ ends up having 4096 times 16129 elements. If the complex data are stored as 64 bit floating point digits, approximately one Gigabyte of memory is required to set up the matrix $\hat{\mathbf{P}}_{\mathrm{X}-\mathrm{src}}^{\mathbf{T M},+}$. Four times more is necessary to keep the complete downward decaying field in memory. To have a slightly more realistic example, we omit source positions far away from the receiver grid. In total, 7744 sources are included in our example dataset. 
We use the following least-squares equation to retrieve the reflection response

$$
\begin{aligned}
& \left(\begin{array}{cc}
\hat{\mathbf{R}}_{0}^{\mathrm{TM}}, \mathbf{T M} & \hat{\mathbf{R}}_{\mathbf{T}}^{\mathrm{TM}, \mathrm{TE}} \\
\hat{\mathbf{R}}_{\mathbf{0}}^{\mathrm{TE}, \mathbf{T M}} & \hat{\mathbf{R}}_{\mathbf{0}}^{\mathrm{TE}, \mathbf{T E}}
\end{array}\right)=
\end{aligned}
$$

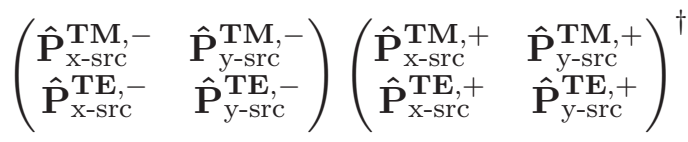

$$
\begin{aligned}
& {\left[\left(\begin{array}{cc}
\hat{\mathbf{P}}_{\mathrm{x} \text {-src }}^{\mathbf{T M},+} & \hat{\mathbf{P}}_{\mathrm{y} \text {-src }}^{\mathbf{T M},+} \\
\hat{\mathbf{P}}_{\mathrm{X}-\mathrm{src}}^{\mathbf{T E},+} & \hat{\mathbf{P}}_{\mathrm{y}-\mathrm{src}}^{\mathbf{T E},+}
\end{array}\right)\left(\begin{array}{cc}
\hat{\mathbf{P}}_{\mathrm{X}-\mathrm{src}}^{\mathbf{T M},+} & \hat{\mathbf{P}}_{\mathrm{y} \text {-src }}^{\mathbf{T M},+} \\
\hat{\mathbf{P}}_{\mathrm{X} \text {-src }}^{\mathbf{T E},+} & \hat{\mathbf{P}}_{\mathrm{y} \text {-src }}^{\mathbf{T E},+}
\end{array}\right)^{\dagger}+\varepsilon^{2} f_{x} \mathbf{I}\right]^{-1} \text {. }}
\end{aligned}
$$

As in the synthesized-2D interferometry in the frequency-space domain, the scaling factor $f_{x}$ is the mean of the absolute value of the diagonal elements of the cross-correlation of the downward decaying field with itself. All other quantities are as defined previously.

The retrieved reflection responses are shown in Figures 4.11a and 4.11b, the directly modelled bandlimited reflection responses are reproduced from Figure 4.10 in Figures 4.11c and 4.11d and the relative error between the two is shown in Figures 4.11e and 4.11f. The retrieval is quite accurate with a relative error smaller than $10 \%$ in most of the large amplitude area. The cross-mode reflection responses show some signal around zero-offset, but it is more than two orders of magnitude smaller than the signal of the TM-mode reflection response at the same location. Therefore, we consider the crossmode reflection responses, also in this case, properly retrieved as numerically zero (not shown). The solution depends strongly on the stabilization parameter $\varepsilon$. Since the retrieval of the reflection response in the frequency-space domain is much more expensive than in the frequency-wavenumber domain, only a limited amount of values for the stabilization parameter could be tested. A better choice may lead to a more accurate solution with a smaller relative error. Especially the TM-mode could be improved. Still, as in the synthesized-2D case, the frequency-space domain solution is slightly worse than the frequency-wavenumber solution. As described above, these artefacts may be caused in the decomposition by improperly sampled fields for sources close to the edge of the receiver array. Note that this algorithm can be applied to any medium although the example shown here is a layered earth situation.

As an alternative to the least-squares solution, a conjugate-gradient scheme may be considered. This would be a way to work around the strong dependency on the stabilization parameter. Furthermore, a conjugate-gradient scheme can be implemented in such a way, that not the complete matrix to 

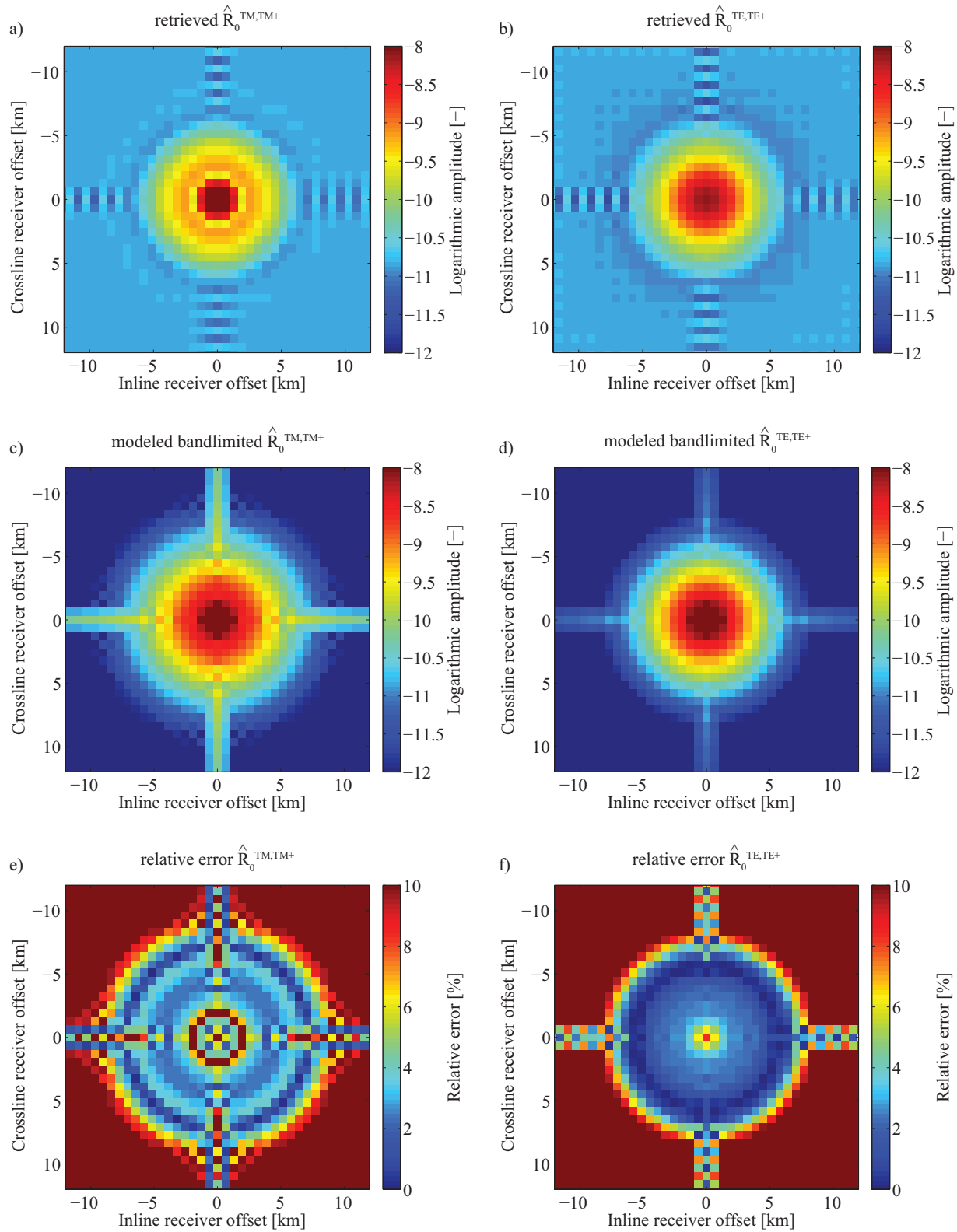

Figure 4.11: The reflection responses retrieved and plotted in the frequency-space domain: a) retrieved TM-mode, b) retrieved TE-mode, c) modelled TM-mode, d) modelled TE-mode, e) relative error TM-mode and f) relative error TE-mode. 
invert needs to be kept in memory. Each iteration can be divided into steps that just require one row and one column of that matrix. One iteration is completed, once all the rows and columns have been processed. This allows to process the current acquisition size on a smaller computer or use even a larger acquisition grid. Furthermore, this algorithm is suited for parallel computing.

\subsection{Reservoir detectability after full-3D interfero- metry in the frequency-wavenumber domain}

As mentioned earlier, the retrieved reflection response suppresses the direct field and the airwave. Therefore, the reservoir should be better detectable. In order to investigate this, we computed the normalized amplitude of the original electromagnetic fields as well as of the reflection responses retrieved in the frequency-wavenumber domain. Normalized amplitude means that the amplitude of a dataset is normalized with a corresponding dataset for a situation without a reservoir, i.e., Figure 4.1 without the $50 \mathrm{~m}$ thick layer of $0.02 \mathrm{~S} / \mathrm{m}$ conductivity at $1200 \mathrm{~m}$ depth.

The normalized amplitude of the four horizontal electromagnetic fields for an inline source as well as the TM-mode and the TE-mode reflection responses are shown in Figure 4.12. A normalized amplitude of around one means that the reservoir cannot or can only barely be seen. Anything significantly larger than one represents a strong imprint of the reservoir. Note that the colour scale is cut at normalized amplitudes of four. The normalized amplitude of the four horizontal electromagnetic field components are at most offsets only slightly above one, indicating that the reservoir leaves only a small imprint in the data. However, in $E_{x}$ and $H_{y}$ (Figures 4.12a and 4.12d) some datapoints show a normalized amplitude up to 12. (Note that amplitudes are clipped at a value of 4 in Figure 4.12. Values larger than 4 are displayed in dark red.) These datapoints lie on or close to zero-crossings of the field (compare with Figures 4.3 and 4.8). Therefore, we consider these large normalized amplitudes to be numerical artefacts.

In contrast, the TM-mode reflection response (Figure 4.12e) shows a normalized amplitude larger than two at all offsets. Over a large area, the normalized amplitude is even larger than four. The best detectability comes from receivers on diagonal locations on the receiver grid because those receivers suffer the least from aliasing artefacts shown in Figure 4.10. Figure 4.12e indicates a strong imprint of the reservoir in the TM-mode reflection response. The situation is completely different for the TE-mode reflection 

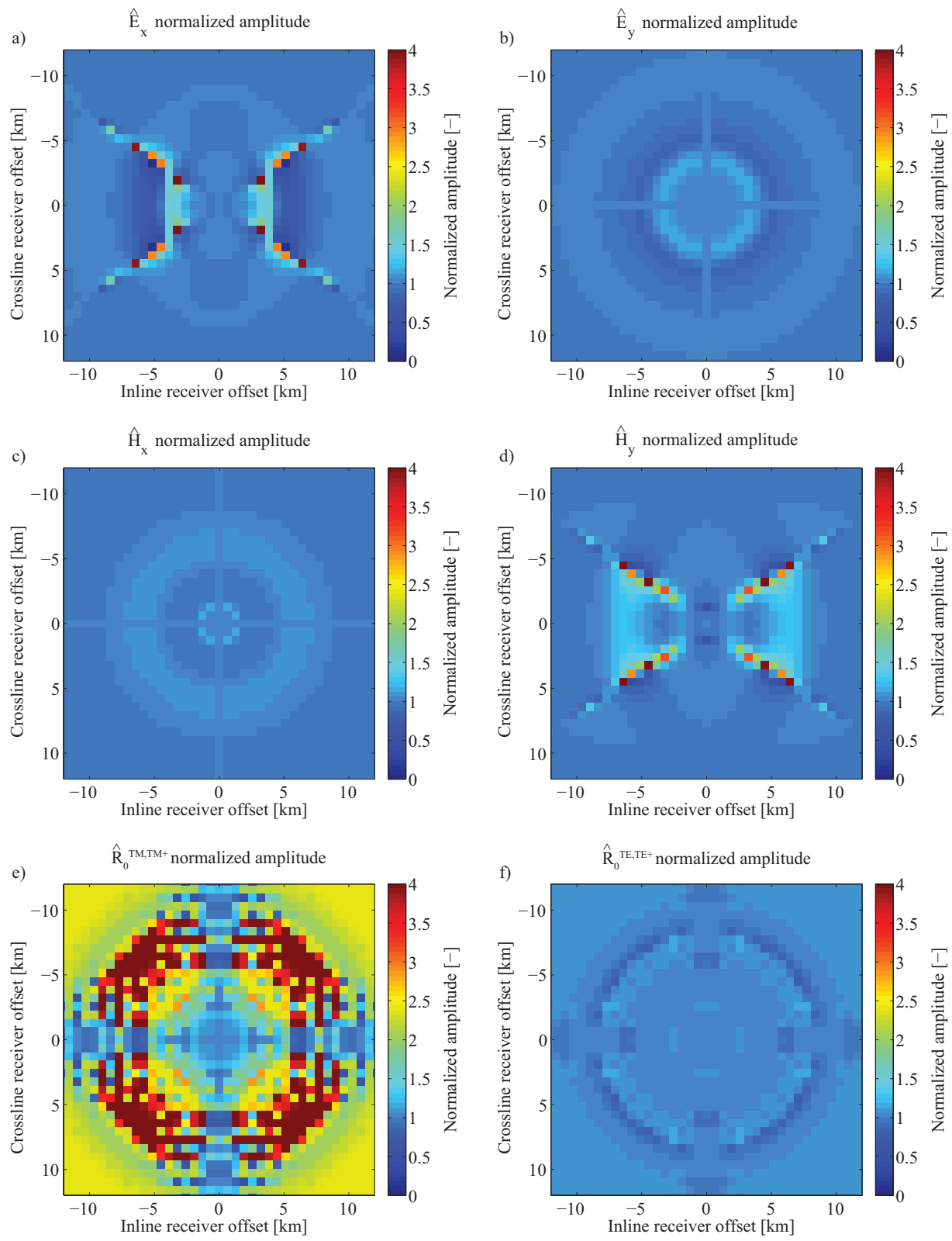

Figure 4.12: Reservoir response, i.e., the amplitude of the data normalized with the amplitude of the same dataset without a reservoir, for a) $\left.E_{x}, b\right) E_{y}$, c) $H_{x}, d$ ) $H_{y}$, e) $R_{0}^{T M, T M}$ and f) $R_{0}^{T E, T E}$. 
response (Figure 4.12f) which shows similar normalized amplitude values as the original electromagnetic fields. Consequently, the TM-mode is much more sensitive to the reservoir than the TE-mode. This is a well known fact described for example by Eidesmo et al. (2002).

\subsection{Parameter dependency of full-3D interferome- try in the frequency-wavenumber domain}

When applying interferometry, the choice of two parameters is crucial. These parameters are, firstly, the conductivity $\sigma$ of the subsurface at the receiver level and, secondly, the stabilization parameter $\varepsilon$. In synthetic examples, we know the conductivity $\sigma$ of the seabed where the receivers are located. In reality, one may have some local measurements or one needs to estimate the conductivity, hence the conductivity value $\sigma$ used for the decomposition will be imprecise. To choose the stabilization parameter $\varepsilon$ for the examples shown in this chapter we used an algorithm which minimizes the relative error between the retrieved and the modelled reflection response. In reality, there is no modelled reflection response and, therefore, $\varepsilon$ needs to be chosen by trial and error, making it unlikely to find the optimum value. A way to find a good value for $\varepsilon$ is to decrease it until the solution gets unstable. A good choice would be the smallest $\varepsilon$ which gives a stable solution. Still, it is important to know the influence of wrongly chosen or imprecise $\varepsilon$ and $\sigma$ on the retrieved reflection response. Inversions based on conjugate gradient schemes or singular value decomposition may be used as an alternative to the least-squares inversion presented here in order to avoid a difficult process to find the proper stabilization parameter. Comparable parameters used in these schemes are closer related to the physics of the problem.

We retrieved reflection responses for a range of conductivity values $\sigma$ and stabilization parameters $\varepsilon$ using full-3D interferometry in the frequencywavenumber domain (equation 4.14). For each of these retrieved reflection responses we computed the cumulative relative error $E$ for each mode independently:

$$
E^{m, n}(\sigma, \varepsilon)=\frac{\Sigma_{\text {samples }}|| \tilde{R}_{0, \text { ret }}^{m, n}(\sigma, \varepsilon)|-| \tilde{R}_{0, \text { mod }}^{m, n} \|}{\Sigma_{\text {samples }}\left|\tilde{R}_{0, \text { mod }}^{m, n}\right|},
$$

where the superscripts $m$ and $n$ indicate the mode of the receiver and the mode of the redatumed source, respectively. In this study, we only consider situations where $m=n$, because the cross-terms $(m \neq n)$ vanish in a horizontally layered medium. The subscripts "ret" and "mod" refer to retrieved 
and modelled reflection response, respectively. The sum is taken over a range of samples in the frequency-wavenumber domain. We do not include wavenumbers close to the Nyquist frequency $\left(>0.6 \mathrm{~km}^{-1}\right)$ because they show unreasonably high amplitudes for a vast range of small $\varepsilon$, which would dominate the cumulative relative error function although the retrieval of the reflection response is quite good otherwise.
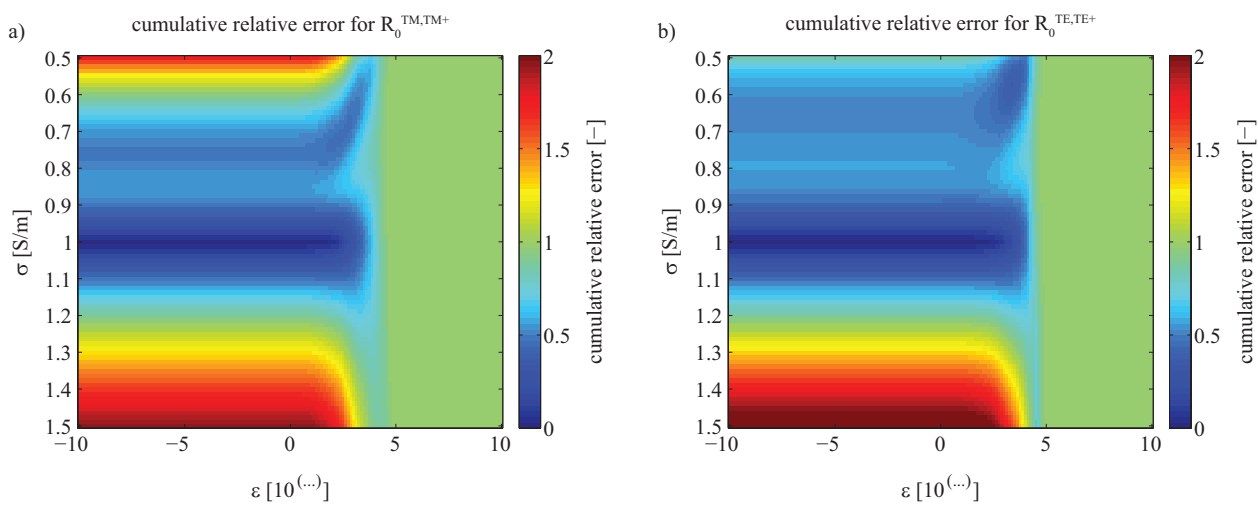

Figure 4.13: The cumulative error function for different conductivity values $\sigma$ and different stabilization values $\varepsilon$ for a) the TM-mode and b) the TE-mode reflection response.

Figure 4.13 shows the cumulative error for the TM-mode and TE-mode situation whose retrieved reflection responses for the optimal choice of $\sigma$ and $\varepsilon$ are shown in Figures 4.9 and 4.10. If the cumulative relative error is close to zero (dark blue areas) the reflection response is retrieved well and if the cumulative relative error is large (red areas), the retrieved reflection response is far from the correct solution. The correct conductivity $\sigma$ is in our situation $1 \mathrm{~S} / \mathrm{m}$. For both modes, we find an area between $0.9 \mathrm{~S} / \mathrm{m}$ and $1.1 \mathrm{~S} / \mathrm{m}$ which is dark blue. In other words, the conductivity needs to be known with a precision of $\pm 0.1 \mathrm{~S} / \mathrm{m}$ of the correct value in our simulation. The plots in Figure 4.13 are asymmetric relative to the conductivity axis, which indicates that overestimating the conductivity leads to a larger error than underestimating it.

The stabilization parameter $\varepsilon$ hardly affects the solution as long as it is chosen small enough, i.e., smaller than approximately $10^{2}$. Too large values of $\varepsilon$ on the other hand lead to a very small amplitude of the retrieved reflection response and, consequently, the relative cumulative error becomes one (green areas). While interferometry in 3D in the frequency-wavenumber domain is stable, full-3D interferometry in the frequency-space domain as well as 
the synthesized-2D cases tend to become unstable for small values of $\varepsilon$ (not shown).

\subsection{Discussion}

Synthesized-2D interferometry has the advantage that only one line of receivers is necessary and not a complete grid as for the full-3D interferometry. On the other hand, it requires a set of source positions off this receiver line in order to create a virtually infinitely long source by integration in crossline direction to make 2D processing of 3D data possible. Our numerical experiments have shown that in our case 35 source positions are sufficient. These source positions are densely spaced close to the receiver line and sparser spaced far away from the receiver line. Since we determined the positions of the source lines by trial and error, we believe that an optimization routine would find a distribution of fewer source lines. Another way to reduce the number of source lines is to acquire several source lines in one sailing line exploiting the dense source line spacing close to the receiver line. Consequently, the required amount of boat lines can be significantly reduced in practice. Since this procedure assumes no variations of the medium in the crossline direction, the amount of source lines can be cut in half by just acquiring source lines on one side of the receiver line and then doubling the measurements. On the other hand, data acquired from sources located on both sides of the receiver line can serve as a quality check. When creating the 2D-data from the 3D-data by integration, the $E_{y}$ and $H_{x}$ components should vanish. If these two components are not close to zero after the integration, the medium features strong variations in crossline direction or the source lines are not chosen properly. Consequently, the 2D-data will be far from correct.

In the synthesized-2D case, solving for the reflection response in the frequency-wavenumber domain instead of in the frequency-space domain reduces the problem to a scalar division instead of a matrix inversion. In the full-3D case, solving for the reflection response in the frequency-wavenumber domain still involves a matrix inversion because all four components of the reflection response are retrieved. In the following, we derive a set of two scalar equations to retrieve the reflection response in the frequency-wavenumber domain by setting the cross-terms $\tilde{R}_{0}^{T M, T E}$ and $\tilde{R}_{0}^{T E, T M}$ to zero. We start with equation 4.13 and multiply both sides with the complex conjugate and transposed matrix containing the downward decaying field. This gives for 
the TM-mode and the TE-mode reflection response:

$$
\begin{aligned}
& \tilde{R}_{0}^{T M, T M}= \frac{\tilde{P}_{\mathrm{x} \text {-src }}^{T M,-} \tilde{P}_{\mathrm{X} \text {-src }}^{T M,+*}+\tilde{P}_{\mathrm{y}-\mathrm{src}}^{T M,-} \tilde{P}_{\mathrm{y}-\mathrm{src}}^{T M,+*}}{\tilde{P}_{\mathrm{X} \text {-src }}^{T M,+} \tilde{P}_{\mathrm{X}-\mathrm{src}}^{T M,+*}+\tilde{P}_{\mathrm{y}-\mathrm{src}}^{T M,+} \tilde{P}_{\mathrm{y}-\mathrm{src}}^{T M,+*}}, \\
& \tilde{R}_{0}^{T E, T E}=\frac{\tilde{P}_{\mathrm{X} \text {-src }}^{T E,-} \tilde{P}_{\mathrm{X}-\mathrm{src}}^{T E,+*}+\tilde{P}_{\mathrm{y}-\mathrm{src}}^{T E,-} \tilde{P}_{\mathrm{y}-\mathrm{src}}^{T E,+*}}{\tilde{P}_{\mathrm{X} \text {-src }}^{T E,+} \tilde{P}_{\mathrm{X}-\mathrm{src}}^{T E,+*}+\tilde{P}_{\mathrm{y}-\mathrm{src}}^{T E,+} \tilde{P}_{\mathrm{y}-\mathrm{src}}^{T E,+*}},
\end{aligned}
$$

where the superscript star $*$ means complex conjugation. Consequently, also in the full-3D case in the frequency-wavenumber domain the reflection response can be retrieved without performing a matrix inversion. Advantages of carrying out the matrix inversion are that in one step the TM-mode as well as the TE-mode reflection response are retrieved and, as mentioned before, the conversions can be used as a quality check.

All the examples shown here are based on noise-free data. Random noise as well as random errors in receiver position and orientation for 2Dinterferometry are discussed by Hunziker et al. (2012). That study showed that even relatively large levels of random noise poses no problem because it averages out when the synthetic aperture source is created. Random positioning and orientation errors limit the bandwidth of the properly retrieved reflection response. Only rather large receiver positioning errors (up to 50 $\mathrm{m}$ ) but already small orientation errors (up to $10^{\circ}$ ) disturb the data significantly. Consequently, receiver positioning errors pose no problem either. Receiver orientation errors can be avoided by using six component receivers and measuring the orientation of the receivers. Subsequently, the data can be rotated virtually in order to overcome orientation errors. We assume that in $3 \mathrm{D}$ random noise and random errors in receiver position and orientation affect the data in a similar way as in $2 \mathrm{D}$.

\subsection{Conclusions}

Interferometry by MDD applied to CSEM data suppresses any interactions of the signal with the water and the air-water interface (including the airwave). Furthermore, the direct field is suppressed and the source is redatumed to the receiver level. Since interferometry by MDD is a data-driven method, no information about the subsurface or the source position are required except the material parameters of the seabed and the receiver positions and orientations. We applied interferometry by MDD to synthesized-2D and full$3 \mathrm{D}$ synthetic data in the frequency-wavenumber and in the frequency-space domain. 
We have shown that synthesized-2D interferometry is able to retrieve the reflection response well, but only for a limited offset range in the inline direction. For large receiver spacings, the parameter $\nu$, which determines the width of the Gaussian function used for weighting the various source positions for the synthetic aperture source, has to be chosen small in order to suppress the high-wavenumber signal. This leads to artefacts in the retrieved reflection response in the space domain at large offsets. Synthesized-2D interferometry applied in the wavenumber domain is computationally very efficient, because the deconvolution process is reduced to a scalar division. A disadvantage is that it assumes the subsurface to be laterally invariant. That also means that only one source location is required. In contrast, synthesized-2D interferometry in the space domain can be applied to a medium featuring any variations in the inline direction while being constant in the crossline direction, but requires several source positions. A disadvantage is that the matrix inversion is slightly more computationally intensive than the scalar division, but in $2 \mathrm{D}$ the difference in computation time between the two algorithms is not so large because the matrices are small.

Considering computational efficiency, the difference of solving for the reflection response in the wavenumber or in the space domain is more pronounced in full-3D interferometry. While the process takes only a few minutes in the wavenumber domain, it takes up to several days in the space domain (including synthetic aperture and decomposition of all the source positions required). As in the synthesized-2D case, the wavenumber-domain solution requires the medium to be laterally invariant, whereas the frequency-space domain algorithm presented here can be applied to any medium. In order to retrieve the reflection responses of both modes properly with full-3D interferometry in the frequency-wavenumber domain as well as in the frequency-space domain, additional data are required. Therefore, data of a crossline source was added.

Interferometry in the frequency-wavenumber domain produced more accurate results than interferometry in the frequency-space domain in both cases, i.e., synthesized-2D and full-3D interferometry. This may not be the case for real data because the assumption made for interferometry in the wavenumber domain (no horizontal variation of the medium) may be unrealistic. However, interferometry in the frequency-space domain requires a set of sources that illuminate the subsurface from as many angles as possible. Sources close to the edge or outside the receiver array are not properly sampled, i.e., the electromagnetic field for these sources is not recorded on all sides of the source for large enough offsets. This introduces artefacts, which finally disturb the retrieved reflection response. 
We computed the normalized amplitude of the electromagnetic fields and of the retrieved reflection responses in order to determine if the detectability of the reservoir is increased after interferometry has been applied. The normalized amplitude of the electromagnetic fields before interferometry are slightly above one, indicating that the reservoir is barely detectable. In contrast, the normalized amplitude of the TM-mode reflection response is at least two but features for a large offset range values larger than four. On the other hand, the TE-mode reflection response shows a similar normalized amplitude as the unprocessed electromagnetic fields. These two findings show, firstly, that interferometry is able to increase the detectability of the reservoir and, secondly, that the reservoir information is mainly in the TM-mode of the electromagnetic field.

For the decomposition, it is necessary to know the conductivity of the seabed. In our situation, the conductivity was required with a precision of $\pm 0.1 \mathrm{~S} / \mathrm{m}$. Overestimating the conductivity has a larger effect than underestimating it. The other crucial parameter in the interferometry scheme is the stabilization parameter. A stabilization parameter that is too large damps the amplitude of the retrieved reflection response. In the synthesized$2 \mathrm{D}$ cases as well as in the full-3D frequency-space-domain interferometry, a too small stabilization parameter introduces instabilities in the retrieved reflection response.

\subsection{Appendix A: The 2D decomposition algorithm}

The 2D decomposition algorithm requires only two components of the electromagnetic field. Equation 4.2 becomes

$$
\left(\begin{array}{c}
\tilde{P}^{T M+}\left(k_{x}, \omega\right) \\
\tilde{P}^{T M-}\left(k_{x}, \omega\right)
\end{array}\right)=\left(\begin{array}{cc}
L_{2} & L_{1} \\
L_{2} & -L_{1}
\end{array}\right)\left(\begin{array}{c}
\tilde{H}_{y}\left(k_{x}, \omega\right) \\
\tilde{E}_{x}\left(k_{x}, \omega\right)
\end{array}\right)
$$

where

$$
\begin{aligned}
L_{1} & =\sqrt{\frac{\eta_{1}}{2 \Gamma_{1}}} \text { and } \\
L_{2} & =\sqrt{\frac{\Gamma_{1}}{2 \eta_{1}}} .
\end{aligned}
$$

Thereby, $\Gamma_{1}=\sqrt{k_{x}^{2}+\eta_{1} \zeta_{1}}, \eta_{1}=\sigma_{1}+j \omega \epsilon_{1}$ and $\zeta_{1}=j \omega \mu_{1}$ with $\sigma_{1}, \epsilon_{1}$ and $\mu_{1}$ being the electric conductivity, electric permittivity and magnetic permeability, respectively. The subscript 1 indicates that the material parameters below the receivers are used. The angular frequency is given by $\omega=2 \pi f$ with $f$ being the natural frequency and $j=\sqrt{-1}$ is the imaginary unit. 


\section{Chapter 5}

\section{Time-lapse Controlled Source Electromagnetics using interferometry ${ }^{\mathrm{i}}$}

\section{Abstract}

In time-lapse Controlled Source Electromagnetics it is crucial that the source and the receivers are positioned at exactly the same location at all times of measurement. We use interferometry by multidimensional deconvolution (MDD) to overcome problems in repeatability of the source location. Interferometry by MDD redatums the source to a receiver location and replaces the medium above the receivers with a homogeneous halfspace. In this way, changes in the source position and changes of the conductivity in the waterlayer become irrelevant. The only remaining critical parameter to ensure a good repeatability of a Controlled Source Electromagnetic measurement is the receiver position.

\subsection{Introduction}

Subsurface exploration with seismic methods is able to delineate geologic structures, but it is not able to distinguish between a structure whose porous rock is water-filled and another structure that is filled with hydrocarbons. Since hydrocarbons have a much higher electric resistivity than water, they can be detected by electric methods. Therefore, it is useful to conduct a

${ }^{\mathrm{i}}$ This chapter has been compiled out of a journal paper in The Leading Edge, 30(5), p. 564-567 (Hunziker et al., 2011c) and a conference proceeding for the 2011 IEEE International Geoscience and Remote Sensing Symposium in Vancouver (Hunziker et al., 2011b). 
marine Controlled Source Electromagnetics (CSEM) survey, in addition to a seismic survey, in order to determine wether the structures located by seismics bear hydrocarbons or not. In frequency-domain marine CSEM an electric source is towed by a boat over a set of multicomponent receivers at the ocean bottom. The source emits continuously a monochromatic lowfrequency signal. A part of the resulting electric field is diffusing through the subsurface, samples possible resistors and is finally recorded at the receivers. For a more detailed introduction to CSEM, see Constable \& Srnka (2007).

In this chapter, we focus on time-lapse CSEM measurements for reservoir monitoring. Constable \& Weiss (2006) have shown that CSEM is sensitive to changes in thickness of a subsurface resistor, which hints at the feasibility of time-lapse CSEM. It was shown numerically, that changes in the reservoir response are measurable if the maximum repeatability error of the CSEM measurement is in the order of $1 \%$ to $2 \%$ (Orange et al., 2009; Wirianto et al., 2010). To achieve such a repeatability of the measurement, we propose to use interferometry by multidimensional deconvolution (MDD). Interferometry redatums arbitrarily distributed sources with random source strengths to a well-defined receiver array. In other words, it does not matter, where the sources are located in the ocean and therefore source-mispositioning issues become irrelevant. Furthermore, it replaces the overburden, i.e. everything above the receivers, with a homogeneous halfspace. Therefore, possible changes in seawater conductivity are removed as well and, generally, the detectability of changes in the subsurface is increased, because effects related to the air-water interface are removed. The only possibly problematic issue that remains in time-lapse CSEM is the repeatability of the receiver positions, but that should be easier to achieve than the repeatability of the source, because the receivers are stationary.

\subsection{Theory}

Interferometry by MDD can be divided into two parts: First, the recorded electromagnetic fields need to be decomposed into upward and downward decaying components, which was first done by Amundsen et al. (2006). We use an algorithm of Slob (2009), which in 3D needs all four horizontal components of the electromagnetic field. In this study, we use 2D transverse magnetic data, which means, that the source is infinitely long in the second spatial direction ( $y$-direction). In this case, the decomposition can be done with just two components, the inline electric component $E_{x}$ and the crossline magnetic component $H_{y}$. To perform the decomposition, the material parameters just below the ocean bottom, but not those of the ocean, are necessary. In 
the space-frequency domain, the upward decaying field $\hat{P}^{-}\left(x_{R}, x_{S}\right)$ is related with the downward decaying field $\hat{P}^{+}\left(x_{R}^{\prime}, x_{S}\right)$ through the reflection response $\hat{R}_{0}^{+}\left(x_{R}, x_{R}^{\prime}\right)$ :

$$
\hat{P}^{-}\left(x_{R}, x_{S}\right)=\int_{\partial D_{R}} \hat{R}_{0}^{+}\left(x_{R}, x_{R}^{\prime}\right) \hat{P}^{+}\left(x_{R}^{\prime}, x_{S}\right) d x_{R}^{\prime},
$$

where $x_{R}$ and $x_{R}^{\prime}$ are the coordinates of receiver stations, whereas $x_{S}$ is the source position. The circumflex denotes the space-frequency domain. The superscript ${ }^{+}$in the reflection response indicates that its origin is a downward decaying field and the subscript 0 represents the absence of heterogeneities above the receiver level. This equation can be rewritten in matrix notation as described by Berkhout (1982) as

$$
\hat{\mathbf{P}}^{-}=\hat{\mathbf{R}}_{0}^{+} \hat{\mathbf{P}}^{+} .
$$

Each column of the matrices contains various receiver positions for a fixed source position, while for the rows the situation is reversed.

In the second part, we solve equation 5.2 for the reflection response $\hat{\mathbf{R}}_{0}^{+}$, i.e. the scattered Green's function of the subsurface, by multidimensional deconvolution of the upward decaying field with the downward decaying field (Wapenaar et al., 2008b). This can for example be achieved with a leastsquares inversion:

$$
\hat{\mathbf{R}}_{\mathbf{0}}^{+}=\hat{\mathbf{P}}^{-}\left(\hat{\mathbf{P}}^{+}\right)^{\dagger}\left[\hat{\mathbf{P}}^{+}\left(\hat{\mathbf{P}}^{+}\right)^{\dagger}+\varepsilon^{2} \mathbf{I}\right]^{-1} .
$$

The superscript ${ }^{\dagger}$ denotes complex-conjugation and transposition and $\mathbf{I}$ is the identity matrix. The stabilization parameter $\varepsilon$ prevents the inversion from getting unstable.

In time-lapse CSEM, we apply interferometry by MDD to data recorded at all times of measurement in order to remove changes related to source position or salinity of the ocean. Then the retrieved subsurface reflection responses of two different times of measurement are compared. This is shown with three numerical examples in the next section.

\section{$5.3 \quad$ Results}

We model electromagnetic fields due to a unit source at two different times for a layered Earth model. The modelling setup at the first time of measurement $t_{1}$ is shown in Figure 5.1. The source is a unit source and towed $25 \mathrm{~m}$ above 
the receivers. The depths as well as the electric conductivities $\sigma$ of all layers are given in Figure 5.1. We model two different production methods. In both $20 \%$ of the reservoir has been produced at the second time of measurement $t_{2}$.

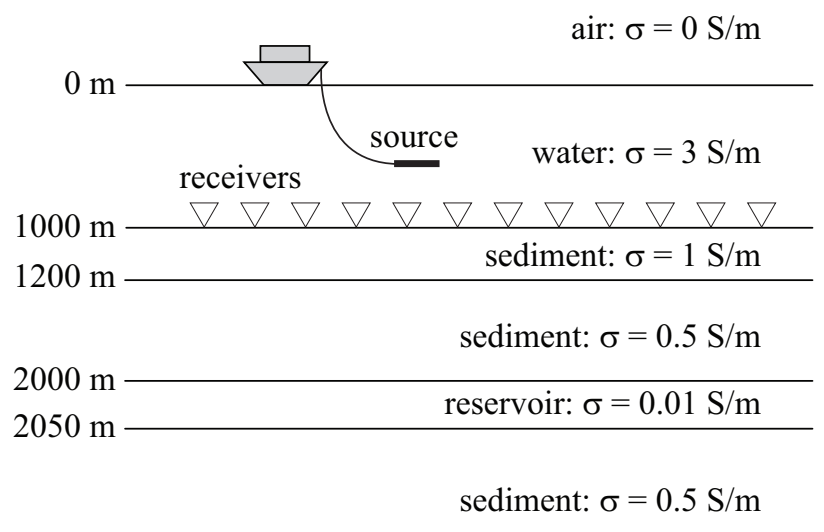

Figure 5.1: The modelling setup (not to scale). The source (black horizontal bar) is $25 \mathrm{~m}$ above the receivers (white triangles). The electric conductivity $\sigma$ is given in every layer.
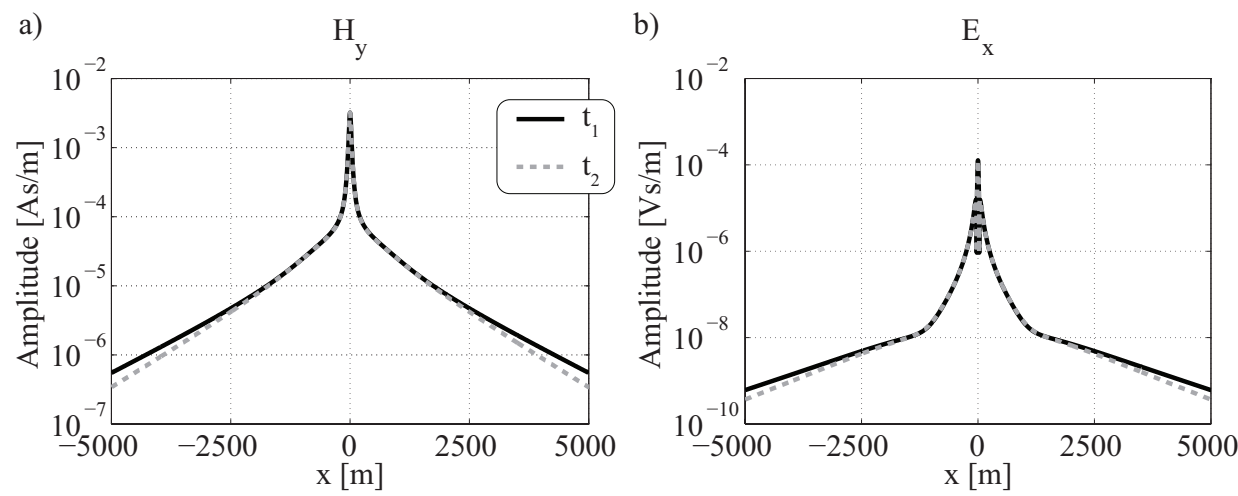

Figure 5.2: a) The crossline magnetic field $H_{y}$ and b) the inline electric field $E_{x}$ recorded at the first time of measurement $t_{1}$ (solid black curve) and at the second time of measurement $t_{2}$ (dashed grey curve) as a function of offset with respect to the source position for scenario 1. Note that the amplitude scale is different for the electric field as for the magnetic field. 


\subsubsection{Scenario 1}

In the first scenario, the oil content is reduced homogeneously throughout the complete reservoir. To compute the conductivity of the reservoir layer after partial production, we use Archie's law (Archie, 1942), which relates the electric conductivity of a porous rock $\sigma_{r}$ with its porosity $\phi$ and brine saturation $S$

$$
\sigma_{r}=\sigma_{b}(\phi S)^{2},
$$

where $\sigma_{b}$ is the conductivity of the brine $(6.25 \mathrm{~S} / \mathrm{m})$. Assuming a porosity of $28 \%$ and, after production, a brine saturation of $20 \%$, i.e. $20 \%$ of the oil has been produced, the electric conductivity of the reservoir layer is in the second time of measurement $t_{2} 0.02 \mathrm{~S} / \mathrm{m}$. A third dataset is recorded at the same time, but with the source towed $15 \mathrm{~m}$ higher than at $t_{1}$ and $t_{2}$. We refer

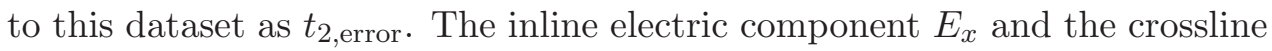
magnetic component $H_{y}$ are shown for the two times of measurement $t_{1}$ and $t_{2}$ in Figure 5.2.

The electromagnetic fields at the two times of measurement are very similar. A subtraction of the fields at $t_{2}$ from those at $t_{1}$ reveals the differences more clearly. Figure $5.3 \mathrm{a}$ and $5.3 \mathrm{~b}$ show the difference between $t_{1}$ and $t_{2}$ (solid black curve) and the difference between $t_{1}$ and $t_{2, \text { error }}$ (dashed grey curve), i.e. the difference between the measurement before production and the second measurement including the altered source position. The amplitude of the dashed grey curve is at small and intermediate offsets much larger than the one of the solid black curve. This means that the misplacement of the source has a larger impact on the recorded electromagnetic fields than the change of the conductivity of the reservoir layer from $0.01 \mathrm{~S} / \mathrm{m}$ to $0.02 \mathrm{~S} / \mathrm{m}$.

We apply interferometry by MDD to these three datasets in order to redatum the source. The differences of the retrieved reflection responses are depicted in Figure 5.3c. Since interferometry redatums the source to receiver positions, the effect of the mispositioning of the source is removed. Therefore, the solid black curve $\left(t_{1}-t_{2}\right)$ is identical with the dashed grey curve $\left(t_{1}-t_{2, \text { error }}\right)$. This is achieved without knowing the actual source location in both time-lapse measurements.

\subsubsection{Scenario 2}

In the second scenario also $20 \%$ of the oil is produced, but in this scenario the lowest part of the reservoir is swepted completely, whereas there is no change in oil content in the upper part. This bottom flooding scenario is modeled by 

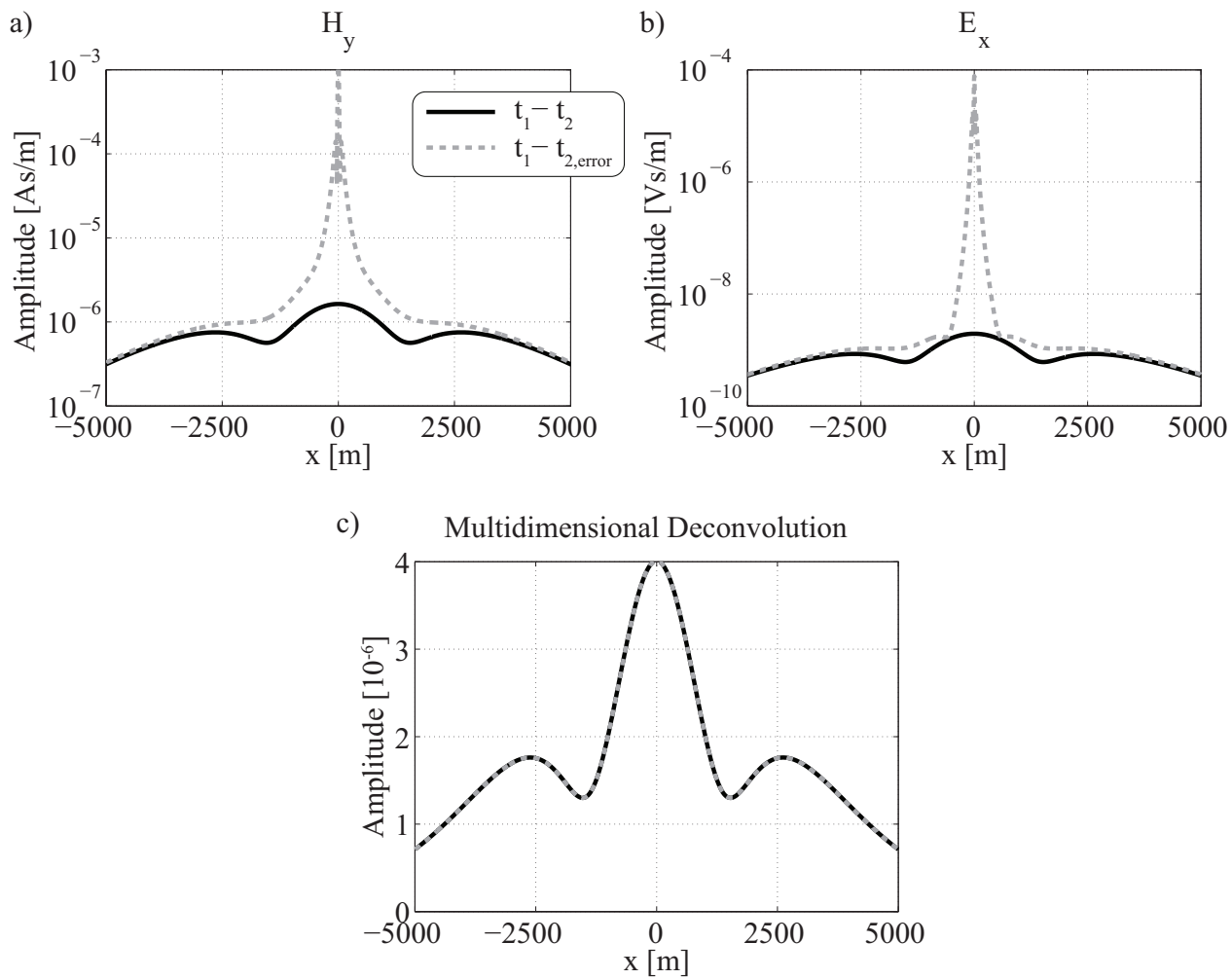

Figure 5.3: Difference between two times of measurement of scenario 1: a) magnetic field in crossline direction, b) electric field in inline direction and c) retrieved reflection response. Solid black curve: $t_{1}-t_{2}$; Dashed grey curve: $t_{1}-t_{2, \text { error. Note }}$ that the colours represent different quantities than in Figure 5.2.

using a $20 \%$ thinner reservoir without a change in the conductivity. Similar to the first scenario, we consider a time of measurement before production $t_{1}$ and a second time of measurement during production $t_{2}$. Additionally, we also model a dataset $t_{2 \text {,error }}$ with a different salinity of the ocean. This is simulated by changing the conductivity of the water layer from $3 \mathrm{~S} / \mathrm{m}$ to 3.2 $\mathrm{S} / \mathrm{m}$. The source is in all three datasets at the same position.

Figure 5.4a and 5.4b show the difference between the datasets during and before production. A comparison of the dashed grey curve $\left(t_{1}-t_{2, \text { error }}\right)$ with the solid black curve $\left(t_{1}-t_{2}\right)$ indicates, that the effect of the changed ocean salinity has a more significant impact on the data than the change in the reservoir thickness. This is especially at small and intermediate offsets the case. Also this effect can be removed by applying interferometry by MDD (Figure 5.4c). To achieve this, the conductivity of the ocean does not need to 

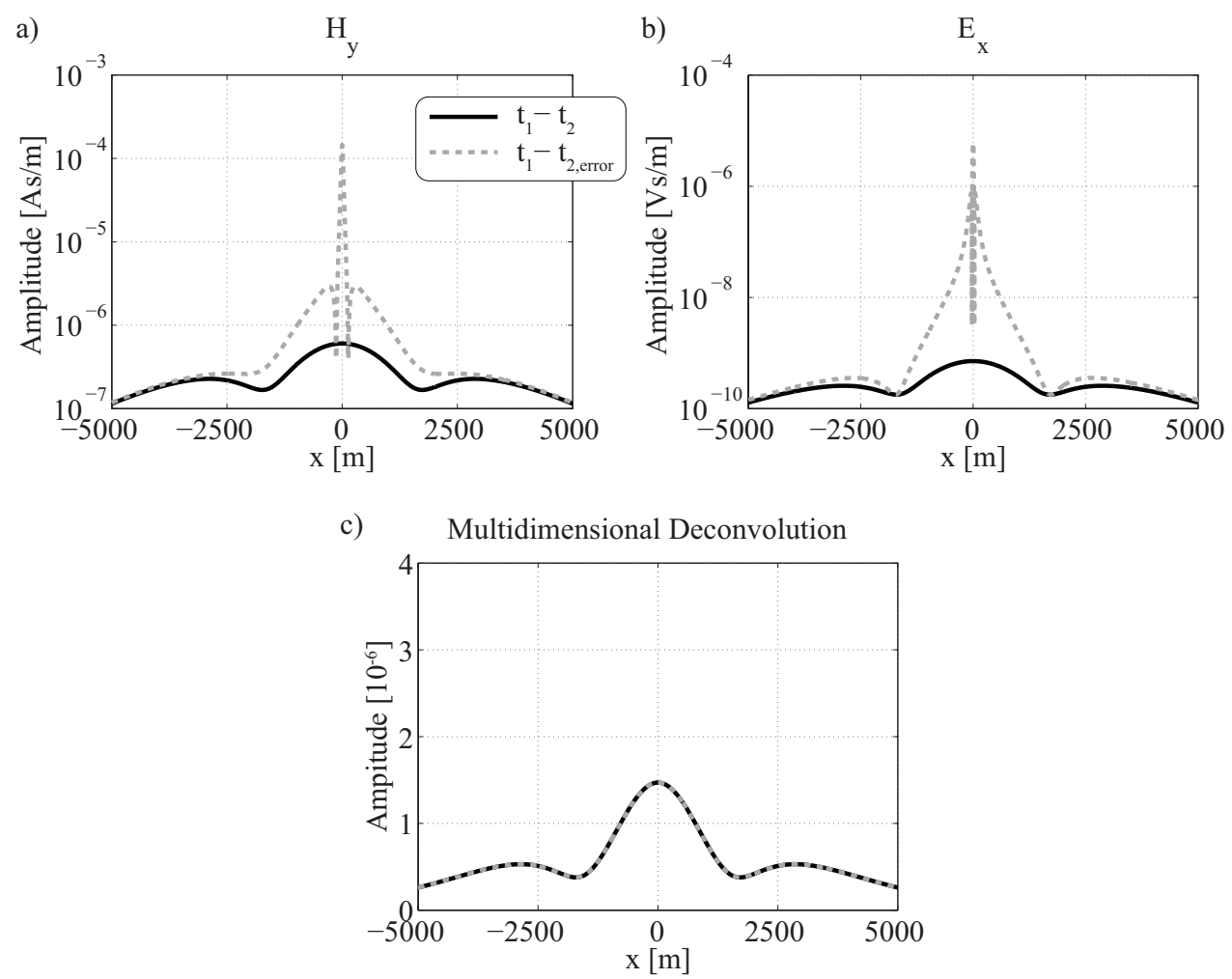

Figure 5.4: Same as Figure 5.3 for the second scenario.

be known. Only the conductivity just below the ocean bottom is required for the decomposition, which can be easily obtained from local measurements.

\subsubsection{Scenario 3}

The errors discussed in scenario 1 and 2 can also occur simultaneously. To demonstrate that these independent effects can also be removed with interferometry by MDD when appearing in combination, we created scenario 3 . As in the previous case, the oil is produced by bottom flooding. Additionally to the change in salinity of the ocean also the source is mispositioned. In other words, the dataset $t_{2 \text {,error }}$ combines the two errors from the previous two scenarios.

We apply interferometry by MDD to these dataset in order to remove both, the effects of the misplacement of the source and the increase in conductivity of the ocean. The differences of the measured fields as well as the difference of the retrieved reflection responses between the different times 

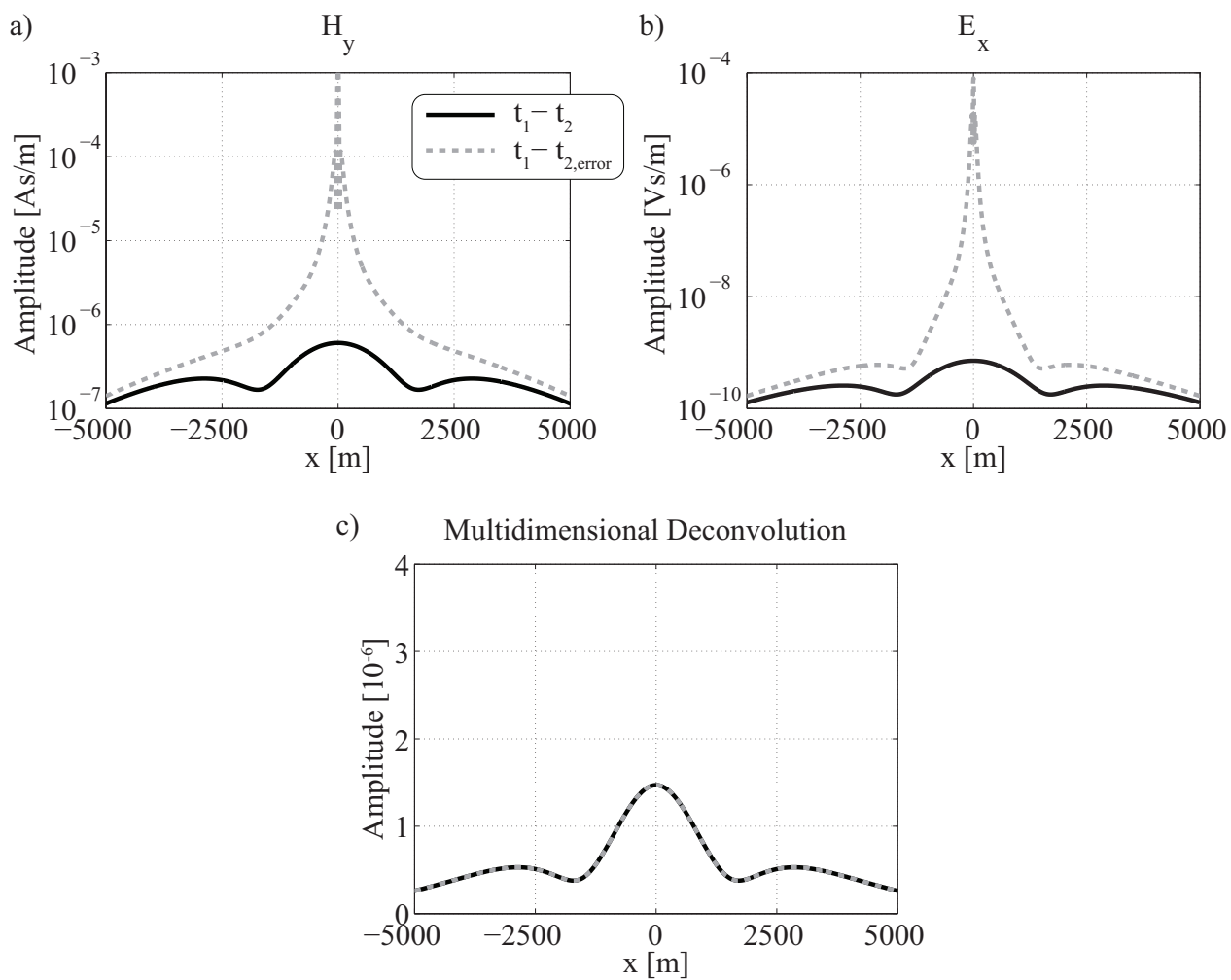

Figure 5.5: Same as Figure 5.3 for the third scenario.

of measurement are depicted in Figure 5.5. The difference of the retrieved reflection responses without any error (solid black curve, $t_{1}-t_{2}$ ) is identical with the difference of the retrieved reflection responses (dashed grey curve, $\left.t_{1}-t_{2 \text {,error }}\right)$. Consequently, the mispositioning of the source and the change in ocean salinity can be removed with interferometry in one go without knowing the actual source position nor the conductivity of the ocean.

\subsection{Conclusions}

Three time-lapse CSEM scenarios are modelled. Thereby two production methods, homogeneous sweep and bottom flooding, as well as two changes in the acquisition condition in the second time of measurement, mispositioning of the source and a change in ocean salinity leading to a change in the electric conductivity of the ocean, are simulated. These scenarios show that the changes in the reservoir during production can be detected with CSEM. 
However, the changes in the reservoir (conductivity or thickness) have a much smaller impact on the time-lapse data than the mispositioning of the source and the change of the ocean conductivity.

We apply interferometry by MDD to these datasets in order to retrieve the reflection response. By applying interferometry, the medium above the receivers is replaced by a homogeneous halfspace and the sources are redatumed to receiver positions. In other words, the changes in the acquisition condition are completely removed in the retrieved reflection response. Consequently, by applying interferometry by MDD to time-lapse CSEM data, the source positions do not need to be at the same locations, because they are redatumed to receiver positions. Furthermore, changes in the salinity of the ocean can also be removed successfully.

What remains as a critical acquisition parameter in time-lapse CSEM is the position of the receivers. Orange et al. (2009) concluded that receiver positions should be known and repeatable to less than $5-10 \mathrm{~m}$ accuracy. They propose to use permanent monuments at the ocean bottom to which the receivers can be attached by remotely operated vehicles. Andreis \& MacGregor (2010) simply assume the usage of a permanent receiver system to overcome this issue. 


\section{Chapter 6}

\section{Conclusions and outlook}

\subsection{Conclusions}

In this thesis, the benefits and limitations of interferometry by multidimensional deconvolution (MDD) for marine Controlled-Source Electromagnetics (CSEM) to suppress the airwave, the direct field and other events related to the air-water interface as well as to reduce source uncertainty are discussed. In the following, the main conclusions for each chapter are listed.

Chapter 2 introduces the reader to diffusive electromagnetic fields through visualisations of these fields in space and time. The appearance of the airwave as a function of antenna orientation is investigated. The following findings are crucial:

- Diffusive electromagnetic fields exhibit directionality in the sense of a temporal and spatial expanding high amplitude area, with the highest amplitude remaining at the source location. Consequently, techniques such as beam steering or decomposition in upward and downward decaying fields are not restricted to wavefields, but can also be applied to diffusive fields. Decomposition is relevant for this thesis because it is part of the interferometry workflow.

- Vertical antennas do not excite or record the airwave in a horizontally layered medium because the airwave has only horizontal components. However, already small deviations from verticality lead to a significant contribution of the airwave to the complete measured field.

Chapter 3 focuses on spatial sampling aspects and how these limitations can be overcome. Furthermore, noise and receiver positioning and orientation 
errors are discussed. The numerical examples are in 2D, assuming an infinitely long source in the crossline direction. It is assumed that the conclusions drawn are also valid for 3D data. The following are the major findings:

- The largest possible receiver spacing should be equal to or smaller than the larger of the following two parameters: (1) vertical distance between source and receivers and (2) the length of the source antenna.

- Moving the source vertically away from the receivers or using a longer source antenna limits the bandwidth of the data. This affects in the first place the direct field and reflections caused by interfaces close to the receivers, but it affects the reservoir response less because that already has a limited bandwidth due to the large depth of the reservoir.

- A large vertical source-receiver distance may not be practical, e.g., in a shallow sea. In practice, a long source antenna may hinder fast and efficient acquisition. In order to overcome the strict sampling rule and avoid large vertical source-receiver distances as well as long physical sources, the synthetic aperture source method is introduced. This method allows to create a synthetically long source antenna after the data have been acquired. No changes in the acquisition system are necessary, instead, standard CSEM data are used. The method exploits the oversampling on the source-side as it is common in CSEM.

- High levels of random noise hardly affect interferometry by MDD in combination with the synthetic aperture source, because the random noise stacks out during the creation of the synthetic aperture source.

- Receiver positioning and orientation errors limit the bandwidth of the retrieved reflection response. While positioning errors have only a small effect, orientation errors are severe. If all six components of the electromagnetic field as well as the orientation of the receiver antennas are measured, the receivers can be virtually rotated to the desired orientation, thus avoiding orientation errors.

Chapter 4 discusses synthesized-2D interferometry as well as full-3D interferometry in the frequency-wavenumber domain as well as in the frequencyspace domain using the synthetic aperture source. It also includes a section, which is devoted to effects of an inaccurate estimation of the conductivity of the ocean bottom for the decomposition or improperly chosen stabilization parameter for the least-squares inversion. The following conclusions are drawn: 
- Interferometry can only be applied to line data if the data are truly two-dimensional, i.e., due to an infinitely long source in the crossline direction. A sufficiently accurate infinitely long source in the crossline direction can be simulated by integrating over several source lines parallel to the receiver line assuming no or only little variation of the medium in crossline direction. In the example shown, 35 source lines proved to be sufficient. This is called synthesized-2D interferometry.

- In full-3D interferometry, reflection responses for the TM-mode, the TE-mode and the mode-conversions are retrieved. The wavenumberdomain implementation assumes a laterally invariant medium requiring one source in the centre of the receiver array, whereas the space-domain algorithm can be applied to any medium requiring a source distribution illuminating the medium from all sides. The disadvantage of the spacedomain algorithm is that it is very CPU intensive.

- In the numerical experiment, it has been shown that the conductivity of the ocean bottom needs to be known with a precision of $\pm 0.1 \mathrm{~S} / \mathrm{m}$. Underestimation of the conductivity is less severe than overestimation.

- A too large stabilization parameter in the least-squares inversion damps the amplitude of the retrieved reflection response. In the synthesized$2 \mathrm{D}$ cases as well as in full-3D frequency-space domain interferometry, a too small stabilization parameter introduces instabilities in the retrieved reflection response. To avoid the difficult choice of the stabilization parameter, inversion schemes based on singular value decomposition or iterated conjugate gradients could be used.

In chapter 5, interferometry by MDD for time-lapse CSEM data is discussed. Two production methods are simulated: homogeneous sweep and bottom flooding. The following conclusions can be drawn:

- For both methods, the changes in the reservoir due to production are measurable with CSEM. However, changes in the acquisition condition, in this case mispositioning of the source and a change in ocean salinity leading to a change of ocean conductivity, leave a stronger imprint in the data than changes in the reservoir due to production.

- Interferometry by MDD is able to remove these acquisition imprints without knowing the actual source position or the conductivity of the ocean. The only remaining critical parameter in time-lapse CSEM is the receiver location and the conductivity value just below the surface 
at the receiver locations. It was proposed in the literature to either attach the receivers to permanent monuments or install the receivers permanently on the ocean bottom to ensure receiver position repeatability.

\subsection{Outlook}

This thesis presents some advances in the field of interferometry by MDD for diffusive electromagnetic fields. Still, some work needs to be done before this becomes a standard tool for the oil and gas industry. Some of the possible future work is listed below:

- In this thesis, all examples consisted of a model with a laterally invariant earth made of various amounts of layers. Next, a more complicated earth structure, e.g., a box reservoir, should be modelled in order to apply interferometry by MDD to it. In that case, also the modeconversions are supposed to be non-zero. It will also be interesting to see how the reflection responses change as a function of lateral position.

- The retrieved reflection response should be inverted for the subsurface conductivity distribution and the result compared to an inversion based on standard CSEM data in order to answer the following questions: Is the retrieved conductivity distribution more accurate than based on standard CSEM data? Is the solution space, as assumed, better defined? Are less iterations necessary if an iterative scheme is used?

- Interferometry by MDD could also be applied to time-domain CSEM data. Technically, it is the same as applying interferometry to frequencydomain CSEM data, but just for several frequency slices.

- Design and implement an algorithm for the decomposition into upward and downward decaying fields in the space domain. This would allow to include lateral variations of the medium at the receiver level. 


\section{Bibliography}

Abramowitz, M., \& Stegun, I. A. 1972. Handbook of Mathematical Functions. 10th edition, Applied Mathematics Series 55, National Bureau of Standards, Washington, USA.

Amundsen, L., Løseth, L., Mittet, R., Ellingsrud, S., \& Ursin, B. 2006. Decomposition of electromagnetic fields into upgoing and downgoing components. Geophysics, 71, G211-G223.

Andréis, D., \& MacGregor, L. 2007. Time domain versus frequency domain CSEM in shallow water. 77th Annual International Meeting, SEG, Expanded Abstracts.

Andreis, D. L., \& MacGregor, L. M. 2010. Using CSEM to Monitor the Production of a Complex 3D Gas Reservoir - A Synthetic Case Study. 72nd EAGE Conference and Exhibition, Barcelona, Expanded Abstracts, C003.

Archie, G. E. 1942. The Electrical Resistivity Log as an Aid in Determining Some Reservoir Characteristics. Petroleum Transactions, AIME, 146, 5462 .

Avdeev, D. B. 2005. Three-dimensional electromagnetic modelling and inversion from theory to application. Surveys in Geophysics, 26, 767-799.

Bakulin, A., \& Calvert, R. 2006. The virtual source method: Theory and case study. Geophysics, 71, SI139-SI150.

Bannister, P. R. 1984. New simplified formulas for ELF subsurface-tosubsurface propagation. IEEE Journal of Oceanic Engineering, OE-9, 154-163.

Berkhout, A. J. 1982. Seismic Migration. Imaging of Acoustic Energy by Wave Field Extrapolation. Elsevier. 
Castagna, J. P., \& Swan, H. W. 1997. Principles of AVO crossplotting. The Leading Edge, 16(4), 337-344.

Chave, A. D., Constable, S. C., \& Edwards, R. N. 1991. Electrical exploration methods for the seafloor. In Nabighian, M. (Ed) Electromagnetic Methods in Applied Geophysics, Vol 2. Soc. Explor. Geophys., Tulsa.

Chen, J., \& Alumbaugh, D. L. 2011. Three methods for mitigating airwaves in shallow water marine controlled-source electromagnetic data. Geophysics, 76, F89-F99.

Constable, S. 2010. Ten years of marine CSEM for hydrocarbon exploration. Geophysics, 75, 75A67-75A81.

Constable, S., \& Srnka, L. J. 2007. An introduction to marine controlledsource electromagnetic methods for hydrocarbon exploration. Geophysics, 72(2), WA3-WA12.

Constable, S., \& Weiss, C. J. 2006. Mapping thin resistors and hydrocarbons with marine EM methods: Insights from 1D modeling. Geophysics, 71, G43-G51.

Dell'Aversana, P. 2007. Marine CSEM data interpretation: Pitfalls and possible solutions. The Leading Edge, 26(6), 686-691.

Draganov, D., Wapenaar, K., \& Thorbecke, J. 2006. Seismic interferometry: Reconstructing the earth's reflection response. Geophysics, 71, SI61-SI70.

Eidesmo, T., Ellingsrud, S., MacGregor, L. M., Constable, S., Sinha, M. C., Johansen, S., Kong, F. N., \& H., Westerdahl. 2002. Sea Bed Logging(SBL), a new method for remote and direct identification of hydrocarbon filled layers in deepwater areas. First Break, 20, 144-152.

Ellingsrud, S., Eidesmo, T., Johansen, S., C., Sinha M., MacGregor, L. M., \& Constable, S. 2002. The Meter Reader - Remote sensing of hydrocarbon layers by seabed logging (SBL): Results from a cruise offshore Angola. The Leading Edge, 21(10), 972-982.

Fan, Y., Snieder, R., Slob, E., Hunziker, J., Singer, J., Sheiman, J., \& Rosenquist, M. 2010. Synthetic aperture controlled source electromagnetics. Geophys. Res. Lett., 37, L13305.

Fan, Y., Snieder, R., Slob, E., Hunziker, J., \& Singer, J. 2011. Steering and focusing diffusive fields using synthetic aperture. Europhys. Lett., 95, 34006 . 
Frijlink, M., \& Wapenaar, K. 2010. Reciprocity Theorems for One-Way Wave Fields in Curvilinear Coordinate Systems. SIAM J. Imaging Sciences, 3, 390-415.

Grimbergen, J L T, Dessing, F J, \& Wapenaar, K. 1998. Modal expansion of one-way operators in laterally varying media. Geophysics, 63, 995-1005.

Harris, P., Du, Z., MacGregor, L., Olsen, W., Shu, R., \& Cooper, R. 2009. Joint interpretation of seismic and CSEM data using well log constraints: an example from the Luva Field. First Break, 27, 73-81.

Hesthammer, J., Stefatos, A., Boulaenko, M., Fanavoll, S., \& Danielsen, J. 2010. CSEM performance in light of well results. The Leading Edge, 29(1), $34-41$.

Holten, T., Flekkøy, E. G., Singer, B., Blixt, E. M., Hanssen, A., \& Måløy, K. J. 2009. Vertical source, vertical receiver, electromagnetic technique for offshore hydrocarbon exploration. First Break, 27, 89-93.

Holvik, E., \& Amundsen, L. 2005. Elimination of the overburden response from multicomponent source and receiver seismic data, with source designature and decomposition into PP-, PS-, SP-, and SS-wave responses. Geophysics, 70, S43-S59.

Hu, W., Abubakar, A., \& Habashy, T. M. 2009. Joint electromagnetic and seismic inversion using structural constraints. Geophysics, 74(6), R99R109.

Hunziker, J., Slob, E., \& Wapenaar, K. 2009. Controlled Source Electromagnetic Interferometry by multidimensional deconvolution: spatial sampling aspects in Sea Bed Logging. 71st EAGE Conference and Exhibition, Expanded Abstracts.

Hunziker, J., Slob, E., \& Mulder, W. 2011a. Effects of the airwave in timedomain marine controlled-source electromagnetics. Geophysics, 76(4), F251-F261.

Hunziker, J., Slob, E., \& Wapenaar, K. 2011b. Interferometry by multidimensional deconvolution for time-lapse controlled source electromagnetics. 2011 IEEE International Geoscience and Remote Sensing Symposium, Vancouver.

Hunziker, J., Slob, E., Wapenaar, K., Fan, Y., \& Snieder, R. 2011c. Timelapse controlled-source electromagnetics using interferometry. The Leading Edge, 30(5), 564-567. 
Hunziker, J., Slob, E., Fan, Y., Snieder, R., \& Wapenaar, K. 2012. Twodimensional Controlled-Source Electromagnetic Interferometry by multidimensional deconvolution: spatial sampling aspects. Geophysical Prospecting, (accepted).

Johansen, S., Brauti, K., Fanavoll, S., Amundsen, H., A., Wicklund T., Danielsen, J., Gabrielsen, P. T., Lorentz, L., Frenkel, M., Dubois, B., Christensen, O., Elshaug, K., \& A., Karlsen S. 2008. How EM survey analysis validates current technology, processing and interpretation methodology. First Break, 26, 83-88.

Jones, D. S. 1994. Methods in electromagnetic wave propagation. Clarendon.

Key, K. 2009. 1D inversion of multicomponent, multifrequency marine CSEM data: Methodology and synthetic studies for resolving thin resistive layers. Geophysics, 74(2), F9-F20.

King, R. W., Owens, M., \& Wu, T. 1992. Lateral electromagnetic waves, Theory and applications to communications, geophysical exploration, and remote Sensing. Springer-Verlag New York, Inc.

Kinneging, N. A., Budejicky, V., Wapenaar, C. P. A., \& Berkhout, A. J. 1989. Efficient 2D and 3D shot record redatuming. Geophysical Prospecting, 37, 493-530.

Kong, J. A. 1986. Electromagnetic Wave Theory. John Wiley \& Sons.

Løseth, L. O., Amundsen, L., \& Jenssen, A. J. K. 2010. A solution to the airwave-removal problem in shallow-water marine EM. Geophysics, 75, A37-A42.

Maaø, F. A., \& Nguyen, A. K. 2010. Enhanced subsurface response for marine CSEM surveying. Geophysics, 75, A7-A10.

MacGregor, L., \& Sinha, M. 2000. Use of marine controlled-source electromagnetic sounding for sub-basalt exploration. Geophysical Prospecting, 48, 1091-1106.

MacGregor, L., Andreis, D., Tomlinson, J., \& Barker, N. 2006. Controlledsource electromagnetic imaging on the Nuggets-1 reservoir. The Leading Edge, 25(8), 984-992.

Mittet, R. 2008. Normalized amplitude ratios for frequency-domain CSEM in very shallow water. First Break, 26, 47-54. 
Mulder, W. A. 2006. A multigrid solver for 3D electromagnetic diffusion. Geophysical Prospecting, 54, 633-649.

Mulder, W. A., Wirianto, M., \& Slob, E. C. 2008. Time-domain modeling of electromagnetic diffusion with a frequency-domain code. Geophysics, 73, F1-F8.

Nabighian, M. N. 1988. Electromagnetic Methods in Applied Geophysics. Society of Exploration Geophysicists, Tulsa.

Nordskag, J. I., \& Amundsen, L. 2007. Asymptotic airwave modeling for marine controlled-source electromagnetic surveying. Geophysics, 72, F249F255.

Nordskag, J. I., Amundsen, L., Løseth, L., \& Holvik, E. 2009. Elimination of the water-layer response from multi-component source and receiver marine electromagnetic data. Geophysical Prospecting, 57, 897-918.

Orange, A., Key, K., \& Constable, S. 2009. The feasibility of reservoir monitoring using time-lapse marine CSEM. Geophysics, 74, F21-F29.

Plessix, R. É., \& Mulder, W. A. 2008. Resistivity imaging with controlledsource electromagnetic data: depth and data weighting. Inverse Problems, 24, 1-22.

Raiche, A. T., \& Coggon, J. H. 1975. Analytic Green's Tensors for Integral Equation Modelling. Geophys. J. R. astr. Soc., 42, 1035-1038.

Schuster, G. 2009. Seismic Interferometry. Cambridge University Press.

Schuster, G. T., Yu, J., Sheng, J., \& Rickett, J. $2004 . \quad$ Interferometric/daylight seismic imaging. Geophysical Journal International, 157, 838-852.

Sickle, K. van, \& Valusek, J. E. 1990. AVO analysis of 3-D seismic data identifies untested reservoirs in old gas field. The Leading Edge, 9(7), 1822 .

Slob, E. 2009. Interferometry by Deconvolution of Multicomponent Multioffset GPR Data. IEEE Transactions on Geoscience and Remote Sensing, 47, 828-838.

Slob, E., Draganov, D., \& Wapenaar, K. 2007a. Interferometric electromagnetic Green's functions representations using propagation invariants. Geophysical Journal International, 169, 60-80. 
Slob, E., Snieder, R., \& Wapenaar, K. 2007b. Interferometry in dissipative media: Addressing the shallow sea problem for Seabed Logging applications. 7rth Annual International Meeting, SEG, Expanded Abstracts.

Slob, E., Hunziker, J., \& Mulder, W. 2010. Green's tensors for the diffusive electric field in a VTI half-space. Progress in Electromagnetics Research, 107, 1-20.

Snieder, R. 2006. Extracting the Green's function of attenuating heterogeneous acoustic media from uncorrelated waves. Journal of the Acoustical Society of America, 121, 2637-2643.

Um, E. S., \& Alumbaugh, D. L. 2007. On the physics of the marine controlledsource electromagnetic method. Geophysics, 72, WA13-WA26.

Ursenbach, C. P., \& Stewart, R. R. 2008. Two-term AVO inversion: Equivalences and new methods. Geophysics, 73(6), C31-C38.

van den Berg, P. M., Abubakar, A., \& Habashy, T. M. 2008. Removal of sea-surface-related wavefields and source replacement in CSEM data processing. 78th Annual International Meeting, SEG, Expanded Abstracts.

van der Neut, J., Hunziker, J., Wapenaar, K., \& Slob, E. 2008. Controlledsource seismic interferometry by multi-dimensional deconvolution: stability aspects with various numbers of sources and receivers. 78th Annual International Meeting, SEG, Expanded Abstracts.

Wait, J. R. 1959. Propagation of electromagnetic pulses in a homogeneous conducting earth. Appl. Sci. Res., 8, 213-253.

Wapenaar, C. P. A., \& Grimbergen, J. L. T. 1996. Reciprocity theorems for one-way wavefields. Geophysical Journal International, 127, 169-177.

Wapenaar, K. 2004. Retrieving the elastodynamic Green's function of an arbitrary inhomogeneous medium by cross correlation. Physical Review Letters, 93, 254301-1 - 254301-4.

Wapenaar, K., van der Neut, J., \& Ruigrok, E. 2008a. Passive seismic interferometry by multidimensional deconvolution. Geophysics, 73, A51-A56.

Wapenaar, K., Slob, E., \& Snieder, R. 2008b. Seismic and electromagnetic controlled-source interferometry in dissipative media. Geophysical Prospecting, 56, 419-434. 
Wapenaar, K., Draganov, D., Snieder, R., Campman, X., \& Verdel, A. 2010a. Tutorial on seismic interferometry: Part 1 - Basic principles and applications. Geophysics, 75, 75A195-75A209.

Wapenaar, K., Slob, E., Snieder, R., \& Curtis, A. 2010b. Tutorial on seismic interferometry: Part 2 - Underlying theory and new advances. Geophysics, 75, 75A211-75A227.

Weiss, C. J. 2007. The fallacy of the "shallow-water problem" in marine CSEM exploration. Geophysics, 72, A93-A97.

Wilt, M., \& Alumbaugh, D. 1998. Electromagnetic methods for development and production: State of the art. The Leading Edge, 17(4), 487-487.

Wirianto, M., Mulder, W. A., \& Slob, E. C. 2010. A feasibility study of land CSEM reservoir monitoring in a complex 3-D model. Geophysical Journal International, 181, 741-755.

Wirianto, M., Mulder, W. A., \& Slob, E. C. 2011. Exploiting the airwave for time-lapse reservoir monitoring with CSEM on land. Geophysics, 76(3), A15-A19.

Wright, D., Ziolkowski, A., \& Hobbs, B. 2002. Hydrocarbon detection and monitoring with a multicomponent transient electromagnetic (MTEM) survey. The Leading Edge, 21(9), 852-864.

Yilmaz, Ö. 1987. Seismic Data Processing. Society of Exploration Geophysicists, Tulsa, USA.

Zhdanov, M., \& Hursan, G. 2000. 3D electromagnetic inversion based on quasi-analytical approximation. Inverse Problems, 16, 1297-1322.

Ziolkowski, A. 2007. Developments in the transient electromagnetic method. First Break, 25, 99-106.

Ziolkowski, A., \& Wright, D. 2007. Removal of the air wave in shallow marine transient EM Data. 77th Annual International Meeting, SEG, Expanded Abstracts. 


\section{Summary}

\section{Marine Controlled-Source Electromagnetic Interferometry}

Controlled Source Electromagnetics (CSEM) is a geophysical exploration method that is used as a derisking tool by the oil- and gas-industry. Thereby, the sensitivity of the electromagnetic fields to resistors in the subsurface, which are possible hydrocarbon reservoirs, is exploited. In the marine case, the method consists of an electric source, which is towed by a boat over a set of multicomponent receiver stations located at the ocean bottom. The signal diffuses from the source not only through the subsurface to the receiver stations, but also directly through the water layer and indirectly via the airwater interface. Signals on these travelpaths can conceal a response from a possible hydrocarbon reservoir.

Interferometry by multidimensional deconvolution (MDD) removes in the ideal case the direct field, replaces the medium above the receivers with a homogeneous halfspace and redatums the source to the receiver locations. In other words, the scattered Green's function of the subsurface, also known as the reflection response, is retrieved. In this thesis, the benefits and limitations of interferometry by MDD as an intermediate step in the CSEM processing flow are investigated. The goal of this intermediate step is to suppress the direct field and any signal interacting with the air-water interface as well as to reduce the source uncertainty.

Interferometry by MDD roughly consists of two steps: Firstly, the multicomponent electromagnetic fields are decomposed into upward and downward decaying fields at the receiver level. Secondly, the reflection response is retrieved by deconvolving the upward decaying field with the downward decaying field. In order to perform the decomposition, the electromagnetic fields need to be sampled properly. That means dense enough for a sufficiently large offset range and without gaps. Numerical experiments have shown, that the largest possible receiver sampling distance has to be smaller than or equal to the larger of the two parameters: (1) The vertical distance between the 
source and the receivers and (2) the source antenna length.

These sampling criteria are rather strict and difficult to apply in practice. Increasing the vertical distance between the source and the receivers may not be possible in various situations, e.g., in a shallow marine case. Handling a physically long source antenna may also be difficult in practice and hinder fast and efficient data acquisition. The problem can be solved by using the synthetic aperture source concept, which overcomes undersampling on the receiver side by exploiting oversampling on the source side. This allows to create synthetically long sources after the data have been acquired using standard acquisition geometries.

The decomposition also requires grid data. Data recorded with a line of receivers can only be decomposed into upward and downward decaying fields if the source is infinitely long in the crossline direction. Such a source can be simulated by integrating over a set of source lines parallel to, but off the receiver line. Interferometry based on such data is called synthesized-2D interferometry, because the $2 \mathrm{D}$ data used are created synthetically from $3 \mathrm{D}$ data.

Interferometry by MDD can be implemented in the wavenumber or space domains. The wavenumber-domain implementation assumes the medium to be laterally invariant. Therefore, one source position is enough to solve for the reflection response and the deconvolution is reduced to an elementwise division. In contrast, the space-domain implementation is applicable to any medium without restrictions, but it requires that the medium is illuminated by many sources distributed over the target area. Furthermore, a matrix inversion needs to be performed in order to retrieve the reflection response.

In practice, interferometry can be used to suppress the airwave and any interactions of the signal with the air-water interface in order to get a clearer signal from the reservoir in the exploration case. If the data are supposed to be inverted for the subsurface conductivity distribution, interferometry is very helpful because it can reduce the source uncertainty by redatuming the source positions to the receiver locations. In reservoir monitoring using repeated surveys, it is crucial to know the source location very precise in order to have the source at the same locations in all measurements. This is normally not the case in CSEM. When interferometry is applied, it does not matter anymore where the source is located in subsequent surveys because it is redatumed to a receiver location. Clearly, this approach assumes that the receivers are at the same location in both surveys. Since interferometry replaces the medium above the receivers by a homogeneous halfspace with only knowing the material parameters at the receiver level, effects caused by changes in ocean salinity between several surveys can be suppressed as well. 


\section{Samenvatting}

\section{Marine elektromagnetische interferometrie met gecontroleerde bron- nen}

Elektromagnetisme met gecontroleerde bronnen is een geofysische exploratiemethode die door de olie- en gasindustrie wordt gebruikt om risico's te verkleinen. Daarbij wordt gebruikgemaakt van de gevoeligheid van elektromagnetische velden voor weerstanden in de ondergrond zoals mogelijke koolwaterstofreservoirs. In de marine situatie bestaat de methode uit een elektrische bron die door een schip over een serie van meercomponenten ontvangers getrokken wordt. De ontvangers zijn op de bodem van de oceaan geplaatst. Het signaal diffundeert vanaf de bron niet alleen door de ondergrond naar de ontvangers, maar ook direct door het water en indirect langs het lucht-water grensvlak. Signalen van deze verspreidingspaden kunnen het signaal, dat op een mogelijk koolwaterstofreservoir weerkaatst is, overschaduwen.

Interferometrie door multidimensionale deconvolutie verwijdert in de ideale situatie het directe veld, vervangt het medium boven de ontvangers door een homogene halfruimte en verplaatst de bron naar een ontvangerpositie. Met andere woorden, de verstrooide Greense functie van de ondergrond, ook bekend als reflectieresponsie, wordt gereconstrueerd. In dit proefschrift worden de voordelen en beperkingen van interferometrie door multidimensionale deconvolutie als een tussenstap in het verwerken van elektromagnetische data onderzocht. Deze tussenstap heeft als doel het directe veld en iedere interactie van het signaal met het lucht-water grensvlak te onderdrukken en onzekerheden in de bronpositie te verminderen.

Interferometrie door multidimensionale deconvolutie bestaat grofweg uit twee stappen: Eerst wordt het meercomponenten elektrische veld op het ontvangerniveau in omhoog en omlaag uitdempende delen gesplitst. Ten tweede wordt de reflectieresponsie door deconvolutie van het omhoog uitdempende veld met het omlaag uitdempende veld gereconstrueerd. Om de splitsing 
uit te voeren, moet het elektromagnetische veld goed bemonsterd zijn. Dat betekent dicht genoeg over een voldoend grote afstand en zonder hiaten. Numerieke experimenten hebben laten zien, dat de grootst mogelijke afstand tussen de ontvangers kleiner dan of gelijk aan de grootste van de volgende twee parameters moet zijn: (1) De verticale afstand tussen de bron en de ontvangers en (2) de lengte van de bronantenne.

Deze bemonstercriteria zijn nogal streng en in de praktijk moeilijk toe te passen. Het vergroten van de verticale afstand tussen de bron en de ontvangers is in veel situaties niet mogelijk, zoals bijvoorbeeld in een ondiepe zee. Het gebruiken van een fysiek lange bron kan in de praktijk ook moeilijk zijn en een snelle data-acquisitie belemmeren. Het probleem kan opgelost worden door het gebruik van het concept van bronnen met geconstrueerde belichtingshoek, die het tekort van monsters aan de ontvangerkant door het gebruik van een overschot aan monsters aan de bronkant compenseert. Dit laat het creëren van kunstmatig lange bronnen toe nadat de data met standaard opnamegeometrieën opgenomen zijn.

De splitsing in omhoog en omlaag uitdempende delen vereist dat de data op een raster opgenomen zijn. Data, die met een lijn van ontvangers zijn opgenomen, kunnen alleen in omhoog en omlaag uitdempende delen gesplitst worden als de bron oneindig lang is in de richting loodrecht op de ontvangerlijn. Een dergelijke bron kan gesimuleerd worden door te integreren over meerdere bronlijnen, die op verschillende afstanden parallel aan de ontvangerlijn liggen. Interferometrie, die op zulke data is gebaseerd, wordt synthetische $2 \mathrm{D}$ interferometrie genoemd, omdat de gebruikte $2 \mathrm{D}$ data synthetisch uit $3 \mathrm{D}$ data gecreëerd zijn.

Interferometrie door multidimensionale deconvolutie kan zowel in het golfgetal- als ook in het ruimtedomein geïmplementeerd worden. De golfgetaldomeinimplementatie neemt aan, dat het medium in horizontale richting niet verandert. Daarom is één bronpositie voldoende om de reflectieresponsie te reconstrueren en vereenvoudigt de deconvolutie tot een elementsgewijze deling. De ruimtedomeinimplementatie kan echter zonder beperkingen op ieder medium toegepast worden, maar vereist dat het medium door veel bronnen, verdeeld over het objectgebied, belicht wordt. Bovendien moet een matrixinversie uitgevoerd worden om de reflectieresponsie te reconstrueren.

In de praktijk kan interferometrie gebruikt worden om de luchtgolf en andere interacties van het signaal met het lucht-water grensvlak te onderdrukken om voor de exploratie een duidelijker signaal van het reservoir te verkrijgen. Als de data geïnverteerd worden voor de verdeling van de ondergrondse geleidbaarheid, kan interferometrie heel nuttig zijn omdat het de onzekerheid van de bronpositie vermindert door de bronnen naar ontvan- 
gerposities te verplaatsen. Bij het monitoren van de reservoirproductie met herhaalde metingen is het van groot belang de precieze bronpositie te kennen om de bron in alle metingen op dezelfde locatie te kunnen plaatsen. Dit is doorgaans niet het geval in elektromagnetisme met gecontroleerde bronnen. Als interferometrie op de data toegepast wordt, is het niet meer belangrijk waar de bron zich in de achtereenvolgende metingen bevindt, omdat de bron naar een ontvangerpositie verplaatst wordt. Het is duidelijk dat deze methode veronderstelt dat de ontvangers zich in alle metingen op dezelfde plaats bevinden. Omdat interferometrie het medium boven de ontvangers door een homogene halfruimte vervangt, waarbij alleen de materiaalparameters op het niveau van de ontvangers bekend moeten zijn, kunnen ook effecten, veroorzaakt door veranderingen in het zoutgehalte van de oceaan, onderdrukt worden. 


\section{Acknowledgements}

First of all, I would like to thank my main supervisor and promotor Evert Slob for always having had time for my questions. His optimistic view on the project has always encouraged and inspired me. I would also like to thank Kees Wapenaar, my second promotor, for his support during the last four years and for critically checking most of my publications. I owe many thanks to Roel Snieder who made it twice possible for me to visit the Center for Wave Phenomena at Colorado School of Mines in Golden, Colorado. I also thank him and Yuanzhong Fan for the very productive collaboration. Many thanks also go to Wim Mulder. I enjoyed very much working with him on the paper which is reprinted in this thesis as chapter 2. I would also like to thank him and Marwan Wirianto for allowing me to experiment with their numerical electromagnetic modelling code. Many thanks also to Jan Thorbecke for his help with coding emmod and to Guus Lohlefink for managing the various clusters I was working on. I would also like to acknowledge the members of my $\mathrm{PhD}$ committee for taking the time to read my thesis and providing interesting comments. Thanks also to Lydia, Margot, Marlijn, Ralf and all other staff members for their help and support.

Thanks to all my colleagues at the department and the other DOGS members for the very enjoyable four years: Adriaan, Aletta, Alex, Ali, Alimzhan, Amerjeet, Andrea (2+s), Araz, Asiya, Bobby, Carlos, Christiaan, Chunfang, Claudio, Daria, Dennis, Dessislava, Deyan, Duddy, Dylan, Elham, Elmer, Geertje, Guus, Huajun, Ilja, Jan, Joost, Karel, Maria, Mario, Martijn, Marwan, Mattia, Menne, Mohsen, Niels, Nihed, Panos, Pantelis, Patrick, Petar, Ralph, Rik, Silvian, Thomas, Tomohide, Tristan, Wiebke, Wieske and Xiaoxi. I enjoyed very much the apple breaks, DOGS drinks and trips, conferences, skiing in Zoetermeer and other activities. Especially I would like to thank Dennis for the many thrilling Atari battles and for letting me join to excursions to all kinds of places in the Netherlands between the lowest point (somewhere in the Zuidplaspolder, $-6.76 \mathrm{~m}$ ) and the highest point (Vaalserberg, $322.7 \mathrm{~m}$ ). Special thanks also to Deyan with whom I enjoyed many 
classical science-fiction movies as well as uncountable discussions about art, architecture, music, philosophy, science and washing machine maintenance. Many thanks also to Elmer for a lot of interesting discussions, for being the first person who had the patience to listen to my Dutch for a complete conversation and for correcting the Dutch translation of the propositions. Joost has been a very inspiring colleague on the project. I will also never forget all the trips we did together and the good collaboration we had in the last four years. I would also like to thank Karel, my long-term office neighbour, for the very insightful collaboration and for correcting the Dutch translation of the summary of this thesis. Unforgettable is also Nihed who made the Netherlands a truly welcoming place to me and never gave up trying to teach me some cooking skills.

A very nice part of my $\mathrm{PhD}$ was the time that I have spent in Colorado. I thank all my colleagues at Colorado School of Mines for letting me be part of their group. I owe much thanks to Valerie Walker at whose place I lived during my time in Golden. She did a lot to make my stay at the feet of the Rocky Mountains enjoyable. I would also like to thank the Bode family for the various skiing trips and other events I was invited to.

I would like to thank my family back in Switzerland for their 30 year long support. Without them all my projects would not have been possible. Finally, I would like to thank my darling Johanna for being my muse when I need inspiration and my sunshine when I am standing in the rain. 


\section{Curriculum Vitae}

\section{Personal information}

Name Jürg Werner Hunziker

Date of birth 15th of November 1982

Place of birth Lucerne, Switzerland

\section{Education and work}

2008-2012 PhD in Applied Geophysics and Petrophysics

Delft University of Technology, Delft, The Netherlands

2007 Ground Penetrating Radar internship

University of Miami, USA

2002-2007 MSc in Earth-Sciences

ETH Zürich, Switzerland

1995-2002 Matura

Gymnasium Alpenquai, Lucerne, Switzerland

\section{Journal papers (first author)}

- Hunziker, J., Slob, E., Fan, Y., Snieder, R., and Wapenaar, K., 2012, Electromagnetic Interferometry in three dimensions in wavenumber and space domains: Geophysics, (submitted).

- Hunziker, J., Slob, E., Fan, Y., Snieder, R., and Wapenaar, K., 2012, Two-dimensional controlled-source electromagnetic interferometry by multidimensional deconvolution: spatial sampling aspects: Geophysical Prospecting, (accepted).

- Hunziker, J., Slob, E., Wapenaar, K., Fan, Y., and Snieder, R., 2011, Time-lapse controlled-source electromagnetics using interferometry: The 
Leading Edge, Vol. 30, (5), 564-567.

- Hunziker, J., Slob, E., and Mulder, W., 2011, Effects of the airwave in time-domain marine controlled-source electromagnetics: Geophysics, Vol. 76, (4), F251-F261.

\section{Journal papers (co-author)}

- Fan, Y., Snieder, R., Slob, E., Hunziker, J., Singer, J., Sheiman, J., and Rosenquist, M., 2012, Increasing the sensitivity of controlled source electromagnetics with synthetic aperture: Geophysics, Vol. 77, (2), E135-E145.

- Wapenaar, K., van der Neut, J., Ruigrok, E., Draganov, D., Hunziker, J., Slob, E., Thorbecke, J., and Snieder, R., 2011, Seismic interferometry by crosscorrelation and by multi-dimensional deconvolution: a systematic comparison: Geophysical Journal International, Vol. 185, $1335-1364$.

- Fan, Y., Snieder, R., Slob, E., Hunziker, J., and Singer, J., 2011, Steering and focusing diffusive fields using synthetic aperture: EPL, Vol. 95,34006 .

- Minato, S., Matsuoka, T., Tsuji, T., Draganov, D., Hunziker, J., and Wapenaar, K., 2011, Seismic interferometry using multidimensional deconvolution and crosscorrelation for crosswell seismic reflection data without borehole sources: Geophysics, Vol. 76, (1), SA19-SA34.

- Fan, Y., Snieder, R., Slob, E., Hunziker, J., Singer, J., Sheiman, J., and Rosenquist, M., 2010, Synthetic aperture controlled source electromagnetics: Geophysical Research Letters, Vol. 37, L13305.

- Slob, E., Hunziker, J., and Mulder, W. A., 2010, Green's tensors for the diffusive electric field in a VTI half-space: Progress In Electromagnetics Research, Vol. 107, 1-20.

\section{Conference abstracts (first author)}

- Hunziker, J., Slob, E., Fan, Y., Snieder, R., and Wapenaar, K., 2012, Synthesized 2D CSEM-interferometry using automatic source line determination, 74th annual EAGE meeting, Copenhagen, P-268. 
- Hunziker, J., Slob, E., Wapenaar, K., Fan, Y., and Snieder, R., 2011, Effects of noise on CSEM interferometry with synthetic aperture sources, 81st annual SEG meeting, San Antonio, 614-618.

- Hunziker, J., Fan, Y., Slob, E., Wapenaar, K., and Snieder, R., 2011, CSEM interferometry using a synthetic aperture source, 73rd annual EAGE meeting, Vienna, P-162.

- Hunziker, J., Slob, E., and Wapenaar, K., 2011, Interferometry by multidimensional deconvolution for time-lapse controlled source electromagnetics, Proceedings of the 2011 IEEE International Geoscience and Remote Sensing Symposium, Vancouver, 1451.

- Hunziker, J., and Slob, E., 2010, 4D visualization of the airwave in a homogeneous halfspace, 80th annual SEG meeting, Denver, 905-909.

- Hunziker, J., van der Neut, J., Slob, E., Wapenaar, K., Fan, Y., and Snieder, R., 2010, Controlled source electromagnetic interferometry: the illumination function, 80th annual SEG meeting, Denver, 853-858.

- Hunziker, J., Fan, Y., Slob, E., Wapenaar, K., and Snieder, R., 2010, Solving spatial sampling problems in 2D-CSEM interferometry using elongated sources, 72nd annual EAGE meeting, Barcelona, P-083.

- Hunziker, J., van der Neut, J., Slob, E., and Wapenaar, K., 2009, Controlled source interferometry with noisy data, 79th annual SEG meeting, Houston, 689-693.

- Hunziker, J., Slob, E.C., and Wapenaar, K., 2009, Controlled source electromagnetic interferometry by multidimensional deconvolution - Spatial sampling aspects, 71st annual EAGE meeting, Amsterdam, P-074.

\section{Conference abstracts (co-author)}

- Wapenaar, K., Ruigrok, E., van der Neut, J., Draganov, D., Hunziker, J., Slob, E., Thorbecke, J., and Snieder, R., 2010, Green's function representation for seismic interferometry by deconvolution, 80th annual SEG meeting, Denver, 3972-3978.

- Fan, Y., Snieder, R., Slob, E., Hunziker, J., Singer, J., Sheiman, J., and Rosenquist, M., 2010, Increasing the sensitivity of controlled source electromagnetics by using synthetic aperture, 80th annual SEG meeting, Denver, 805-810. 
- Wapenaar, K., van der Neut, J., Ruigrok, E., Draganov, D., Hunziker, J., Slob, E., Thorbecke, J., and Snieder, R., 2010, Seismic interferometry by crosscorrelation and by multi-dimensional deconvolution: a systematic comparison, American Geophysical Union, 2010 Fall Meeting, San Fransisco.

- Wapenaar, K., van der Neut, J., Ruigrok, E., Draganov, D., Hunziker, J., Slob, E., Thorbecke, J., and Snieder, R., 2010, Seismic interferometry by crosscorrelation and by multidimensional deconvolution: a systematic comparison, EGU, General Assembly 2010, Vienna.

- van der Neut, J., Ruigrok, E., Draganov, D., Thorbecke, J., Hunziker, J., and Wapenaar, K., 2010, Surface wave retrieval from ambient noise using multi-dimensional deconvolution, EAGE Saint Petersburg 2010.

- Minato, S., Matsuoka, T., Tsuji, T., Draganov, D., Hunziker, J., and Wapenaar, K., 2009, Application of seismic interferometry by multidimensional deconvolution to crosswell seismic reflection using singularvalue decomposition, 79th annual SEG meeting, Houston, 1688-1692.

- Slob, E. C., Hunziker, J. and Kuroda S., 2009, GPR imaging strategies for subsurface object detection from coherent radiometry data (invited). In Sato, M (Ed.), Space, Aeronautical and Navigational Electronics, Technical Report, Vol. 109, No. 219 (pp. 55-60). Tokyo, Japan: IEICE.

- van der Neut, J., Hunziker, J., Wapenaar, K., and Slob, E., 2008, Controlled-source seismic interferometry by multi-dimensional deconvolution: stability aspects with various numbers of sources and receivers, 78th annual SEG meeting, Las Vegas, 3008-3012.

- Wapenaar, K., van der Neut, J., Ruigrok, E., Draganov, D., Hunziker, J., Slob, E., Thorbecke, J., and Snieder, R., 2008, Seismic interferometry by multidimensional deconvolution as a means to compensate for anisotropic illumination (invited), American Geophysical Union, 2008 Fall Meeting, San Francisco. 